\title{
Data Repositorv.
}

The data repository contains text detailing the detailed methods of argon isotope and major element data collection of biotite from the RLS. The repository also contains text detailing the numerical modelling of heat-loss from the RLS. Figures DR1-DR5 consist of all the normal and inverse isochron diagrams for each sample, a plateau diagram for each sample, an example of a backscatter image of interlayered chlorite within biotite, and an inverse isochron of previously published biotite Ar-Ar data from the RLS. Also included are summary tables DR1-DR4 including age summaries, electron microprobe results, and tabulated results for all steps for each Ar isotope measured.

\section{$\underline{\text { Data Repository Item DR1 }}$}

\section{Argon Isotope Measurements}

For argon isotopic analyses, biotite separates along with the reference standard, Fish Canyon (FC) sanidine [28.201 Ma; Kuiper et al. (2008)] were placed in Al-disks for neutron irradiation, which was carried out at the US Geological Survey TRIGA reactor for 80 hours without Cd-shielding. At Rutgers University, samples were step-heated using a New Wave 40watt $\mathrm{CO}_{2}$ laser and isotopes were measured on a noble gas mass spectrometer made by Mass Analyzer Products (MAP 215-50) and upgraded by Turrin et al. (2010). A typical ten-minute static system 'cold' blank, $\left(10^{-18} \mathrm{~mol}\right)$ is: ${ }^{40} \mathrm{Ar}=420 \pm 13 ;{ }^{39} \mathrm{Ar}=12.0 \pm 3.6 ;{ }^{38} \mathrm{Ar}=1.0 \pm 0.4$; ${ }^{37} \mathrm{Ar}=22.4 \pm 1.4 ;{ }^{36} \mathrm{Ar}=3.3 \pm 0.3$.

Instrumental mass fractionation was monitored by analyzing a known volume of atmospheric Ar after every eighth sample and blank measurement. For the data reduction we assumed an initial value of ${ }^{38} \mathrm{Ar} /{ }^{36} \mathrm{Ar}=0.1885 \pm 0.0003$ (Lee et al., 2006). As mentioned in the main text, we do not assume an initial ${ }^{40} \mathrm{Ar} /{ }^{36} \mathrm{Ar}=298.56 \pm 0.31$ (Lee et al., 2006), but rather use 
the intercept value from inverse isochron correlations to calculate plateau ages. Signals for reactor produced ${ }^{37} \mathrm{Ar}$ and ${ }^{39} \mathrm{Ar}$ were corrected using the decay constants of 0.0198 day $^{-1}$ and $7.07 \times 10^{-6}$ day $^{-1}$, respectively. ${ }^{37}$ Ar was not used because most of it had decayed by the time of measurement. Instead, a fixed ${ }^{37} \mathrm{Ar} /{ }^{39} \mathrm{Ar}$ value of $0.001 \pm 0.001$ was used in age calculations, a value that is supported by the electron microbe data collected on biotite from the RLS (Table DR2). Automated laser heating, gas extraction, mass spectroscopy measurements, and data reduction were implemented using automated software written by A. Deino of the Berkeley Geochronology Center. The age calculations incorporate the decay constants of Steiger and Jäger, (1977) and the following ratios $\left(/ 10^{-4}\right)$, which are necessary for making reactor corrections: $\left[\left({ }^{36} \mathrm{Ar} /{ }^{37} \mathrm{Ar}\right)_{\mathrm{Ca}}=2.75 \pm 0.003\right) ;\left({ }^{39} \mathrm{Ar} /{ }^{37} \mathrm{Ar}\right)_{\mathrm{Ca}}=6.62 \pm 0.01 ;\left({ }^{38} \mathrm{Ar} /{ }^{37} \mathrm{Ar}\right)_{\mathrm{Ca}}=0.210 \pm$ $0.1 ;\left({ }^{40} \mathrm{Ar}_{\mathrm{K}}{ }^{39} \mathrm{Ar}\right)_{\mathrm{K}}=98.1 \pm 0.18 ;\left({ }^{38} \mathrm{Ar}_{\mathrm{K}} /{ }^{39} \mathrm{Ar}\right)_{\mathrm{K}}=13200 \pm 10 ;\left({ }^{37} \mathrm{Ar}_{\mathrm{K}} /{ }^{39} \mathrm{Ar}\right)_{\mathrm{K}}=2.20 \pm 0.007 ;$ $\left.\left({ }^{36} \mathrm{Ar}_{\mathrm{Cl}}{ }^{38} \mathrm{Ar}\right)_{\mathrm{Cl}}=65000 \pm 3000\right]$.

We define an age plateau as a sequence of three or more consecutive steps with apparent ages that agree within the $95 \%$ confidence interval and for which the sum of ${ }^{39} \mathrm{Ar}_{\mathrm{K}}$ released is $50 \%$ or more of the total ${ }^{39} \mathrm{Ar}_{\mathrm{K}}$ released on completion of the heating experiment (Dalrymple and Lanphere, 1969, 1974; Fleck et al., 1977). The plateau ages and their errors are calculated using the variance-weighted mean of the plateau steps (Taylor, 1997).

\section{Choice of Constants}

Some authors have adopted the most recent determinations for the ${ }^{40} \mathrm{~K}$ decay constants and age of the Fish Canyon sanidine (Renne et al. 2011) in the calculation of ${ }^{40} \mathrm{Ar} /{ }^{39} \mathrm{Ar}$ ages. The values of Renne et al. (2011) can be used with the current dataset by recalculating the ${ }^{40} \mathrm{Ar} /{ }^{39} \mathrm{Ar}$ ages using the equation the following equation from Mercer and Hodges (2016): 


$$
t=\frac{1}{\lambda} \log _{e}\left[\frac{\exp \left[\lambda t_{m}\right]-1}{\exp \left[\lambda_{o} t_{m_{o}}\right]-1}\left(\exp \left[\lambda_{o} t_{o}\right]-1\right)+1\right]
$$

where, $t$ is the new age of measured sample, $t_{m}$ is the new age of the monitor mineral, $t_{m_{o}}$ is the original age of the monitor mineral, $t_{o}$ is the original age of the measured sample, $\lambda$ is the new decay constant, and $\lambda_{o}$ is the original decay constant. When our data is recalculated using the values of Renne et al. (2011), the weighted mean plateau age for all biotite increases from 2054.51 Ma to an implausible age of 2060.40 Ma, older than the U-Pb zircon ages. Similarly, if the Min et al. (2000) decay constants are used (with the Kuiper et al., 2008 value for FC), the weighted mean plateau age for all biotite increases from 2054.51 Ma to an even more implausible age of 2066.26 Ma.

There is currently no consensus within the Ar geochronology community on the "correct" ${ }^{40} \mathrm{~K}$ decay constants to use (e.g. Steiger \& Jäger (1977); Min et al. (2000); Kwon et al. (2002); Renne et al. (2010); Schwarz et al. (2011); Renne et al. (2011). With the rapid changes in proposed decay constants (e.g. Min et al., 2000; Kwon et al., 2002; Renne et al., 2010; Renne et al., 2011), we are of the opinion that until a value is agree upon by the international community, new values should not be used in order to limit confusion and provide consistency. As such, we use the decay constants adopted by the IUGS Subcommission on Geochronology (Steiger and Jäger, 1977) and the reported age (28.201 Ma) of Fish Canyon sanidine of Kuiper et al. (2008). Further justification for this choice is detailed in the section below.

As a secondary standard, the Hb3gr hornblende was irradiated alongside the samples and treated as an unknown. The runs of $\mathrm{Hb} 3 \mathrm{gr}$ result in a weighted mean age of $1082.9 \pm 3.6 \mathrm{Ma}$ $(1 \sigma)$, well within the error limits of published values for Hb3gr, when adjusted for our age of FC 
sanidine [1080 $\pm 4 \mathrm{Ma}$ (Schwarz and Trieloff, 2007); $1079 \pm 8$ (Jourdan et al., 2006)]. The runs of $\mathrm{Hb} 3 \mathrm{gr}$ result in a weighted mean $R_{\mathrm{FCs}}{ }^{\mathrm{Hb} 3 \mathrm{gr}}$ value of $52.24 \pm 0.17(1 \sigma)$.

We have also added a $R_{i}$ value for each age for the reader to readily recalculate these ages, without the use of the Mercer and Hodges (2016) software, using the following equation (Renne at al. 1998), if necessary (Table DR1):

$$
t_{s t d}=\frac{1}{\lambda_{t o t}} \ln \left[\frac{\left(e^{\lambda_{t o t} t_{i-1}}\right)}{R_{i}}+1\right]
$$

where $t_{s t d}$ is the age of the standard, $\lambda_{\text {tot }}$ is the total ${ }^{40} \mathrm{~K}$ decay constant and $t_{i}$ is the new age of the sample.

\section{Errors From ${ }^{40} \mathrm{~K}$ Decay Constant}

As mentioned in the main text the weighted mean of all ${ }^{40} \mathrm{Ar} /{ }^{39} \mathrm{Ar}$ biotite ages increases from $2054.47 \pm 0.84 \mathrm{Ma}$ to $\pm 21 \mathrm{Ma}$ when all external uncertainties are accounted [(e.g. ${ }^{40} \mathrm{~K}$ decay constants]), and allows for many thermal models to fit (including the conductive cooling model). While we acknowledge this external uncertainty, we base our interpretations on the measured ages and their internal uncertainties. Our justification is that our ${ }^{40} \mathrm{Ar} /{ }^{39} \mathrm{Ar}$ ages are directly tied to the U-Pb system by two standards that were co-irradiated with our samples, Fish Canyon at 28.2 Ma and $\mathrm{Hb} 3 \mathrm{gr}$ at $1082 \mathrm{Ma}$.

The chosen age (as described above) of 28.201 Ma (Kuiper et al. 2008) for the Fish Canyon Sanidine matches closely with the "28.2 Ma" age from the younger mode of U-Pb zircons for the Fish Canyon unit (Wotzlaw et al. 2013). The U-Pb measurements for this "young" mode indicate an age of $28.206 \pm 0.022 \mathrm{Ma}$ (Wotzlaw et al. 2013), an extremely close match to the Astronomically calibrated value (28.201 Ma) of Kuiper et al., 2008. 
The $\mathrm{Hb} 3 \mathrm{gr}{ }^{40} \mathrm{Ar} /{ }^{39} \mathrm{Ar}$ standard, described above, is an amphibole from the Lone Grove Pluton that we routinely co-irradiate with FC (28.201 Ma) when measuring Archean samples. Blackburn et al. (2017) report a U-Pb zircon crystallization age of $1090.10 \pm 0.16 \mathrm{Ma}$ and a youngest titanite cooling age of $1082.2 \pm 0.75 \mathrm{Ma}$ for the Lone Grove pluton (Note: the U-Pb crystallization temperature of zircons and closure temperature of titanite is $\sim 900^{\circ} \mathrm{C}$ and $\sim 600^{\circ} \mathrm{C}$, respectively). Titanite ages from Blackburn et al. (2017) within error of this youngest age, indicate a mean "young" titanite age of $1082.9 \pm 0.58 \mathrm{Ma}$. The ${ }^{40} \mathrm{Ar} /{ }^{39} \mathrm{Ar}$ closure temperature of amphibole $\left(\sim 500-600^{\circ} \mathrm{C}\right.$; Harrison, 1982 ; McDougall and Harrison, 1999$)$ is similar to the U-Pb closure temperature of titanite, and thus the ages should be similar.

As mentioned above, we routinely co-irradiate Hb3gr with FC (28.201 Ma) when measuring Archean samples. The co-irradiated $\mathrm{Hb} 3 \mathrm{gr}$ from this study result in a result in a weighted mean ${ }^{40} \mathrm{Ar} /{ }^{39} \mathrm{Ar}$ age of $1082.9 \pm 3.6 \mathrm{Ma}(1 \sigma)$.

Now, we re-introduce the R-notation (as in Renne et al., 2010):

$$
R \frac{H B}{F C} \equiv \frac{\left(e^{\lambda_{t o t} t_{H B-1}}\right)}{\left(e^{\left.\lambda_{t o t} t_{F C}-1\right)}\right.} \equiv \frac{\left(\frac{{ }^{40} A r^{*}}{{ }^{39} A r_{K}}\right)_{(H B)_{m e a s}}}{\left(\frac{{ }^{40} A r^{*}}{{ }^{39} A r_{K}}\right)_{(F C)_{m e a s .}}}
$$

where $\lambda_{\text {tot }}$ is the chosen total ${ }^{40} \mathrm{~K}$ decay constant, and $t_{F C}$ and $t_{H B}$ is are the U-Pb ages of the standards (in this case FC and $\mathrm{Hb} 3 \mathrm{gr}$, respectively).

By varying the chosen decay constants, an $R_{\mathrm{FCs}}{ }^{\mathrm{Hb} 3 g r}$ value can be calculated using the $\mathrm{U}$ $\mathrm{Pb}$ values from the same units, namely 28.206 Ma for Fish Canyon (mean of younger mode; Wotzlaw et al., 2013) and 1082.9 Ma for Hb3gr (mean young titanite ages; Blackburn et al., 2017). Comparing the "U-Pb" calibrated $R_{\mathrm{FCs}}{ }^{\mathrm{Hb} 3 \mathrm{gr}}$ values shows that our measured $R_{\mathrm{FCs}}{ }^{\mathrm{Hb} 3 \mathrm{gr}}$ of 
52.24 best matches the "U-Pb" calibrated $R_{\mathrm{FCs}}{ }^{\mathrm{Hb} 3 g r}$ using Steiger and Jäger (1977) $\left[R_{\mathrm{FCs}}{ }^{\mathrm{Hb} 3 g r}=\right.$ 52.21], with only a 0.6 per-mill difference. This is compared to the "U-Pb" calibrated $R_{\mathrm{FCs}}{ }^{\mathrm{Hb} 3 g r}$ using the decay constants of Min et al. $(2000)\left[R_{\mathrm{FCs}}{ }^{\mathrm{Hb} 3 g r}=51.97\right]$ which results in a 5 per-mill

difference. We also note, that the reported $R_{\mathrm{FCs}}{ }^{\mathrm{Hb} 3 \mathrm{gr}}$ of $51.88 \pm 0.06(1 \sigma)$ of Renne et al. (2011), using their preferred standard ages, also results in a 5 per-mill difference.

In summary, using the U-Pb age of FC [28.206 Ma, indistinguishable from 28.201 (Kuiper et al. 2008)] brings us within the EARTHTIME goal of 1-2 per-mill agreement of the U$\mathrm{Pb}$ and ${ }^{40} \mathrm{Ar} /{ }^{39} \mathrm{Ar}$ chronometers. Following this line of reasoning, we are justified in using measurement errors in our interpretation of the thermal model.

\section{Discordant Age Spectra - Chloritization}

In the ${ }^{40} \mathrm{Ar} /{ }^{39} \mathrm{Ar}$ dating of biotite from the UG-2 chromitite layer in the upper Critical Zone, Nomade et al. (2004) noted that $\sim 50 \%$ of individual biotite grains yielded concordant age spectra, while the rest yielded strongly discordant age spectra. Strongly discordant age spectra are characterized by young apparent ages in early heating steps, followed by anomalously old ages due to the various amount of interlayered chlorite within the grains. During irradiation, ${ }^{39} \mathrm{Ar}$ is recoiled into interlayered $\mathrm{K}_{2} \mathrm{O}$-poor chlorite from $\mathrm{K}_{2} \mathrm{O}$-rich biotite and results in low apparent ages in early heating steps (Hess et al., 1987; Min et al., 2001; Nomade et al., 2004). As stepheating progresses, biotite (now depleted in ${ }^{39} \mathrm{Ar}$ ) becomes the main Ar releasing reservoir, resulting in anomalously old apparent ages.

Eight individual biotite grains from the Main Zone in this study also exhibit discordance accompanied by anomalously old ages (as calculated when using an initial ${ }^{40} \mathrm{Ar} /{ }^{36} \mathrm{Ar}=298.56 \pm$ 0.31), consistent with the interlayered chlorite found within these samples (Fig. DR4). After concluding little to no Ar-loss from the UG-2 biotite, Nomade et al. (2004) use the total fusion 
age of each discordant sample as a comparison to the plateau ages yielded from concordant age spectra. Some samples in our study, however, show evidence of Ar-loss from a reheating event and we therefore cannot use the total fusion age as a direct comparison to other individual analyses. Instead, we use the isochron and inverse isochron regressions to deduce an age, trapped component and finally, a re-calculated plateau age for these discordant samples.

For the eight discordant biotite samples, isochron and inverse isochron relationships for heating-steps dominated by Ar release from biotite yield ages similar to the plateau ages of concordant analyses $(\sim 2055 \mathrm{Ma})$ but also indicate excess ${ }^{40} \mathrm{Ar}$. Individual age spectra can be corrected for this trapped component by re-calculating the age spectra using the ${ }^{40} \mathrm{Ar} /{ }^{36} \mathrm{Ar}$ intercept value from an inverse isochron regression. This results in the elimination the chloritedominated portion of the age spectra (low apparent ages at early gas release steps) and results in a plateau age for the biotite dominated portion of gas release that is indistinguishable from the isochron ages (Fig. DR3). No isochrons were originally reported in the study of Nomade et al. (2004), however despite low precision in ${ }^{36} \mathrm{Ar}$ measurements, a similar general relationship can be found by re-analyzing the data of Nomade et al. (2004) (Fig. DR5).

\section{Data Repository Item DR2}

\section{Thermal Modelling}

Previous thermal modelling of the Bushveld Complex by Zeh et al. (2015), which produced a cooling model that matched observed $\mathrm{U}-\mathrm{Pb}$ zircon ages, assumed no additional heat was produced (e.g. radiogenic or latent heat), that no advection took place and that the magma body was an infinite sheet. The assumption of in an infinite sheet (a 1-D model) was also made by Cawthorn and Webb (2013) and allows for heat loss only through the top and bottom of the intrusion, with no heat loss through the sides. With these assumptions in place, the cooling 
profile for the magma body can be solved using the following heat flow equation (Carslaw and Jaeger, 1959):

$\frac{T-T_{S}}{T_{o}-T_{S}}=\frac{1}{2}\left(\operatorname{erf}\left(\frac{a-x}{2 \sqrt{k t}}\right)+\operatorname{erf}\left(\frac{a+x}{2 \sqrt{k t}}\right)\right)$

Where:

$\mathrm{T}=$ actual temperature at position $\mathrm{x}\left({ }^{\circ} \mathrm{C}\right)$

$\mathrm{T}_{\mathrm{s}}=$ initial temperature of host $\operatorname{rock}\left({ }^{\circ} \mathrm{C}\right)$

$\mathrm{T}_{\mathrm{o}}=$ initial temperature of the magma body $\left({ }^{\circ} \mathrm{C}\right)$

$\mathrm{a}=$ half thickness of the magma body $(\mathrm{m})$

$\mathrm{x}=$ distance from center of magma body $(\mathrm{m})$

$\mathrm{k}=$ thermal conductivity $\left(\mathrm{m}^{2} / \mathrm{s}\right)$

$\mathrm{t}=$ time $(\mathrm{s})$

Also, where the initial temperature of the host rock $\left(T_{s}\right)$ is calculated for a given depth within the intrusion assuming an elevated local geothermal gradient of $30^{\circ} \mathrm{C} \mathrm{km}^{-1}$ (Gibson \& Wallmach, 1995) and an average surface temperature of $15^{\circ} \mathrm{C}$.

Whereas the samples from this study do not come from the exact center of an infinite sheet, but rather from closer to the contact with the host rocks, we also solve the heat flow equation while allowing heat loss through the sides of the intrusion (a 2-D model). This cooling profile can be solved using a modification of equation (4) of Carslaw and Jaeger (1959):

$\frac{T-T_{s}}{T_{0}-T_{s}}=\left[\frac{1}{2}\left(\operatorname{erf}\left(\frac{a-x}{2 \sqrt{k t}}\right)+\operatorname{erf}\left(\frac{a+x}{2 \sqrt{k t}}\right)\right)\right]\left[\frac{1}{2}\left(\operatorname{erf}\left(\frac{b-y}{2 \sqrt{k t}}\right)+\operatorname{erf}\left(\frac{b+y}{2 \sqrt{k t}}\right)\right)\right]\left[\frac{1}{2}\left(\operatorname{erf}\left(\frac{c-z}{2 \sqrt{k t}}\right)+\operatorname{erf}\left(\frac{c+z}{2 \sqrt{k t}}\right)\right)\right]$

Where the following new variables are introduced:

$\mathrm{b}=$ half thickness of the magma body in horizontal direction "b" (i.e. East-West)

$\mathrm{y}=$ distance from center of magma body ( $\mathrm{b}$ - direction)

$\mathrm{c}=$ half thickness of the magma body in horizontal direction "c" (i.e. North-South)

$\mathrm{z}=$ distance from center of magma body $(\mathrm{c}-$ direction)

The cooling models are solved using an initial temperature $\left(T_{0}\right)$ of $1250^{\circ} \mathrm{C}$ for an $8 \mathrm{~km}$ sheet of magma. The choice of initial temperature has little effect on the final cooling model, as the 
model is built to pass through the observed $\mathrm{U}-\mathrm{Pb}$ zircon ages and estimated temperatures. Both models are solved for a point at the center of the intrusion (i.e. $4 \mathrm{~km}$ from the roof contact), with an estimated $4 \mathrm{~km}$ of roof rocks on top of the intrusion (in terms of depth). A $4 \mathrm{~km}$ thick roof is consistent with the roof being composed of $2 \mathrm{~km}$ Rooiberg Group Lavas (Schweitzer et al., 1997) with $2 \mathrm{~km}$ of the coeval and related Bushveld Granite on top. In the case of the 2-D model, the point being solved for is still located at the center of the intrusion in terms of depth $(\mathrm{x}=0 \mathrm{~m})$, as well as at the center of the intrusion in terms of its North-South dimension $(\mathrm{z}=0 \mathrm{~m})$.

However, the point is now placed at $15 \mathrm{~km}$ from the Eastern edge of the Bushveld Complex, while assuming a $450 \mathrm{~km}$-wide intrusion $(\mathrm{b}=225,000 \mathrm{~m} ; \mathrm{y}=210,000 \mathrm{~m})$. This placement allows for a significant amount of heat loss through the sides of the intrusion and provides a likely more realistic estimate of the cooling profile of the solidified body.

It is important to note that the Bushveld Granite is not being modelled as a later magma in this scenario, but rather as part of the roof with the temperature set by the geothermal gradient. The choice (similar to that of Zeh et al., 2015) implies that these models display the fastest possible cooling of the mafic body, as a later or even exactly coeval emplacement would result in additional heat being provided to the RLS and the surrounding rocks. As the observed data already do not fit the conductive cooling model without the additional heat from the Bushveld Granite, the same conclusion would be reached if it were included.

\section{Data Repository Item DR3}

\section{Electron Microprobe}

In situ major element compositions for biotite were determined using the JEOL JXA 8200 electron microprobe at Rutgers University. All analyses used an acceleration potential of 15 
$\mathrm{kV}$, beam current of $15 \mathrm{nA}$, and a beam diameter of $5 \mu \mathrm{m}$. Count times consisted of $15 \mathrm{~s}$ on background for each element, while peak count times and primary standard varied per element ( $\mathrm{Si}$ - Biotite Kristiansand $=20 \mathrm{~s}, \mathrm{Mg}$ - F-phlogopite stoichiometric $=20 \mathrm{~s}, \mathrm{Al}$ - Biotite Kristiansand $=20 \mathrm{~s}, \mathrm{Na}-$ Jadeite $=10 \mathrm{~s}, \mathrm{~F}-\mathrm{F}$-phlogopite stoichiometric $=10 \mathrm{~s}, \mathrm{Fe}-$ Biotite Kristiansand $=10 \mathrm{~s}, \mathrm{Mn}-$ Rhodonite $=40 \mathrm{~s}, \mathrm{Ba}-$ Benitoite $=45 \mathrm{~s}, \mathrm{Cl}-\mathrm{Cl}$-Apatite Kragero $=10$ s, $\mathrm{Cr}-\mathrm{Mg}_{2} \mathrm{CrO}_{4}$ chromite $=40 \mathrm{~s}, \mathrm{Ca}-$ Apatite Wilberforce $=40 \mathrm{~s}, \mathrm{~K}-\mathrm{F}-$ phlogopite stoichiometric $=10 \mathrm{~s}, \mathrm{Ti}-\mathrm{TiO}_{2}$ Synthetic $\left.=40 \mathrm{~s}\right) . \mathrm{H}_{2} \mathrm{O}$ calculated based on $\mathrm{Cl}+\mathrm{F}+\mathrm{OH}=2.0$ per formula unit.

\section{$\underline{\text { References }}$}

Cawthorn, R. G., and Webb, S. J., 2013, Cooling of the Bushveld Complex, South Africa: implications for paleomagnetic reversals, Geology, v. 41, no. 6, p. 687-690.

Dalrymple, G. B., and Lanphere, M. A., 1969, Potassium-argon dating: Principles, techniques and applications to geochronology: San Francisco, California, Freeman, p. 258-258.

Dalrymple, G. B., and Lamphere, M. A., 1974, 40Ar/39Ar age spectra of some undisturbed terrestrial samples: Geochimica et Cosmochimica Acta, v. 38, no. 5, p. 715-738.

Fleck, R. J., Sutter, J. F., and Elliot, D. H., 1977, Interpretation of discordant 40Ar/39Ar agespectra of Mesozoic tholeiites from Antarctica: Geochimica et Cosmochimica Acta, v. 41, no. 1, p. 15-32.

Harrison, T. M., 1982, Diffusion of $40 \mathrm{Ar}$ in hornblende, Contributions to Mineralogy and Petrology, v. 78, no. 3, p. 324-331.

Hess, J. C., Lippolt, H. J., and Wirth, R., 1987, Interpretation of 40Ar/39Ar biotites: Evidence from hydrothermal degassing experiments and TEM studies: Chemical Geology: Isotope Geoscience section, v. 66, no. 1-2, p. 137-149.

Jourdan, F., Verati, C., and Féraud, G., 2006, Intercalibration of the Hb3gr 40Ar/39Ar dating standard: Chemical Geology, v. 231, no. 3, p. 177-189.

Kwon, J., Min, K., Bickel, P. J., and Renne, P. R., 2002, Statistical methods for jointly estimating the decay constant of $40 \mathrm{~K}$ and the age of a dating standard: Mathematical Geology, v. 34, no. 4, p. 457-474.

Ludwig, K. R., 2003, ISOPLOT a plotting and regression program for radiogenic isotope data, Version Ex./3.00.

Mercer, C. M., and Hodges, K. V., 2016, ArAR - A software tool to promote the robust comparison of $\mathrm{K}-\mathrm{Ar}$ and $40 \mathrm{Ar} / 39 \mathrm{Ar}$ dates published using different decay, isotopic, and monitor-age parameters: Chemical Geology, v. 440, p. 148-163. 
Min, K., Mundil, R., Renne., P. R., and Ludwig, K. R., 2000, A test for systematic errors in 40Ar/39Ar geochronology through comparison with $\mathrm{U} / \mathrm{Pb}$ analysis of a 1.1 Ga rhyolite: Geochimica et Cosmochimica Acta, v. 64, no. 1, p. 73-98.

Min, K., Renne, P. R., and Huff, W. D., 2011, 40Ar/39Ar dating of Ordovician K-bentonites in Laurentia and Baltoscandia: Earth and Planetary Science Letters, v. 185, no. 1-2, p. 121 134.

Renne, P. R., Mundil, R., Balco, G., Min, K., and Ludwig, K. R., 2010, Join determination of $40 \mathrm{~K}$ decay constants and $40 \mathrm{Ar} * 40 \mathrm{~K}$ for the Fish Canyon sanidine standard, and improved accuracy for 40Ar/39Ar geochronology: Geochimica et Cosmochimica Acta, v. 74, no. 18 , p. 5349-5367.

Renne, P. R., Balco, G., Ludwig, K. R., Mundil, R., and Min, K., 2011, Response to thecomment by WH Schwarz et al. on "Joint determination of $40 \mathrm{~K}$ decay constants and $40 \mathrm{Ar} * / 40 \mathrm{~K}$ for the Fish Canyon sanidine standard, and improved accuracy for 40Ar/39Ar geochronology" by PR Renne et al. (2010): Geochimica et Cosmochimica Acta, v. 75, no. 17, p. 5097-5100.

Renne, P. R., Swisher, C. C., Deino, A. L., Karner, D. B., Owens, T. L., and DePaolo, D. J., 1998, Intercalibration of standards, absolute ages and uncertainties in 40Ar/39Ar dating, Chemical Geology, v. 145, no. 1-2, p. 117-152.

Schwarz, W. H., Kossert, K., Trieloff, M., and Hopp, J., 2011, Comment on the "Joint determination of $40 \mathrm{~K}$ decay constants and $40 \mathrm{Ar}^{*} / 40 \mathrm{~K}$ for the Fish Canyon sanidine standard, and improved accuracy for 40Ar/39Ar geochronology" by Paul R. Renne et al. (2010): Geochimica et Cosmochimica Acta, v. 75, no. 17, p. 5094-5096.

Schwarz, W. H., and Trieloff, M., 2007, Intercalibration of 40Ar-39Ar age standards NL-25, HB3gr hornblende, GA1550, SB-3, HD-B1 biotite and BMus/2 muscovite: Chemical Geology, v. 242, no. 1-2, p. 218-231.

Schweitzer, J. K., Hatton, C. J., and De Waal, S. A., 1997, Link between the granitic and volcanic rocks of the Bushveld Complex, South Africa: Journal of African Earth Sciences, v. 24, no. 1-2, 95-104.

Taylor, J., 1997, Introduction to error analysis, the study of uncertainties in physical measurements.

Turrin, B. D., Swisher, C. C., and Deino, A. L., 2010, Mass discrimination monitoring and intercalibration of dual collectors in noble gas mass spectrometer systems: Geochemistry, Geophysics, Geosystems, v. 11, no. 8. 

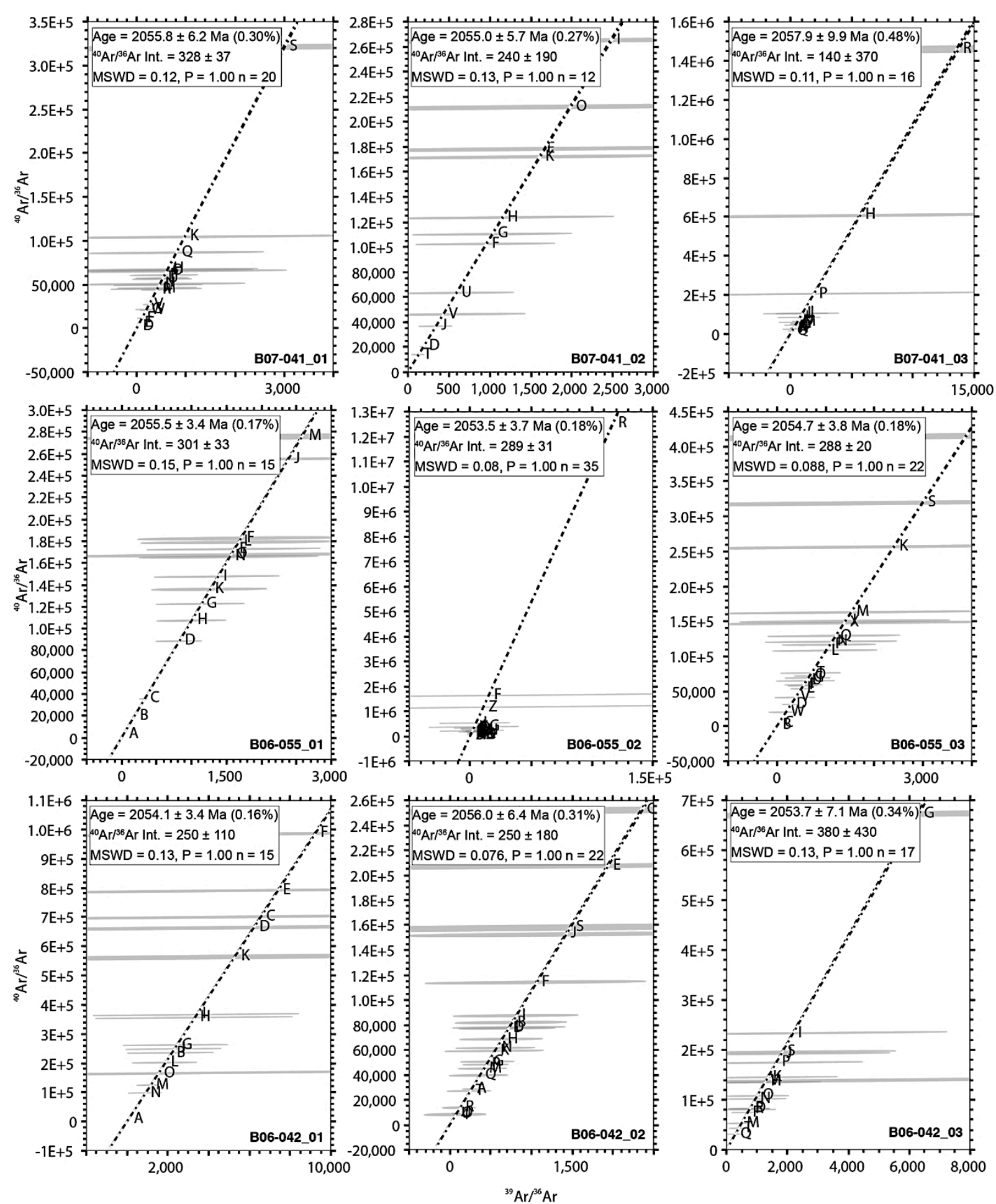

Figure DR1. All Isochrons. Error ellipses, calculated ages, and ${ }^{40} \mathrm{Ar} /{ }^{36} \mathrm{Ar}$ Int. values are displayed with $1 \sigma$ uncertainties. 

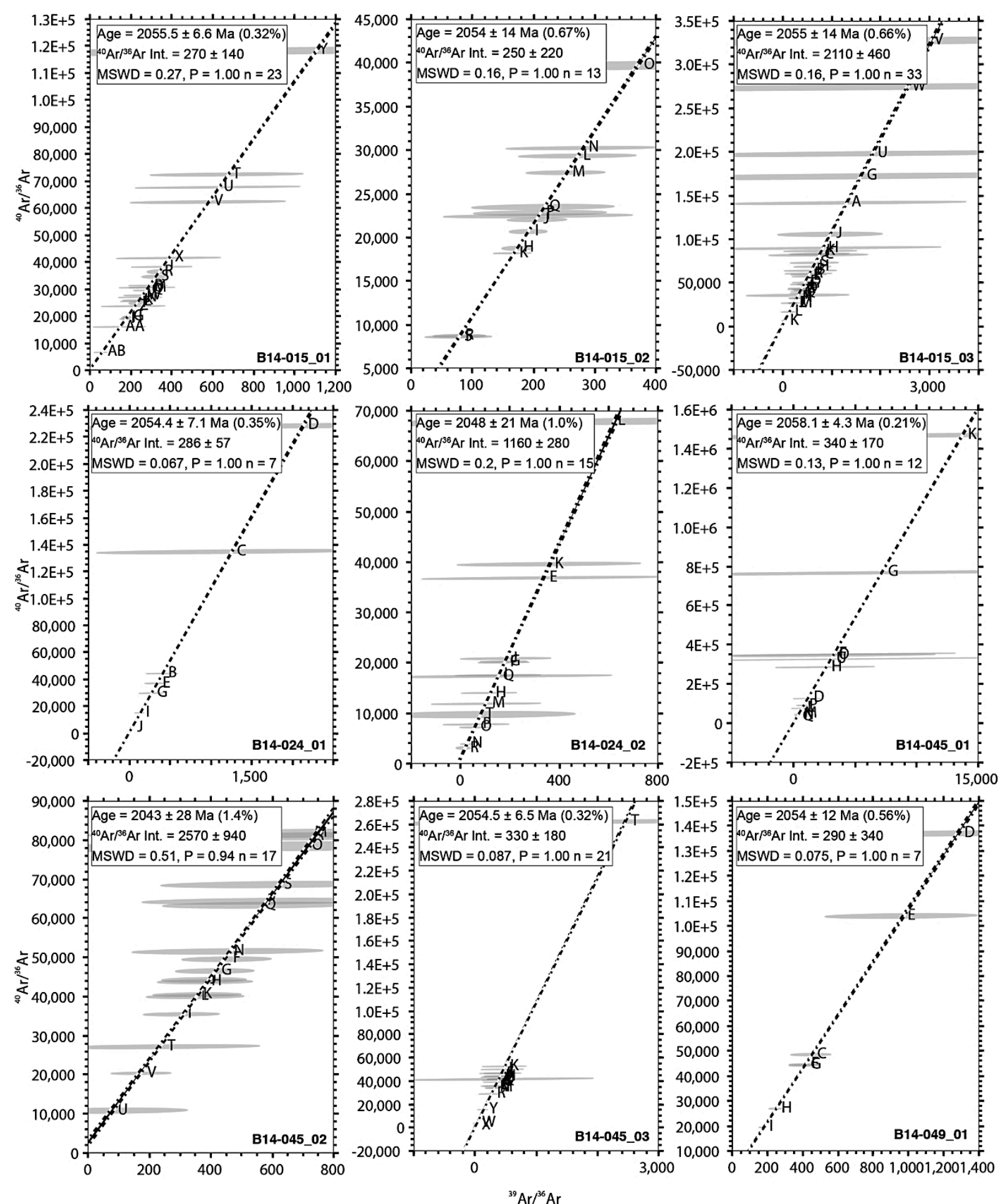

Figure DR1. All Isochrons. (Continued) 


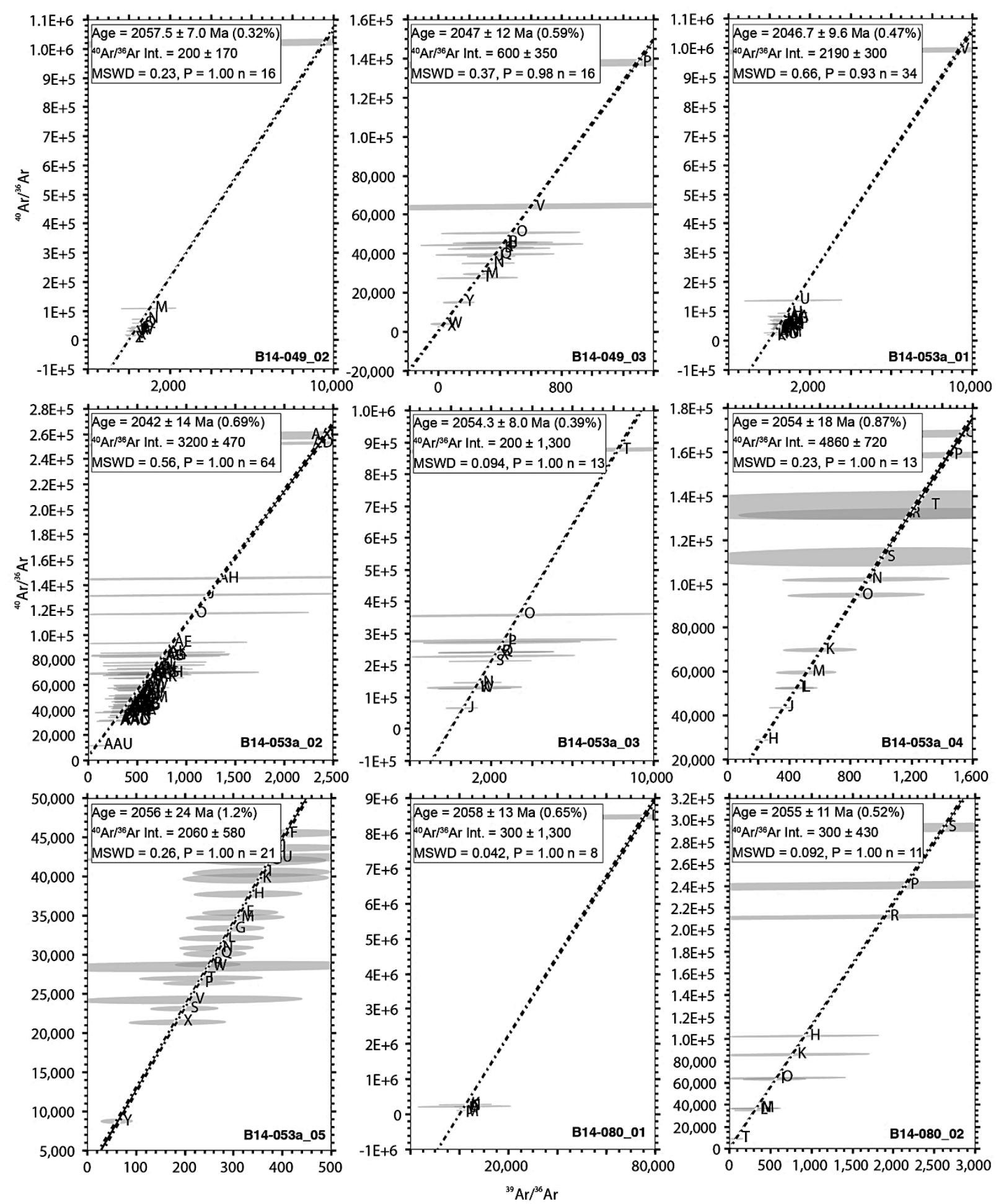

Figure DR1. All Isochrons. (Continued) 

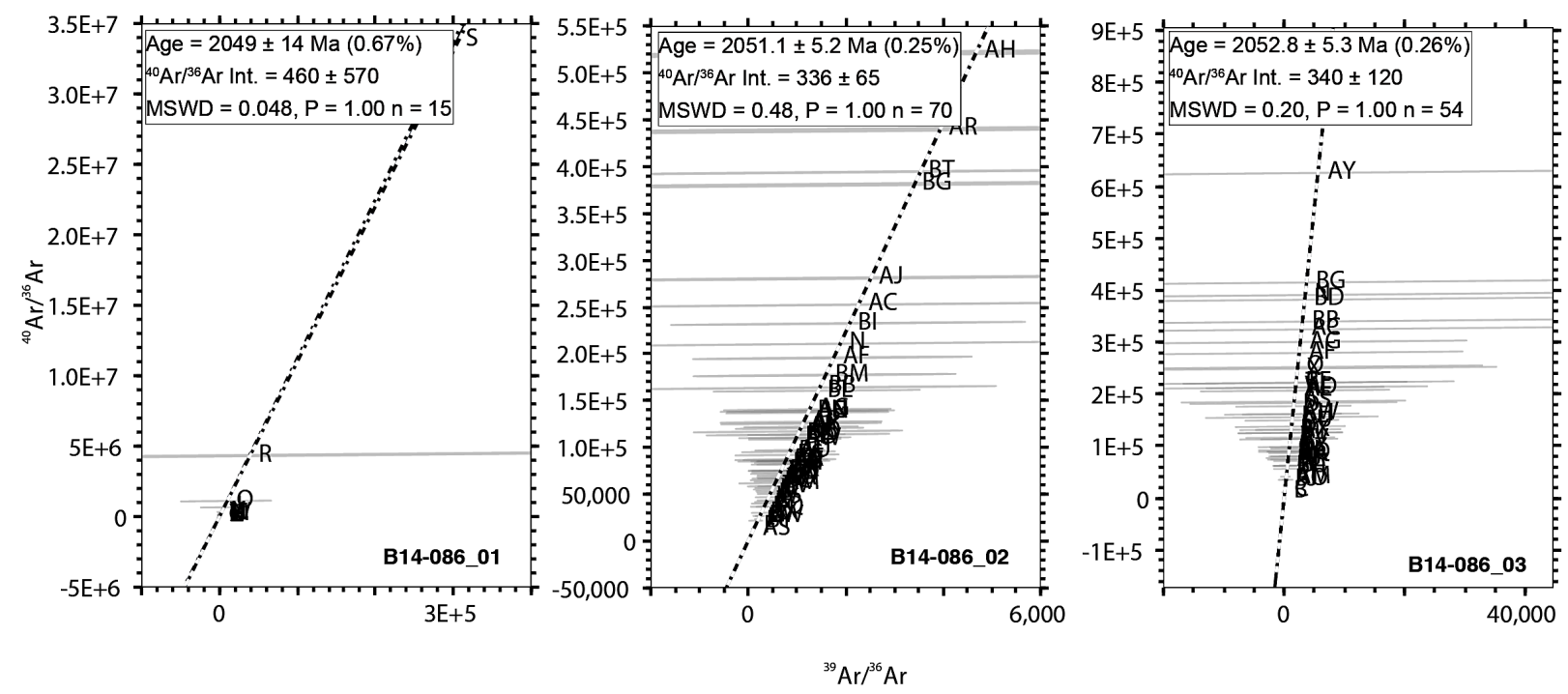

Figure DR1. All Isochrons. (Continued) 

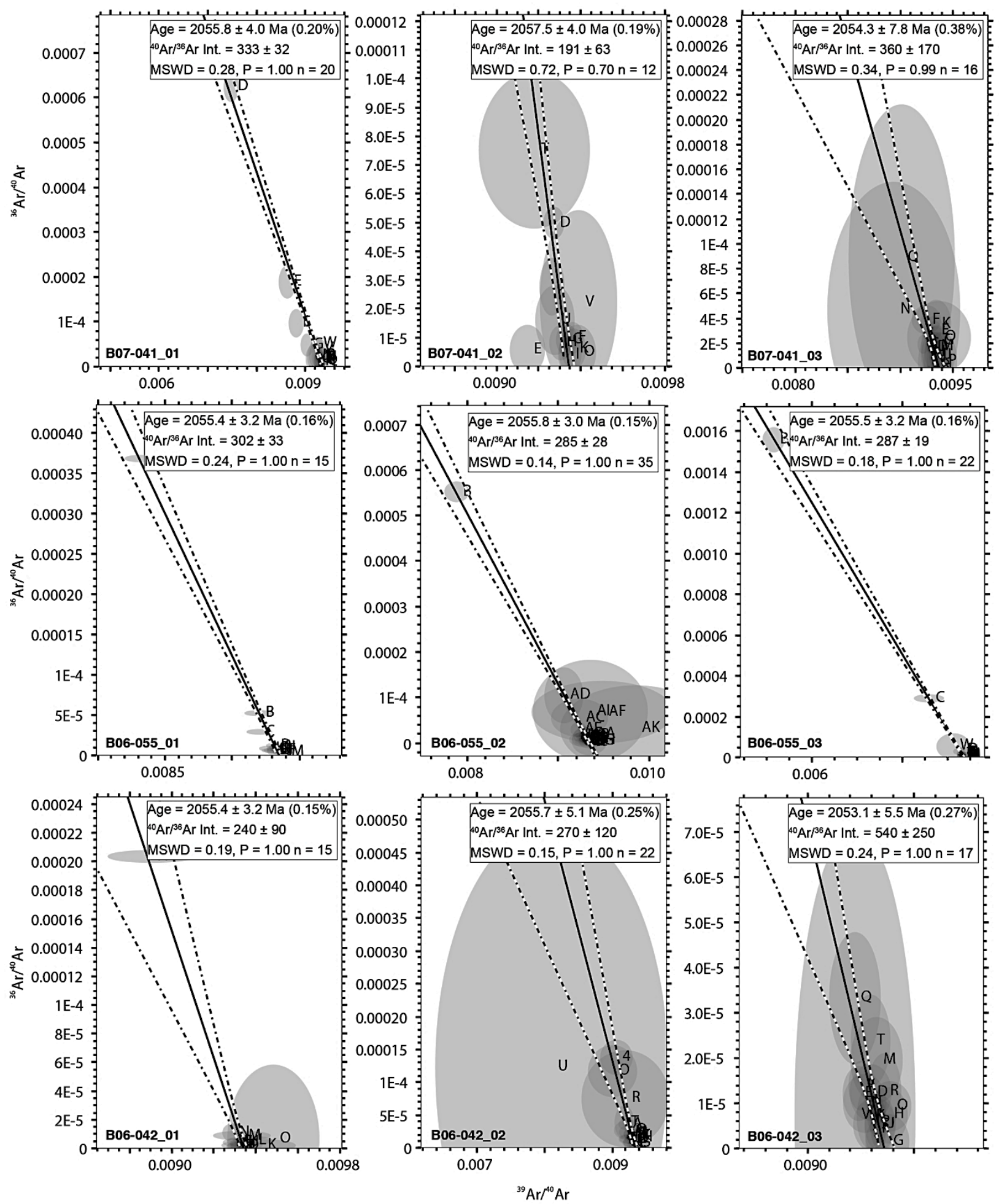

Figure DR2. All Inverse Isochrons. Error ellipses, calculated ages, and ${ }^{40} \mathrm{Ar} /{ }^{36} \mathrm{Ar}$ Int. values are displayed with $1 \sigma$ uncertainties. 

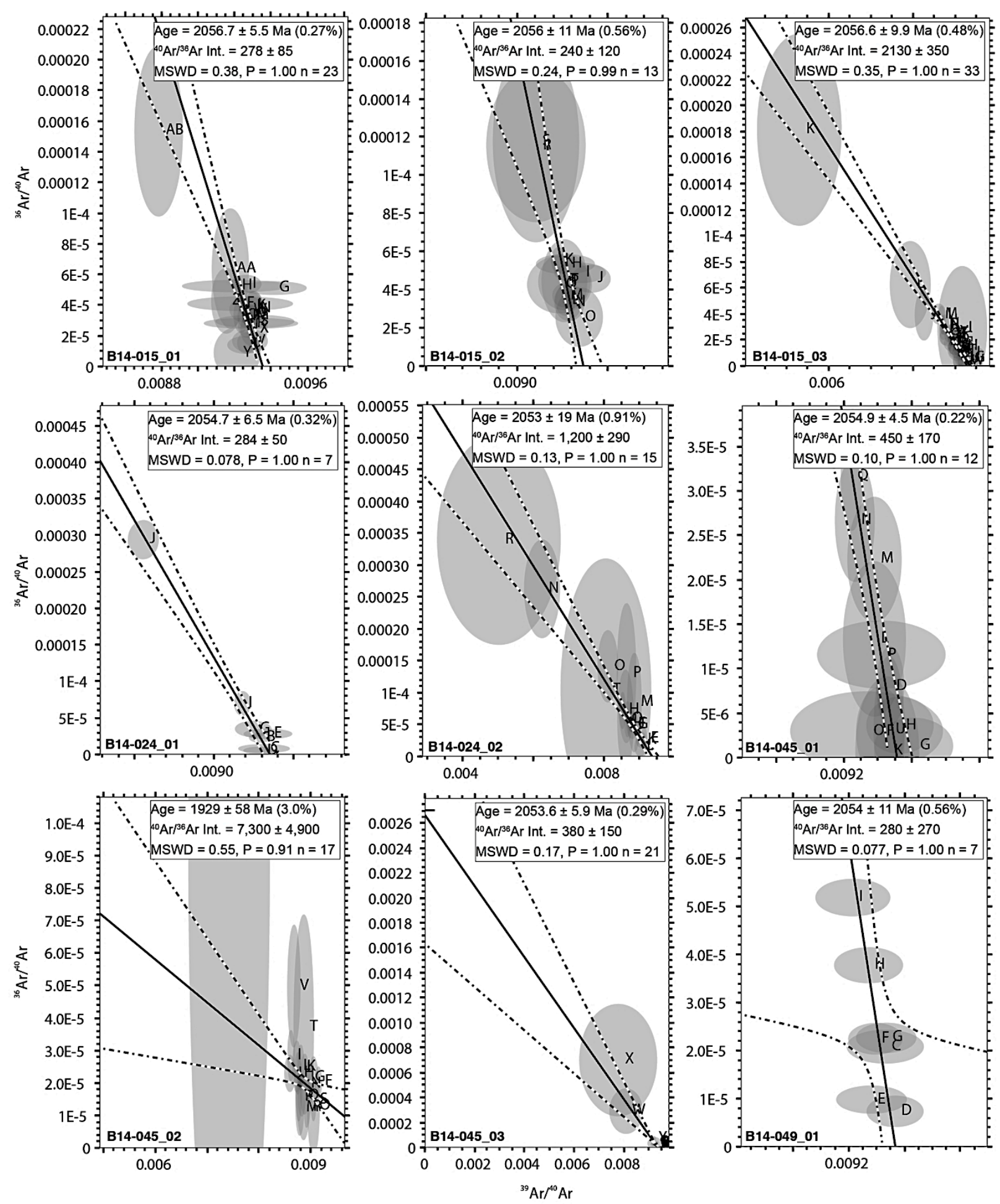

Figure DR2. All Inverse Isochrons. (Continued) 

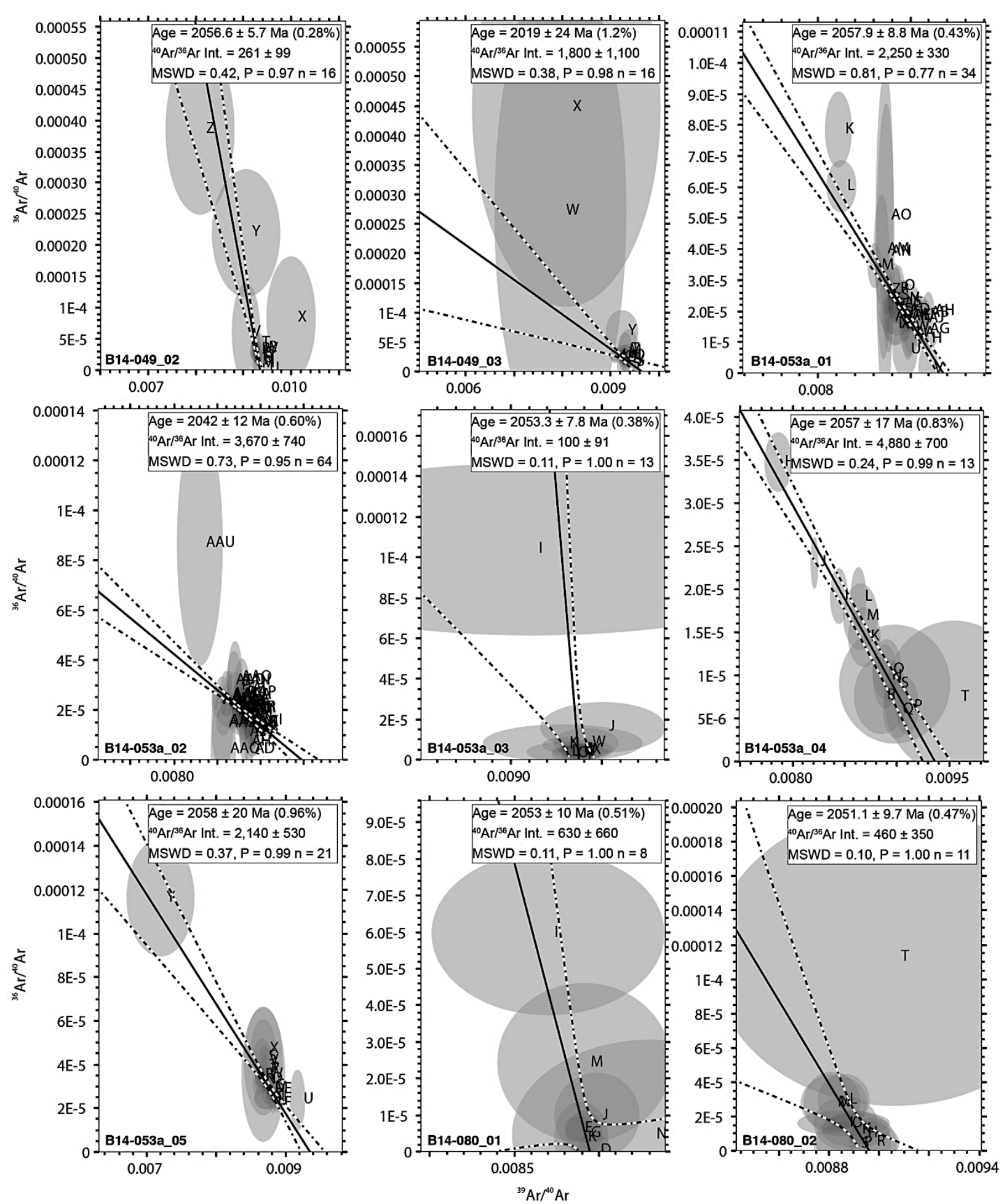

Figure DR2. All Inverse Isochrons. (Continued) 


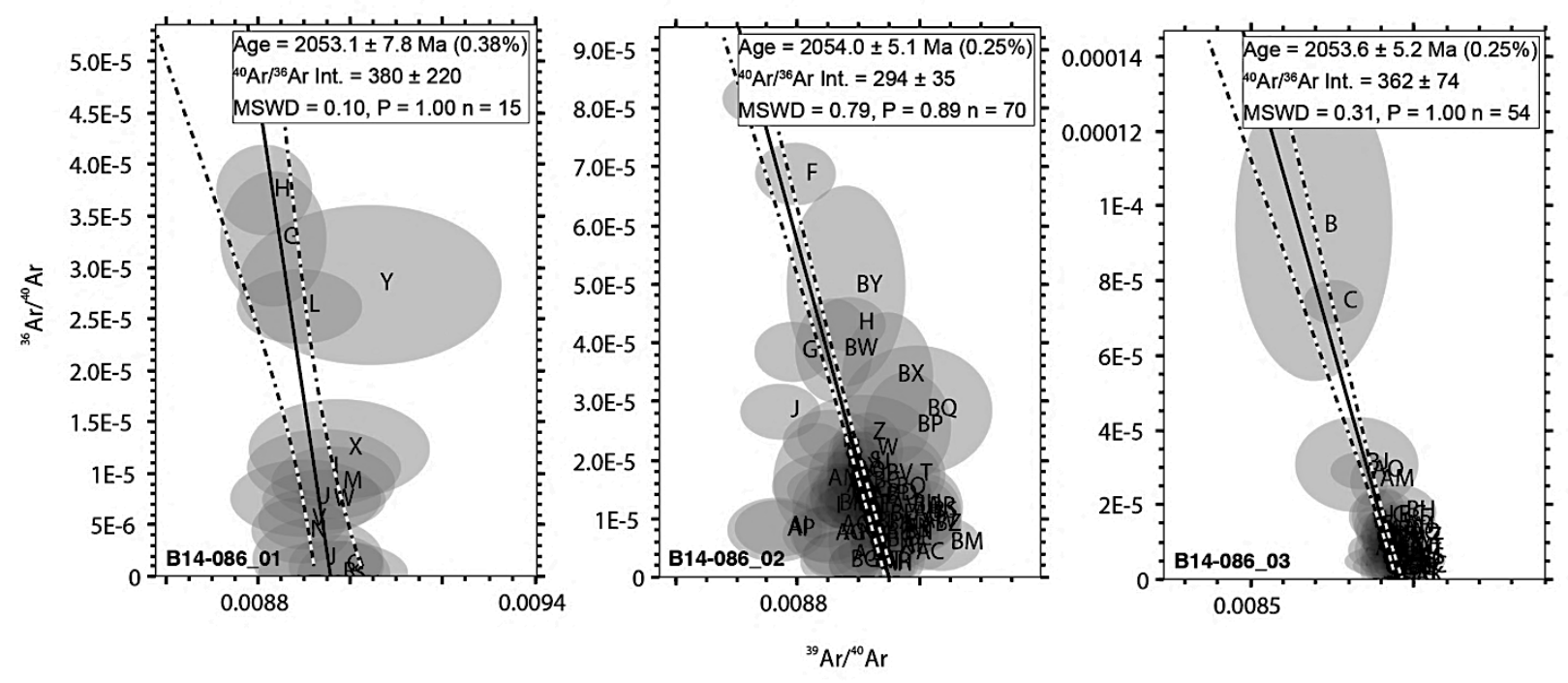

Figure DR2. All Inverse Isochrons. (Continued) 

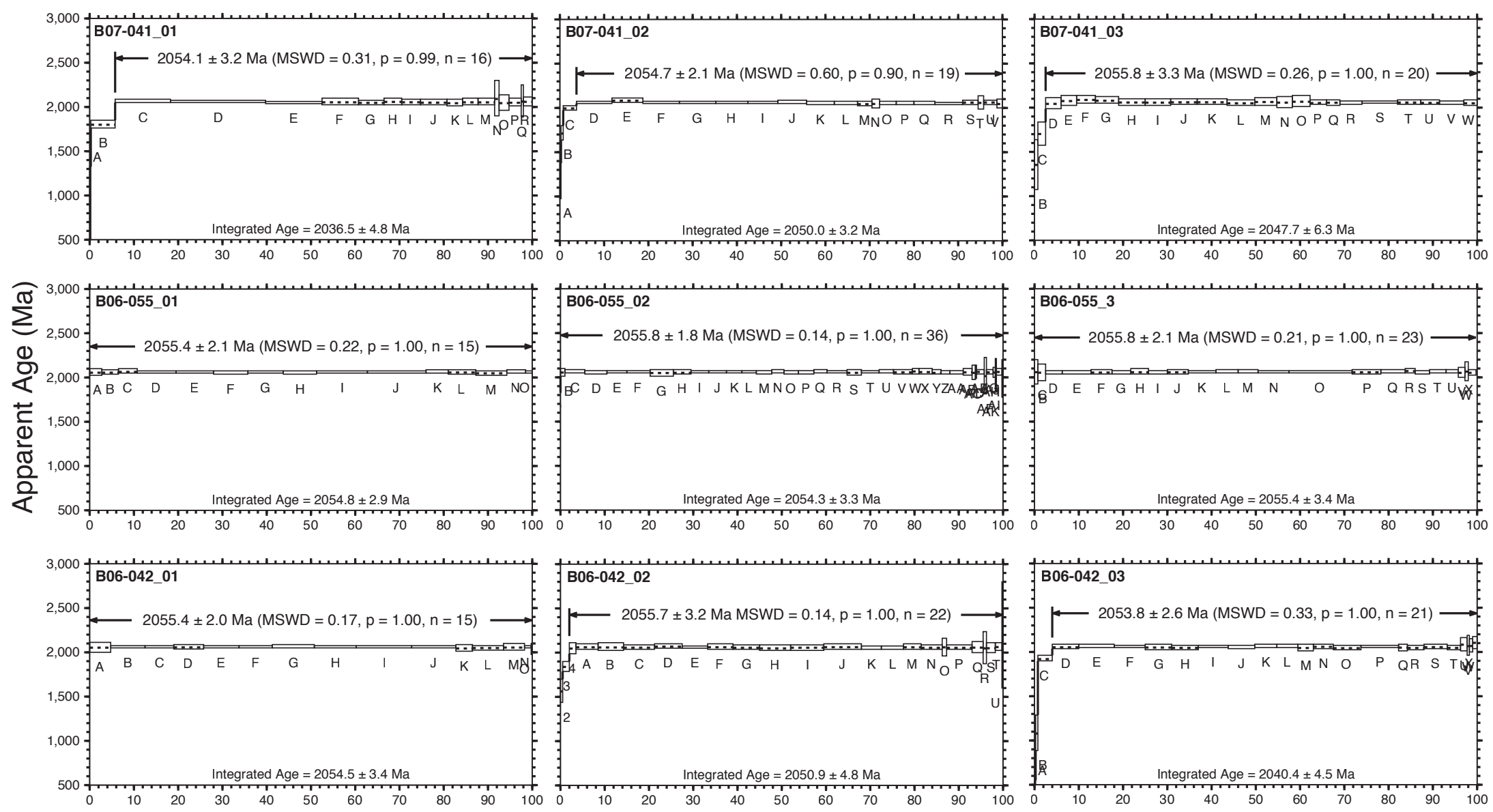

Cumulative $\%{ }^{39} \mathrm{Ar}$ Released

Figure DR3. All Release Spectra. Data and calculated ages are presented with $1 \sigma$ uncertainties. 

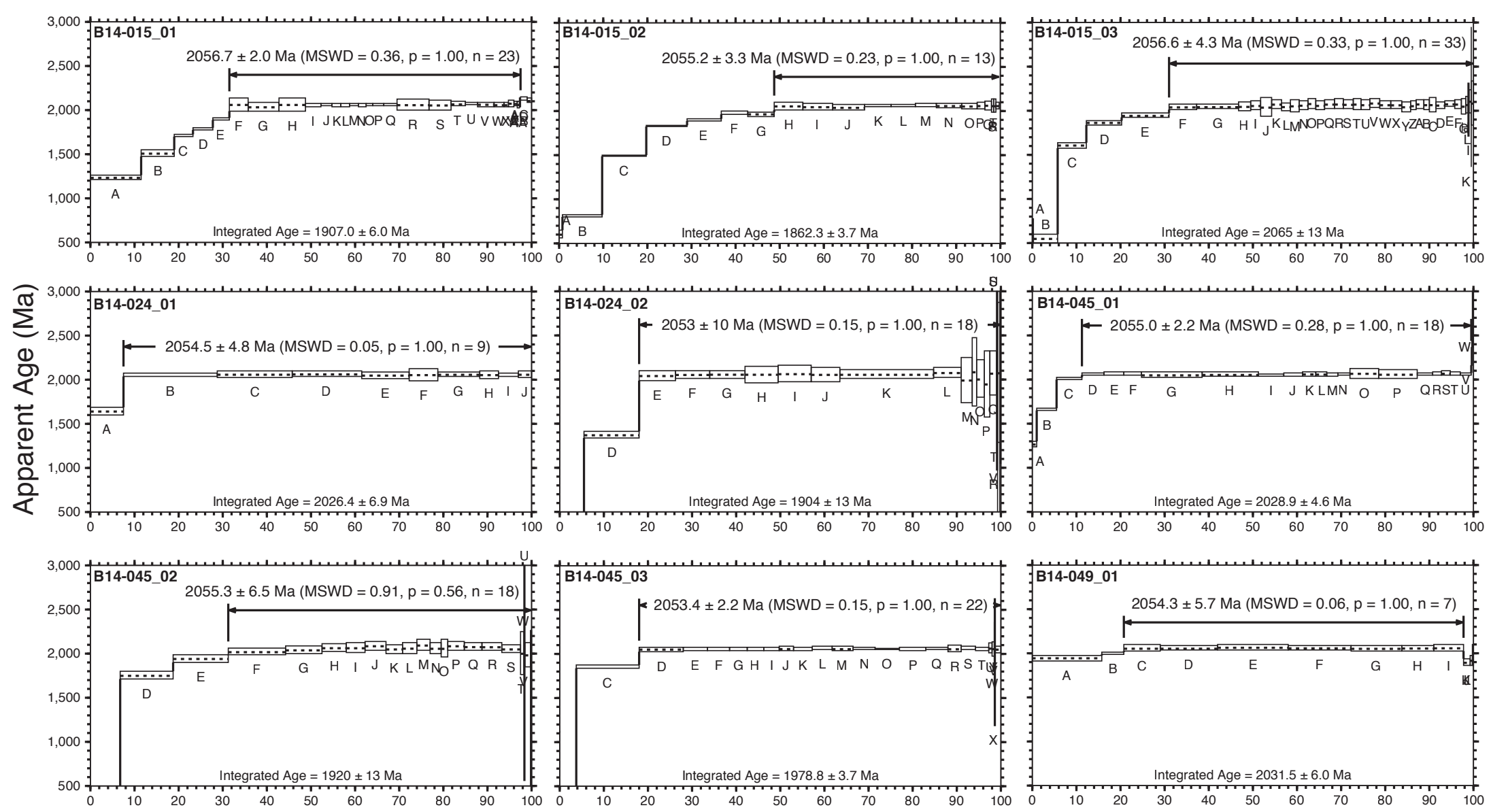

Cumulative $\%{ }^{39} \mathrm{Ar}$ Released

\section{Figure DR3: All Release Spectra. (Continued)}



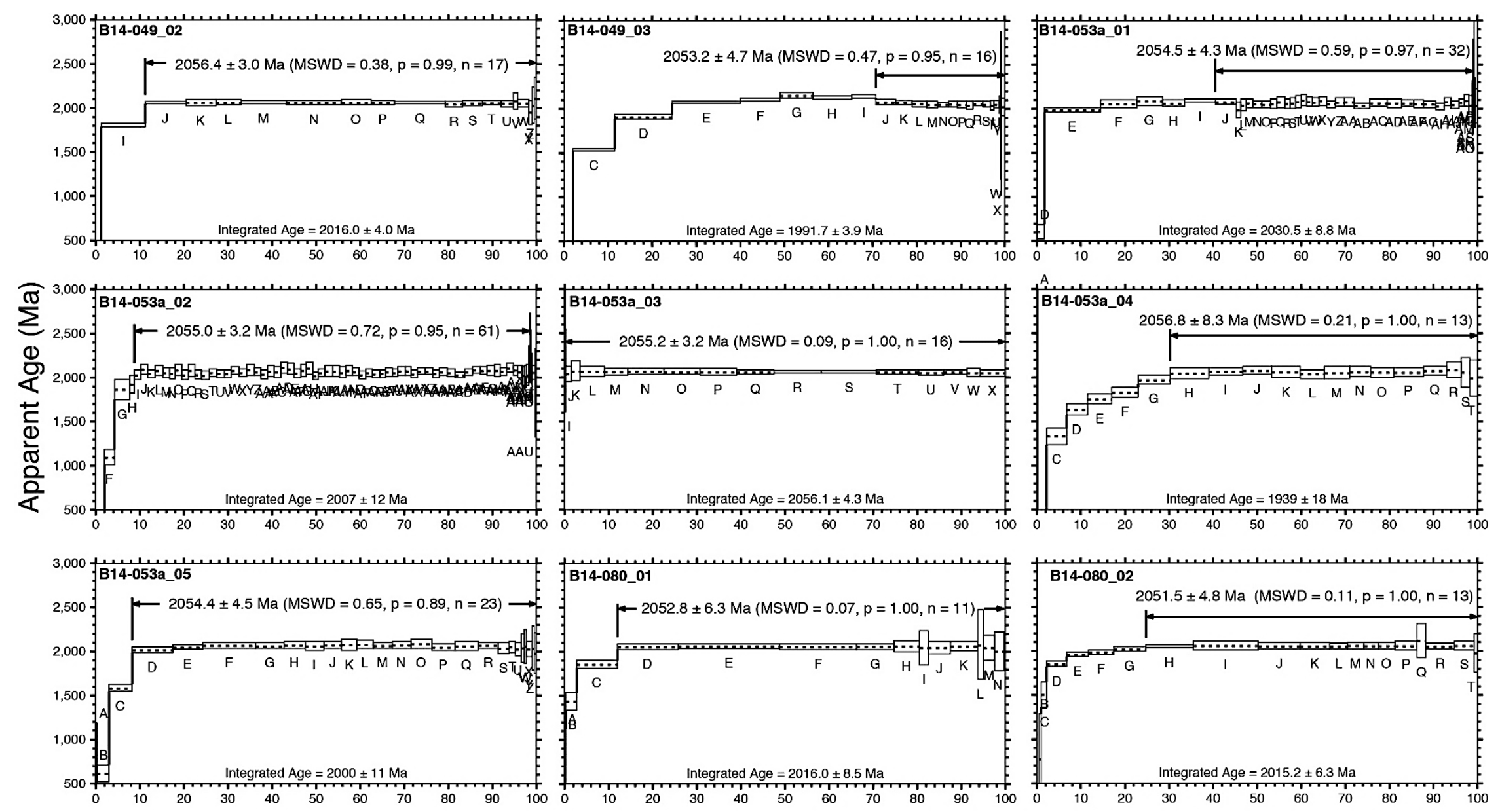

\section{Cumulative $\%{ }^{39} \mathrm{Ar}$ Released}

Figure DR3: All Release Spectra. (Continued) 

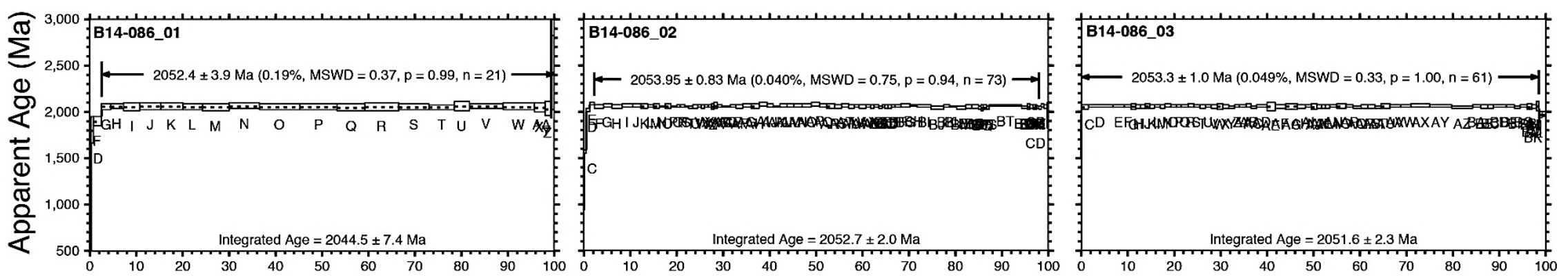

Cumulative $\%{ }^{39} \mathrm{Ar}$ Released

Figure DR3: All Release Spectra. (Continued) 


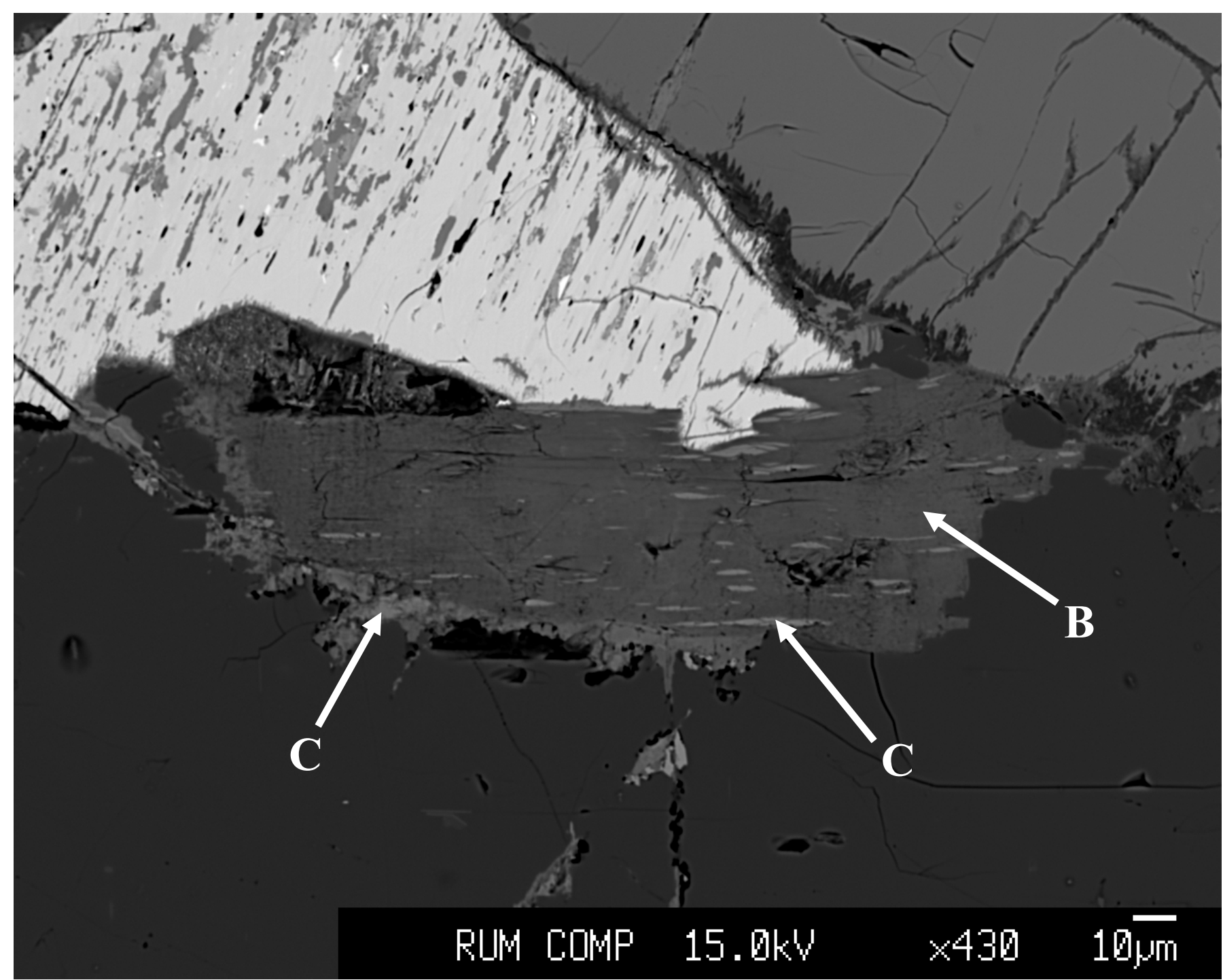

Figure DR4. Backscatter electron image of interlayered chlorite from B14-024. Arrows denote biotite (B) grain and areas of interlayered chlorite $(\mathrm{C})$. 


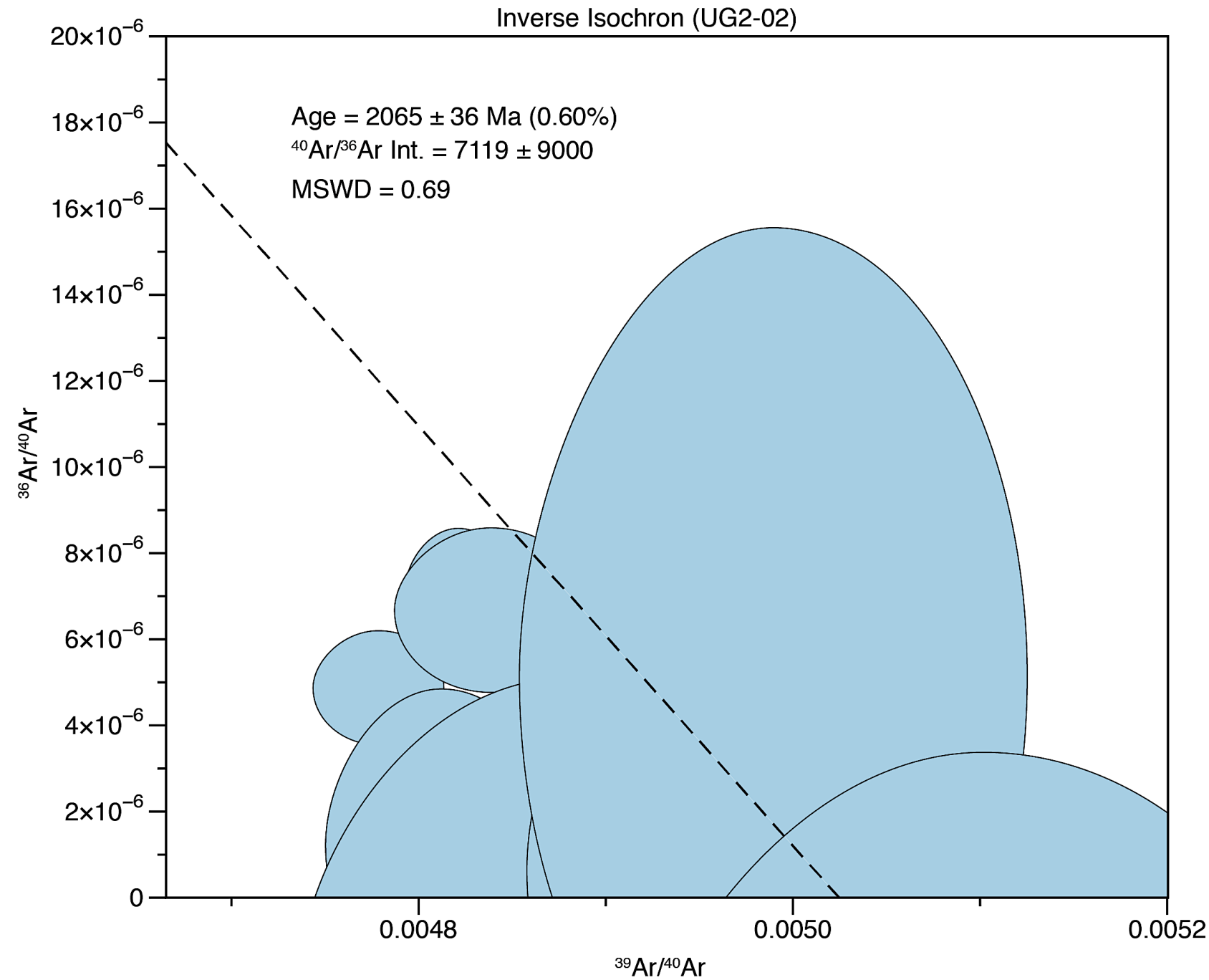

Figure DR5. Inverse Isochron from sample UG2-02 of Nomade et al. (2004). UG2-01 of Nomade et al. (2004) is an example of a discordant age spectra dominated by interlayered chlorite. The inverse isochron was constructed for steps $4.5 \mathrm{~W}$ (watts) through $10 \mathrm{~W}$ using Isoplot (Ludwig, 2003). The original reported plateau age for the sample was $2102 \mathrm{Ma}$, recalculated to $2110 \mathrm{Ma}$ to match the FC age of this study. 
Table DR1. Stepwise Heating Results: moles $\left(10^{-17}\right.$ for ${ }^{36,38,39} \mathrm{Ar}, 10^{-15}$ for ${ }^{40} \mathrm{Ar}$ and $\left.{ }^{40} \mathrm{Ar} *\right)$ of each isotope for each heating step. $\mathrm{J}$ is the measure of the neutron flus in the reactor. All uncertainties reported at $1 \sigma$. Blank cells represent negative values.

\begin{tabular}{|c|c|c|c|c|c|c|c|c|c|c|}
\hline Sample & Run ID & Power & $36 \mathrm{Ar}$ & $38 \mathrm{Ar}$ & 39Ar & $40 \mathrm{Ar}$ & 40Ar* & Age (Ma) & $\mathrm{R}_{\mathrm{i}}$ & $\begin{array}{c}\text { Used } \\
\text { in } \\
\text { Plateau } \\
\text { Age } \\
\end{array}$ \\
\hline B07-041_01 & $22486-01 \mathrm{~A}$ & 1.5 & $1.54 \pm 0.23$ & $0.89 \pm 0.10$ & $2.69 \pm 1.07$ & $6.62 \pm 1.11$ & $1.51 \pm 0.79$ & $1350 \pm 630$ & 70.67 & \\
\hline$J=1.98874 \mathrm{e}-2$ & 22486-01B & 3 & $3.66 \pm 0.24$ & $2.85 \pm 0.14$ & $12.47 \pm 1.13$ & $19.18 \pm 1.14$ & $7.00 \pm 0.82$ & $1350 \pm 140$ & 70.67 & \\
\hline \multirow[t]{21}{*}{ $\pm 3.18272 \mathrm{e}-5$} & 22486-01C & 5 & $6.60 \pm 0.25$ & $3.31 \pm 0.15$ & $26.29 \pm 1.04$ & $42.70 \pm 1.17$ & $20.74 \pm 0.84$ & $1702 \pm 64$ & 99.57 & \\
\hline & 22486-01D & 7 & $4.65 \pm 0.25$ & $4.65 \pm 0.18$ & $55.86 \pm 1.17$ & $74.51 \pm 1.18$ & $59.03 \pm 0.84$ & $2042 \pm 30$ & 133.39 & $\mathrm{x}$ \\
\hline & 22486-01E & 8 & $1.22 \pm 0.23$ & $3.52 \pm 0.15$ & $56.10 \pm 1.17$ & $64.91 \pm 1.11$ & $60.85 \pm 0.80$ & $2074 \pm 29$ & 136.91 & $\mathrm{x}$ \\
\hline & 22486-01F & 9 & $0.73 \pm 0.23$ & $4.31 \pm 0.19$ & $67.16 \pm 1.22$ & $76.02 \pm 1.11$ & $73.59 \pm 0.79$ & $2087 \pm 24$ & 138.36 & $\mathrm{x}$ \\
\hline & 22486-01G & 10 & $0.45 \pm 0.23$ & $4.79 \pm 0.17$ & $84.88 \pm 1.32$ & $93.78 \pm 1.10$ & $92.30 \pm 0.79$ & $2077 \pm 20$ & 137.24 & $\mathrm{x}$ \\
\hline & $22486-01 \mathrm{H}$ & 11 & $0.15 \pm 0.23$ & $5.37 \pm 0.19$ & $92.98 \pm 1.25$ & $100.01 \pm 1.11$ & $99.51 \pm 0.81$ & $2058 \pm 17$ & 135.14 & $\mathrm{x}$ \\
\hline & 22486-01I & 12 & $0.15 \pm 0.23$ & $5.25 \pm 0.19$ & $93.01 \pm 1.25$ & $99.92 \pm 1.10$ & $99.41 \pm 0.79$ & $2056 \pm 17$ & 134.92 & $\mathrm{x}$ \\
\hline & $22486-01 \mathrm{~J}$ & 13 & $0.23 \pm 0.23$ & $7.84 \pm 0.20$ & $98.12 \pm 1.28$ & $105.97 \pm 1.09$ & $105.20 \pm 0.78$ & $2060 \pm 16$ & 135.36 & $\mathrm{x}$ \\
\hline & 22486-01K & 14 & $0.11 \pm 0.23$ & $8.59 \pm 0.20$ & $107.40 \pm 1.32$ & $115.57 \pm 1.09$ & $115.20 \pm 0.78$ & $2060 \pm 15$ & 135.36 & $\mathrm{x}$ \\
\hline & 22486-01L & 15 & $0.22 \pm 0.23$ & $5.82 \pm 0.18$ & $99.26 \pm 1.26$ & $106.23 \pm 1.09$ & $105.50 \pm 0.78$ & $2049 \pm 15$ & 134.15 & $\mathrm{x}$ \\
\hline & $22486-01 \mathrm{M}$ & 16 & $0.19 \pm 0.22$ & $4.81 \pm 0.21$ & $79.38 \pm 1.27$ & $85.97 \pm 1.07$ & $85.33 \pm 0.76$ & $2063 \pm 21$ & 135.69 & $\mathrm{x}$ \\
\hline & $22486-01 \mathrm{~N}$ & 17 & $0.12 \pm 0.22$ & $2.81 \pm 0.17$ & $53.93 \pm 1.37$ & $58.03 \pm 1.07$ & $57.65 \pm 0.76$ & $2056 \pm 34$ & 134.92 & $\mathrm{x}$ \\
\hline & 22486-01O & 18 & $0.11 \pm 0.22$ & $3.78 \pm 0.17$ & $63.36 \pm 1.45$ & $68.64 \pm 1.06$ & $68.29 \pm 0.76$ & $2066 \pm 30$ & 136.02 & $\mathrm{x}$ \\
\hline & 22486-01P & 19 & $0.11 \pm 0.07$ & $3.60 \pm 0.15$ & $62.38 \pm 0.76$ & $66.81 \pm 0.35$ & $66.44 \pm 0.25$ & $2052 \pm 12$ & 134.48 & $\mathrm{x}$ \\
\hline & 22486-01Q & 20 & $0.06 \pm 0.07$ & $2.67 \pm 0.13$ & $46.51 \pm 0.66$ & $49.86 \pm 0.36$ & $49.67 \pm 0.27$ & $2055 \pm 16$ & 134.81 & $\mathrm{x}$ \\
\hline & 22486-01R & 22 & $0.19 \pm 0.08$ & $5.09 \pm 0.18$ & $77.86 \pm 0.86$ & $83.51 \pm 0.37$ & $82.86 \pm 0.27$ & $2051 \pm 10$ & 134.37 & $\mathrm{x}$ \\
\hline & $22486-01 \mathrm{~S}$ & 24 & $0.04 \pm 0.08$ & $8.70 \pm 0.22$ & $130.30 \pm 1.36$ & $139.45 \pm 0.42$ & $139.30 \pm 0.31$ & $2055.7 \pm 8.6$ & 134.89 & $\mathrm{x}$ \\
\hline & 22486-01T & 26 & $0.16 \pm 0.08$ & $4.97 \pm 0.17$ & $82.37 \pm 0.96$ & $88.65 \pm 0.39$ & $88.13 \pm 0.28$ & $2057 \pm 11$ & 135.03 & $\mathrm{x}$ \\
\hline & 22486-01U & 28 & $0.13 \pm 0.08$ & $4.26 \pm 0.16$ & $67.29 \pm 0.80$ & $72.17 \pm 0.37$ & $71.74 \pm 0.27$ & $2053 \pm 12$ & 134.59 & $\mathrm{x}$ \\
\hline & $22486-01 \mathrm{~V}$ & 30 & $0.36 \pm 0.08$ & $4.68 \pm 0.17$ & $88.75 \pm 1.00$ & $95.67 \pm 0.40$ & $94.45 \pm 0.30$ & $2051 \pm 10$ & 134.37 & $\mathrm{x}$ \\
\hline & 22486-01W & 32 & $0.24 \pm 0.07$ & $2.72 \pm 0.12$ & $45.01 \pm 0.64$ & $48.63 \pm 0.36$ & $47.82 \pm 0.26$ & $2049 \pm 16$ & 134.15 & $\mathrm{x}$ \\
\hline
\end{tabular}




\begin{tabular}{|c|c|c|c|c|c|c|c|c|c|}
\hline B07-041_02 & $22486-02 \mathrm{~A}$ & 1.5 & $1.37 \pm 0.08$ & $0.25 \pm 0.08$ & $5.32 \pm 0.45$ & $5.73 \pm 0.33$ & $1.72 \pm 0.24$ & $890 \pm 110$ & 40.48 \\
\hline$J=1.98874 \mathrm{e}-2$ & 22486-02B & 3 & $2.86 \pm 0.09$ & $0.87 \pm 0.10$ & $12.91 \pm 0.41$ & $17.13 \pm 0.37$ & $8.78 \pm 0.26$ & $1543 \pm 46$ & 85.82 \\
\hline \multirow[t]{20}{*}{ $\pm 3.18272 \mathrm{e}-5$} & $22486-02 \mathrm{C}$ & 5 & $5.29 \pm 0.12$ & $2.18 \pm 0.14$ & $58.36 \pm 0.57$ & $71.99 \pm 0.49$ & $56.56 \pm 0.35$ & $1938 \pm 13$ & 122.36 \\
\hline & 22486-02D & 7 & $0.82 \pm 0.09$ & $2.89 \pm 0.15$ & $152.70 \pm 0.95$ & $164.69 \pm 0.39$ & $162.30 \pm 0.29$ & $2049.0 \pm 6.6$ & 134.15 \\
\hline & $22486-02 \mathrm{E}$ & 8 & $0.09 \pm 0.13$ & $2.77 \pm 0.16$ & $139.90 \pm 1.34$ & $152.95 \pm 0.55$ & $152.70 \pm 0.40$ & $2081 \pm 11$ & 137.69 \\
\hline & $22486-02 \mathrm{~F}$ & 9 & $0.17 \pm 0.09$ & $3.01 \pm 0.17$ & $158.00 \pm 0.99$ & $168.78 \pm 0.40$ & $168.30 \pm 0.31$ & $2051.6 \pm 6.8$ & 134.44 \\
\hline & $22486-02 \mathrm{G}$ & 10 & $0.16 \pm 0.09$ & $2.72 \pm 0.16$ & $161.60 \pm 1.02$ & $173.16 \pm 0.40$ & $172.70 \pm 0.30$ & $2055.6 \pm 6.8$ & 134.88 \\
\hline & $22486-02 \mathrm{H}$ & 11 & $0.12 \pm 0.09$ & $2.75 \pm 0.15$ & $139.80 \pm 0.92$ & $150.16 \pm 0.40$ & $149.80 \pm 0.30$ & $2059.6 \pm 7.4$ & 135.32 \\
\hline & 22486-02I & 12 & $0.05 \pm 0.09$ & $2.39 \pm 0.17$ & $133.80 \pm 0.89$ & $143.16 \pm 0.40$ & $143.00 \pm 0.30$ & $2055.5 \pm 7.5$ & 134.87 \\
\hline & $22486-02 \mathrm{~J}$ & 13 & $0.38 \pm 0.14$ & $2.07 \pm 0.16$ & $126.60 \pm 0.89$ & $136.70 \pm 0.57$ & $135.60 \pm 0.41$ & $2058.1 \pm 8.5$ & 135.15 \\
\hline & $22486-02 \mathrm{~K}$ & 14 & $0.08 \pm 0.09$ & $1.94 \pm 0.17$ & $121.10 \pm 0.81$ & $129.32 \pm 0.45$ & $129.10 \pm 0.37$ & $2052.2 \pm 7.9$ & 134.50 \\
\hline & $22486-02 \mathrm{~L}$ & 15 & & $1.72 \pm 0.13$ & $102.10 \pm 0.79$ & $108.56 \pm 0.57$ & $109.00 \pm 0.42$ & $2053.8 \pm 9.8$ & 134.68 \\
\hline & $22486-02 \mathrm{M}$ & 16 & & $1.24 \pm 0.12$ & $63.07 \pm 0.62$ & $66.27 \pm 0.50$ & $66.75 \pm 0.38$ & $2044 \pm 13$ & 133.61 \\
\hline & $22486-02 \mathrm{~N}$ & 17 & & $0.54 \pm 0.10$ & $31.83 \pm 0.54$ & $33.05 \pm 0.46$ & $33.87 \pm 0.34$ & $2050 \pm 24$ & 134.26 \\
\hline & $22486-02 \mathrm{O}$ & 19 & $0.04 \pm 0.07$ & $1.20 \pm 0.12$ & $72.63 \pm 0.70$ & $77.37 \pm 0.32$ & $77.26 \pm 0.23$ & $2050 \pm 12$ & 134.26 \\
\hline & $22486-02 \mathrm{P}$ & 21 & & $1.33 \pm 0.14$ & $80.60 \pm 0.61$ & $85.39 \pm 0.49$ & $86.13 \pm 0.36$ & $2055.8 \pm 9.7$ & 134.90 \\
\hline & 22486-02Q & 23 & & $1.64 \pm 0.12$ & $90.69 \pm 0.73$ & $96.05 \pm 0.43$ & $96.68 \pm 0.31$ & $2052.8 \pm 9.7$ & 134.57 \\
\hline & $22486-02 R$ & 25 & & $2.00 \pm 0.15$ & $122.70 \pm 0.86$ & $129.83 \pm 0.35$ & $130.00 \pm 0.27$ & $2045.4 \pm 7.8$ & 133.76 \\
\hline & $22486-02 \mathrm{~S}$ & 27 & & $1.12 \pm 0.10$ & $69.94 \pm 0.67$ & $74.99 \pm 0.33$ & $75.03 \pm 0.24$ & $2061 \pm 12$ & 135.47 \\
\hline & $22486-02 \mathrm{~T}$ & 29 & $0.20 \pm 0.07$ & $0.56 \pm 0.10$ & $24.05 \pm 0.69$ & $26.18 \pm 0.30$ & $25.61 \pm 0.21$ & $2052 \pm 36$ & 134.48 \\
\hline & $22486-02 U$ & 31 & $0.10 \pm 0.07$ & $1.13 \pm 0.12$ & $56.29 \pm 0.59$ & $60.66 \pm 0.31$ & $60.38 \pm 0.23$ & $2060 \pm 13$ & 135.36 \\
\hline & $22486-02 \mathrm{~V}$ & 33 & $0.05 \pm 0.08$ & $0.20 \pm 0.10$ & $22.68 \pm 0.44$ & $24.14 \pm 0.32$ & $23.99 \pm 0.23$ & $2043 \pm 26$ & 133.50 \\
\hline B07-041_03 & $22486-03 \mathrm{~A}$ & 1.5 & $2.10 \pm 0.14$ & $0.63 \pm 0.10$ & $4.55 \pm 0.42$ & $8.83 \pm 0.71$ & $1.26 \pm 0.50$ & $790 \pm 260$ & 34.88 \\
\hline$J=1.98874 \mathrm{e}-2$ & 22486-03B & 3 & $2.43 \pm 0.10$ & $1.67 \pm 0.12$ & $53.92 \pm 0.91$ & $55.15 \pm 0.50$ & $46.39 \pm 0.36$ & $1799 \pm 20$ & 108.58 \\
\hline \multirow[t]{4}{*}{ $\pm 3.18272 \mathrm{e}-5$} & $22486-03 \mathrm{C}$ & 5 & $0.25 \pm 0.10$ & $3.02 \pm 0.15$ & $126.10 \pm 0.95$ & $136.61 \pm 0.51$ & $135.70 \pm 0.37$ & $2064.9 \pm 7.6$ & 135.90 \\
\hline & 22486-03D & 7 & $0.37 \pm 0.11$ & $5.16 \pm 0.21$ & $214.40 \pm 1.43$ & $230.54 \pm 0.57$ & $229.20 \pm 0.42$ & $2056.4 \pm 5.9$ & 134.97 \\
\hline & $22486-03 E$ & 8 & $0.41 \pm 0.10$ & $3.32 \pm 0.16$ & $129.90 \pm 1.01$ & $139.08 \pm 0.53$ & $137.60 \pm 0.38$ & $2045.3 \pm 8.1$ & 133.75 \\
\hline & $22486-03 \mathrm{~F}$ & 9 & $0.34 \pm 0.10$ & $2.27 \pm 0.14$ & $81.70 \pm 1.24$ & $88.31 \pm 0.54$ & $87.10 \pm 0.40$ & $2053 \pm 19$ & 134.59 \\
\hline
\end{tabular}




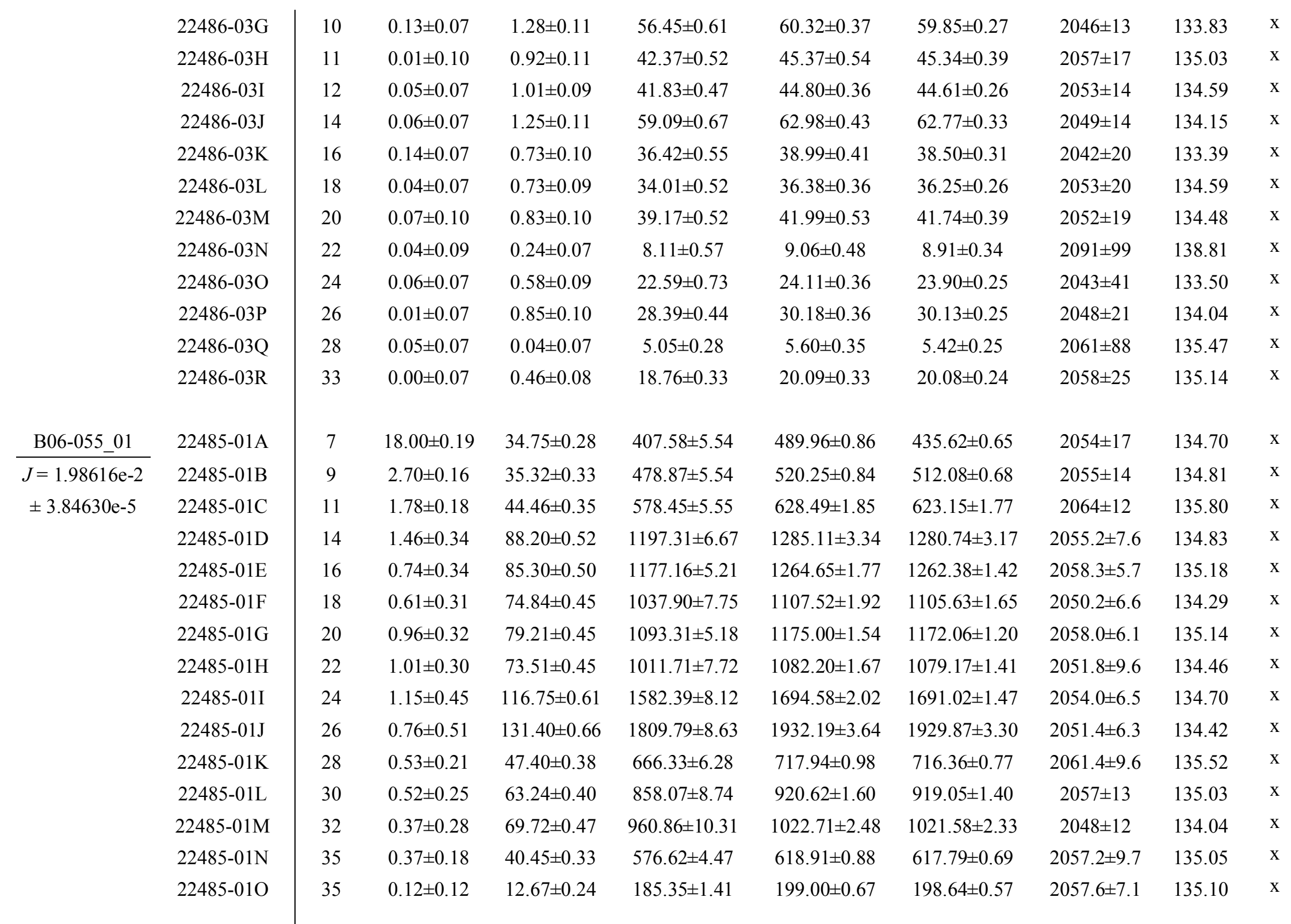




\begin{tabular}{|c|c|c|c|c|c|c|c|c|c|}
\hline B06-055-02 & $22485-02 \mathrm{~A}$ & 1.5 & $1.07 \pm 0.08$ & $0.62 \pm 0.09$ & $6.21 \pm 0.32$ & $7.78 \pm 0.32$ & $4.73 \pm 0.23$ & $1663 \pm 77$ & 96.09 \\
\hline$J=1.98616 \mathrm{e}-2$ & 22485-02B & 3 & $2.44 \pm 0.10$ & $3.22 \pm 0.14$ & $34.97 \pm 0.59$ & $44.34 \pm 0.40$ & $37.38 \pm 0.29$ & $2054 \pm 22$ & 134.70 \\
\hline \multirow[t]{27}{*}{ $\pm 3.84630 \mathrm{e}-5$} & $22485-02 \mathrm{C}$ & 5 & $0.22 \pm 0.13$ & $11.19 \pm 0.18$ & $152.80 \pm 1.12$ & $164.62 \pm 0.53$ & $164.00 \pm 0.38$ & $2059.4 \pm 8.2$ & 135.30 \\
\hline & 22485-02D & 6 & $0.06 \pm 0.09$ & $12.18 \pm 0.21$ & $169.90 \pm 1.20$ & $181.57 \pm 0.40$ & $181.40 \pm 0.30$ & $2052.5 \pm 7.6$ & 134.54 \\
\hline & $22485-02 \mathrm{E}$ & 7 & $0.10 \pm 0.11$ & $14.72 \pm 0.19$ & $158.70 \pm 0.99$ & $170.58 \pm 0.59$ & $170.30 \pm 0.50$ & $2059.7 \pm 7.1$ & 135.33 \\
\hline & $22485-02 \mathrm{~F}$ & 8 & $0.01 \pm 0.10$ & $15.99 \pm 0.21$ & $176.67 \pm 1.15$ & $188.98 \pm 0.54$ & $188.94 \pm 0.40$ & $2055.1 \pm 7.1$ & 134.82 \\
\hline & $22485-02 \mathrm{G}$ & 8.5 & $0.14 \pm 0.14$ & $12.31 \pm 0.22$ & $180.30 \pm 2.76$ & $192.20 \pm 0.71$ & $191.80 \pm 0.58$ & $2048 \pm 19$ & 134.04 \\
\hline & $22485-02 \mathrm{H}$ & 9 & $0.15 \pm 0.13$ & $9.70 \pm 0.19$ & $138.80 \pm 1.43$ & $148.43 \pm 0.53$ & $148.00 \pm 0.39$ & $2051 \pm 12$ & 134.37 \\
\hline & 22485-02I & 9.5 & $0.23 \pm 0.11$ & $9.61 \pm 0.23$ & $132.90 \pm 0.88$ & $143.05 \pm 0.46$ & $142.40 \pm 0.34$ & $2057.2 \pm 7.4$ & 135.05 \\
\hline & $22485-02 \mathrm{~J}$ & 10 & $0.03 \pm 0.08$ & $9.81 \pm 0.20$ & $136.20 \pm 0.92$ & $145.98 \pm 0.42$ & $145.90 \pm 0.35$ & $2056.9 \pm 7.7$ & 135.02 \\
\hline & $22485-02 \mathrm{~K}$ & 10.5 & $0.06 \pm 0.08$ & $8.58 \pm 0.20$ & $123.20 \pm 0.89$ & $131.99 \pm 0.32$ & $131.80 \pm 0.24$ & $2055.2 \pm 8.1$ & 134.83 \\
\hline & $22485-02 \mathrm{~L}$ & 11 & $0.15 \pm 0.08$ & $7.89 \pm 0.17$ & $115.80 \pm 0.79$ & $124.14 \pm 0.37$ & $123.70 \pm 0.28$ & $2053.5 \pm 7.7$ & 134.65 \\
\hline & $22485-02 \mathrm{M}$ & 11.5 & $0.18 \pm 0.09$ & $8.10 \pm 0.20$ & $113.10 \pm 0.93$ & $121.30 \pm 0.37$ & $120.80 \pm 0.27$ & $2054.1 \pm 9.5$ & 134.71 \\
\hline & $22485-02 \mathrm{~N}$ & 12 & $0.13 \pm 0.08$ & $6.61 \pm 0.21$ & $100.20 \pm 0.84$ & $107.86 \pm 0.38$ & $107.50 \pm 0.30$ & $2059 \pm 10$ & 135.25 \\
\hline & $22485-02 \mathrm{O}$ & 12.5 & $0.14 \pm 0.08$ & $7.73 \pm 0.17$ & $108.50 \pm 0.88$ & $116.39 \pm 0.35$ & $116.00 \pm 0.26$ & $2054.5 \pm 9.4$ & 134.76 \\
\hline & $22485-02 \mathrm{P}$ & 13 & $0.05 \pm 0.08$ & $9.20 \pm 0.21$ & $124.40 \pm 0.94$ & $133.04 \pm 0.41$ & $132.90 \pm 0.34$ & $2054.3 \pm 8.8$ & 134.74 \\
\hline & 22485-02Q & 13.5 & & $7.10 \pm 0.18$ & $99.66 \pm 0.79$ & $106.52 \pm 0.34$ & $106.70 \pm 0.26$ & $2056.5 \pm 9.3$ & 134.98 \\
\hline & $22485-02 R$ & 14 & $0.00 \pm 0.13$ & $10.95 \pm 0.23$ & $151.10 \pm 1.00$ & $161.40 \pm 0.66$ & $161.40 \pm 0.55$ & $2053.8 \pm 8.1$ & 134.68 \\
\hline & $22485-02 S$ & 14.5 & $0.05 \pm 0.08$ & $7.87 \pm 0.19$ & $113.00 \pm 1.32$ & $120.64 \pm 0.38$ & $120.50 \pm 0.30$ & $2051 \pm 14$ & 134.37 \\
\hline & $22485-02 \mathrm{~T}$ & 15 & $0.19 \pm 0.09$ & $9.63 \pm 0.22$ & $141.80 \pm 0.91$ & $152.53 \pm 0.37$ & $152.00 \pm 0.27$ & $2058.7 \pm 7.0$ & 135.22 \\
\hline & $22485-02 U$ & 15.5 & $0.17 \pm 0.08$ & $7.70 \pm 0.20$ & $107.60 \pm 0.80$ & $116.27 \pm 0.40$ & $115.80 \pm 0.33$ & $2062.0 \pm 8.8$ & 135.58 \\
\hline & $22485-02 \mathrm{~V}$ & 16 & $0.08 \pm 0.08$ & $9.88 \pm 0.20$ & $142.70 \pm 1.59$ & $153.02 \pm 0.35$ & $152.80 \pm 0.26$ & $2056 \pm 13$ & 134.92 \\
\hline & $22485-02 \mathrm{~W}$ & 16.5 & $0.04 \pm 0.08$ & $3.76 \pm 0.16$ & $54.37 \pm 0.68$ & $58.78 \pm 0.43$ & $58.66 \pm 0.36$ & $2066 \pm 17$ & 136.02 \\
\hline & $22485-02 X$ & 17 & $0.03 \pm 0.08$ & $6.53 \pm 0.19$ & $100.30 \pm 1.24$ & $107.69 \pm 0.38$ & $107.60 \pm 0.31$ & $2060 \pm 15$ & 135.36 \\
\hline & $22485-02 Y$ & 17.5 & $0.10 \pm 0.07$ & $4.82 \pm 0.16$ & $70.60 \pm 0.70$ & $75.49 \pm 0.37$ & $75.20 \pm 0.31$ & $2050 \pm 12$ & 134.26 \\
\hline & $22485-02 Z$ & 18 & $0.01 \pm 0.08$ & $4.79 \pm 0.15$ & $72.71 \pm 0.68$ & $77.48 \pm 0.36$ & $77.46 \pm 0.29$ & $2050 \pm 12$ & 134.26 \\
\hline & $22485-02 \mathrm{AA}$ & 18.5 & $0.20 \pm 0.11$ & $6.98 \pm 0.17$ & $99.87 \pm 0.81$ & $106.76 \pm 0.45$ & $106.20 \pm 0.33$ & $2048.0 \pm 9.7$ & 134.04 \\
\hline & $22485-02 \mathrm{AB}$ & 19 & $0.12 \pm 0.13$ & $4.67 \pm 0.17$ & $69.14 \pm 0.97$ & $74.32 \pm 0.57$ & $73.99 \pm 0.42$ & $2056 \pm 18$ & 134.92 \\
\hline & $22485-02 \mathrm{AC}$ & 19.5 & $0.13 \pm 0.10$ & $1.35 \pm 0.10$ & $22.25 \pm 0.74$ & $24.07 \pm 0.41$ & $23.70 \pm 0.30$ & $2050 \pm 43$ & 134.26 \\
\hline
\end{tabular}




\begin{tabular}{|c|c|c|c|c|c|c|c|c|c|c|}
\hline & $22485-02 \mathrm{AD}$ & 18.5 & $0.16 \pm 0.09$ & $1.06 \pm 0.10$ & $14.21 \pm 0.32$ & $15.68 \pm 0.39$ & $15.21 \pm 0.28$ & $2056 \pm 36$ & 134.92 & $\mathrm{x}$ \\
\hline & 22485-02AE & 20 & $0.18 \pm 0.10$ & $3.90 \pm 0.15$ & $58.14 \pm 0.56$ & $63.02 \pm 0.39$ & $62.50 \pm 0.28$ & $2061 \pm 12$ & 135.47 & $\mathrm{x}$ \\
\hline & $22485-02 \mathrm{AF}$ & 20.5 & $0.10 \pm 0.10$ & $0.86 \pm 0.09$ & $13.93 \pm 1.13$ & $14.68 \pm 0.41$ & $14.40 \pm 0.29$ & $2010 \pm 100$ & 129.93 & $\mathrm{x}$ \\
\hline & 22485-02AG & 22 & $0.02 \pm 0.08$ & $3.38 \pm 0.10$ & $50.83 \pm 0.55$ & $54.45 \pm 0.33$ & $54.41 \pm 0.25$ & $2056 \pm 14$ & 134.92 & $\mathrm{X}$ \\
\hline & 22485-02AH & 24 & $0.01 \pm 0.07$ & $1.69 \pm 0.10$ & $22.37 \pm 0.46$ & $23.92 \pm 0.31$ & $23.89 \pm 0.22$ & $2054 \pm 27$ & 134.70 & $\mathrm{x}$ \\
\hline & 22485-02AI & 26 & $0.06 \pm 0.10$ & $0.51 \pm 0.08$ & $8.68 \pm 0.58$ & $9.27 \pm 0.42$ & $9.09 \pm 0.30$ & $2030 \pm 91$ & 132.08 & $x$ \\
\hline & $22485-02 \mathrm{AJ}$ & 30 & $0.02 \pm 0.07$ & $2.51 \pm 0.14$ & $38.58 \pm 0.55$ & $41.52 \pm 0.29$ & $41.45 \pm 0.22$ & $2061 \pm 18$ & 135.47 & $\mathrm{x}$ \\
\hline & 22485-02AK & 33 & $0.02 \pm 0.07$ & $0.55 \pm 0.08$ & $7.41 \pm 0.62$ & $7.52 \pm 0.29$ & $7.45 \pm 0.21$ & $1980 \pm 110$ & 126.74 & $\mathrm{x}$ \\
\hline B06-055_03 & $22485-04 \mathrm{~A}$ & 1.5 & $1.80 \pm 0.09$ & $0.58 \pm 0.10$ & $5.43 \pm 0.42$ & $9.50 \pm 0.37$ & $4.35 \pm 0.26$ & $1720 \pm 110$ & 101.21 & \\
\hline$J=1.98616 \mathrm{e}-2$ & 22485-04B & 3 & $4.43 \pm 0.17$ & $1.74 \pm 0.11$ & $14.72 \pm 0.66$ & $28.50 \pm 0.69$ & $15.82 \pm 0.50$ & $2061 \pm 68$ & 135.47 & $\mathrm{x}$ \\
\hline $\pm 3.84630 \mathrm{e}-5$ & $22485-04 \mathrm{C}$ & 5 & $1.43 \pm 0.09$ & $3.62 \pm 0.17$ & $42.78 \pm 1.59$ & $49.75 \pm 0.37$ & $45.67 \pm 0.28$ & $2053 \pm 46$ & 134.59 & $\mathrm{x}$ \\
\hline & 22485-04D & 7 & $0.32 \pm 0.11$ & $6.44 \pm 0.18$ & $90.76 \pm 0.73$ & $97.58 \pm 0.46$ & $96.66 \pm 0.33$ & $2045.0 \pm 9.8$ & 133.72 & $\mathrm{x}$ \\
\hline & $22485-04 \mathrm{E}$ & 9 & $0.32 \pm 0.09$ & $11.72 \pm 0.24$ & $159.60 \pm 1.12$ & $171.02 \pm 0.43$ & $170.10 \pm 0.35$ & $2051.3 \pm 7.8$ & 134.41 & $\mathrm{x}$ \\
\hline & $22485-04 \mathrm{~F}$ & 10 & $0.22 \pm 0.08$ & $8.55 \pm 0.21$ & $117.60 \pm 1.15$ & $126.33 \pm 0.36$ & $125.70 \pm 0.29$ & $2054 \pm 12$ & 134.70 & $\mathrm{x}$ \\
\hline & $22485-04 \mathrm{G}$ & 11 & $0.16 \pm 0.08$ & $7.42 \pm 0.20$ & $103.00 \pm 0.83$ & $110.57 \pm 0.34$ & $110.10 \pm 0.26$ & $2054.3 \pm 9.3$ & 134.74 & $\mathrm{x}$ \\
\hline & $22485-04 \mathrm{H}$ & 12 & $0.03 \pm 0.08$ & $7.72 \pm 0.18$ & $99.37 \pm 1.22$ & $106.57 \pm 0.36$ & $106.50 \pm 0.29$ & $2058 \pm 15$ & 135.14 & $\mathrm{x}$ \\
\hline & 22485-04I & 13 & $0.19 \pm 0.08$ & $7.46 \pm 0.18$ & $103.30 \pm 0.79$ & $110.64 \pm 0.35$ & $110.10 \pm 0.26$ & $2050.4 \pm 8.7$ & 134.31 & $\mathrm{x}$ \\
\hline & $22485-04 \mathrm{~J}$ & 14 & $0.08 \pm 0.08$ & $8.60 \pm 0.23$ & $115.90 \pm 1.42$ & $124.34 \pm 0.38$ & $124.10 \pm 0.30$ & $2057 \pm 15$ & 135.03 & $\mathrm{x}$ \\
\hline & $22485-04 \mathrm{~K}$ & 15 & $0.06 \pm 0.11$ & $11.31 \pm 0.27$ & $148.60 \pm 0.97$ & $158.78 \pm 0.50$ & $158.60 \pm 0.38$ & $2052.5 \pm 7.2$ & 134.54 & $\mathrm{x}$ \\
\hline & $22485-04 \mathrm{~L}$ & 16 & $0.13 \pm 0.09$ & $9.67 \pm 0.21$ & $125.60 \pm 0.87$ & $135.46 \pm 0.39$ & $135.10 \pm 0.30$ & $2062.0 \pm 7.7$ & 135.58 & $\mathrm{x}$ \\
\hline & $22485-04 \mathrm{M}$ & 17 & $0.07 \pm 0.09$ & $8.41 \pm 0.18$ & $109.30 \pm 0.86$ & $117.31 \pm 0.41$ & $117.10 \pm 0.33$ & $2057.4 \pm 9.2$ & 135.08 & $\mathrm{x}$ \\
\hline & $22485-04 \mathrm{~N}$ & 18 & $0.15 \pm 0.12$ & $12.88 \pm 0.24$ & $171.80 \pm 1.08$ & $183.64 \pm 0.50$ & $183.20 \pm 0.37$ & $2051.3 \pm 6.6$ & 134.41 & $\mathrm{x}$ \\
\hline & $22485-04 \mathrm{O}$ & 19 & $0.52 \pm 0.15$ & $33.43 \pm 0.28$ & $347.10 \pm 2.41$ & $373.99 \pm 0.82$ & $372.50 \pm 0.71$ & $2059.3 \pm 7.4$ & 135.29 & $\mathrm{x}$ \\
\hline & $22485-04 \mathrm{P}$ & 20 & $0.15 \pm 0.08$ & $12.54 \pm 0.25$ & $156.80 \pm 1.53$ & $168.32 \pm 0.35$ & $167.90 \pm 0.26$ & $2057 \pm 11$ & 135.03 & $\mathrm{x}$ \\
\hline & 22485-04Q & 21 & $0.11 \pm 0.08$ & $9.35 \pm 0.26$ & $131.70 \pm 0.95$ & $141.92 \pm 0.38$ & $141.60 \pm 0.30$ & $2061.2 \pm 7.8$ & 135.49 & $\mathrm{x}$ \\
\hline & $22485-04 R$ & 22 & & $3.92 \pm 0.15$ & $56.73 \pm 0.67$ & $61.30 \pm 0.30$ & $61.43 \pm 0.22$ & $2071 \pm 14$ & 136.58 & $\mathrm{x}$ \\
\hline & $22485-04 \mathrm{~S}$ & 23 & $0.03 \pm 0.07$ & $5.88 \pm 0.16$ & $76.87 \pm 0.69$ & $82.23 \pm 0.30$ & $82.16 \pm 0.23$ & $2054 \pm 10$ & 134.70 & $\mathrm{x}$ \\
\hline & $22485-04 \mathrm{~T}$ & 24 & $0.13 \pm 0.08$ & $6.77 \pm 0.17$ & $92.48 \pm 0.66$ & $99.62 \pm 0.32$ & $99.24 \pm 0.24$ & $2059.2 \pm 9.3$ & 135.27 & $\mathrm{x}$ \\
\hline
\end{tabular}




\begin{tabular}{|c|c|c|c|c|c|c|c|c|c|}
\hline & $22485-04 \mathrm{U}$ & 25 & $0.11 \pm 0.07$ & $5.03 \pm 0.16$ & $67.76 \pm 0.47$ & $72.98 \pm 0.34$ & $72.66 \pm 0.27$ & $2058.5 \pm 9.8$ & 135.20 \\
\hline & $22485-04 \mathrm{~V}$ & 27 & $0.10 \pm 0.07$ & $2.67 \pm 0.13$ & $38.45 \pm 0.79$ & $41.26 \pm 0.30$ & $40.97 \pm 0.21$ & $2050 \pm 26$ & 134.26 \\
\hline & 22485-04W & 29 & $0.09 \pm 0.10$ & $1.29 \pm 0.10$ & $15.14 \pm 0.60$ & $16.57 \pm 0.42$ & $16.32 \pm 0.30$ & $2064 \pm 54$ & 135.80 \\
\hline & $22485-04 X$ & 31 & $0.03 \pm 0.07$ & $3.66 \pm 0.15$ & $47.82 \pm 0.53$ & $51.08 \pm 0.32$ & $50.98 \pm 0.24$ & $2051 \pm 15$ & 134.37 \\
\hline B06-042_01 & 22484-01A & 7 & $13.86 \pm 0.28$ & $80.07 \pm 0.58$ & $608.09 \pm 13.98$ & $682.68 \pm 1.18$ & $649.43 \pm 0.97$ & $2052 \pm 28$ & 134.48 \\
\hline$J=1.98464 \mathrm{e}-2$ & 22484-01B & 10 & $0.45 \pm 0.29$ & $119.09 \pm 0.60$ & $978.95 \pm 4.82$ & $1051.07 \pm 1.06$ & $1050.02 \pm 0.83$ & $2057.8 \pm 6.2$ & 135.12 \\
\hline \multirow[t]{13}{*}{ $\pm 3.84784 \mathrm{e}-5$} & 22484-01C & 12 & $0.12 \pm 0.24$ & $96.11 \pm 0.45$ & $801.71 \pm 5.17$ & $858.24 \pm 0.84$ & $857.99 \pm 0.68$ & $2055.0 \pm 8.0$ & 134.81 \\
\hline & 22484-01D & 14 & $0.14 \pm 0.25$ & $103.31 \pm 0.53$ & $832.19 \pm 8.44$ & $891.41 \pm 0.97$ & $891.06 \pm 0.70$ & $2056 \pm 13$ & 134.92 \\
\hline & 22484-01E & 16 & $0.14 \pm 0.29$ & $125.85 \pm 0.66$ & $996.38 \pm 5.53$ & $1069.39 \pm 1.15$ & $1069.07 \pm 0.93$ & $2058.2 \pm 7.0$ & 135.16 \\
\hline & $22484-01 \mathrm{~F}$ & 18 & $0.10 \pm 0.28$ & $115.64 \pm 0.58$ & $928.92 \pm 5.17$ & $995.24 \pm 0.89$ & $995.04 \pm 0.70$ & $2056.1 \pm 7.0$ & 134.93 \\
\hline & $22484-01 G$ & 20 & $0.50 \pm 0.35$ & $151.16 \pm 0.68$ & $1210.54 \pm 8.43$ & $1299.81 \pm 1.40$ & $1298.64 \pm 1.15$ & $2058.0 \pm 8.7$ & 135.14 \\
\hline & 22484-01H & 22 & $0.36 \pm 0.34$ & $148.56 \pm 0.66$ & $1179.18 \pm 5.17$ & $1264.98 \pm 1.21$ & $1264.10 \pm 0.88$ & $2057.2 \pm 5.6$ & 135.05 \\
\hline & 22484-01I & 24 & $0.46 \pm 0.44$ & $194.56 \pm 0.68$ & $1548.21 \pm 5.57$ & $1655.62 \pm 1.57$ & $1654.46 \pm 1.11$ & $2053.3 \pm 4.6$ & 134.63 \\
\hline & $22484-01 \mathrm{~J}$ & 26 & $0.55 \pm 0.36$ & $153.94 \pm 0.71$ & $1257.00 \pm 5.54$ & $1343.22 \pm 2.35$ & $1341.88 \pm 2.17$ & $2052.0 \pm 5.9$ & 134.48 \\
\hline & $22484-01 K$ & 28 & $0.09 \pm 0.19$ & $55.55 \pm 0.43$ & $453.24 \pm 7.67$ & $480.74 \pm 0.68$ & $480.54 \pm 0.53$ & $2044 \pm 21$ & 133.61 \\
\hline & 22484-01L & 30 & $0.48 \pm 0.26$ & $109.73 \pm 0.63$ & $890.60 \pm 8.42$ & $948.89 \pm 2.42$ & $947.75 \pm 2.34$ & $2048 \pm 12$ & 134.04 \\
\hline & $22484-01 M$ & 32 & $0.50 \pm 0.18$ & $71.41 \pm 0.50$ & $569.77 \pm 8.10$ & $610.30 \pm 1.49$ & $609.08 \pm 1.42$ & $2054 \pm 18$ & 134.70 \\
\hline & $22484-01 \mathrm{~N}$ & 35 & $0.22 \pm 0.13$ & $23.13 \pm 0.28$ & $193.10 \pm 0.89$ & $207.83 \pm 0.67$ & $207.30 \pm 0.59$ & $2058.7 \pm 6.7$ & 135.22 \\
\hline & $22484-010$ & 35 & $0.01 \pm 0.09$ & $2.13 \pm 0.13$ & $16.60 \pm 0.37$ & $17.50 \pm 0.31$ & $17.47 \pm 0.22$ & $2035 \pm 32$ & 132.63 \\
\hline B06-042_02 & $22484-02$ & 1.5 & $1.16 \pm 0.08$ & $1.96 \pm 0.11$ & $11.40 \pm 0.62$ & $11.04 \pm 0.32$ & $7.90 \pm 0.23$ & $1561 \pm 64$ & 87.32 \\
\hline$J=1.98464 \mathrm{e}-2$ & $22484-03$ & 3 & $4.53 \pm 0.11$ & $4.46 \pm 0.16$ & $29.53 \pm 0.64$ & $38.59 \pm 0.44$ & $26.35 \pm 0.31$ & $1839 \pm 28$ & 112.44 \\
\hline \multirow[t]{6}{*}{ $\pm 3.84784 \mathrm{e}-5$} & $22484-04$ & 4 & $0.42 \pm 0.08$ & $3.94 \pm 0.15$ & $27.63 \pm 0.72$ & $30.49 \pm 0.31$ & $29.36 \pm 0.23$ & $2046 \pm 33$ & 133.83 \\
\hline & $22484-05 \mathrm{~A}$ & 6 & $0.37 \pm 0.08$ & $12.36 \pm 0.22$ & $95.48 \pm 1.23$ & $103.59 \pm 0.40$ & $102.60 \pm 0.33$ & $2060 \pm 15$ & 135.36 \\
\hline & 22484-05B & 7.5 & $0.15 \pm 0.08$ & $14.18 \pm 0.26$ & $111.50 \pm 2.06$ & $120.00 \pm 0.33$ & $119.60 \pm 0.25$ & $2058 \pm 22$ & 135.14 \\
\hline & $22484-05 \mathrm{C}$ & 9 & $0.06 \pm 0.08$ & $17.32 \pm 0.25$ & $134.60 \pm 1.67$ & $144.36 \pm 0.36$ & $144.20 \pm 0.28$ & $2056 \pm 14$ & 134.92 \\
\hline & 22484-05D & 10 & $0.17 \pm 0.08$ & $15.38 \pm 0.27$ & $119.10 \pm 1.34$ & $129.25 \pm 0.85$ & $128.80 \pm 0.82$ & $2068 \pm 14$ & 136.25 \\
\hline & $22484-05 \mathrm{E}$ & 11 & $0.06 \pm 0.08$ & $14.80 \pm 0.26$ & $111.20 \pm 0.94$ & $119.46 \pm 0.36$ & $119.30 \pm 0.29$ & $2057.3 \pm 8.6$ & 135.06 \\
\hline
\end{tabular}




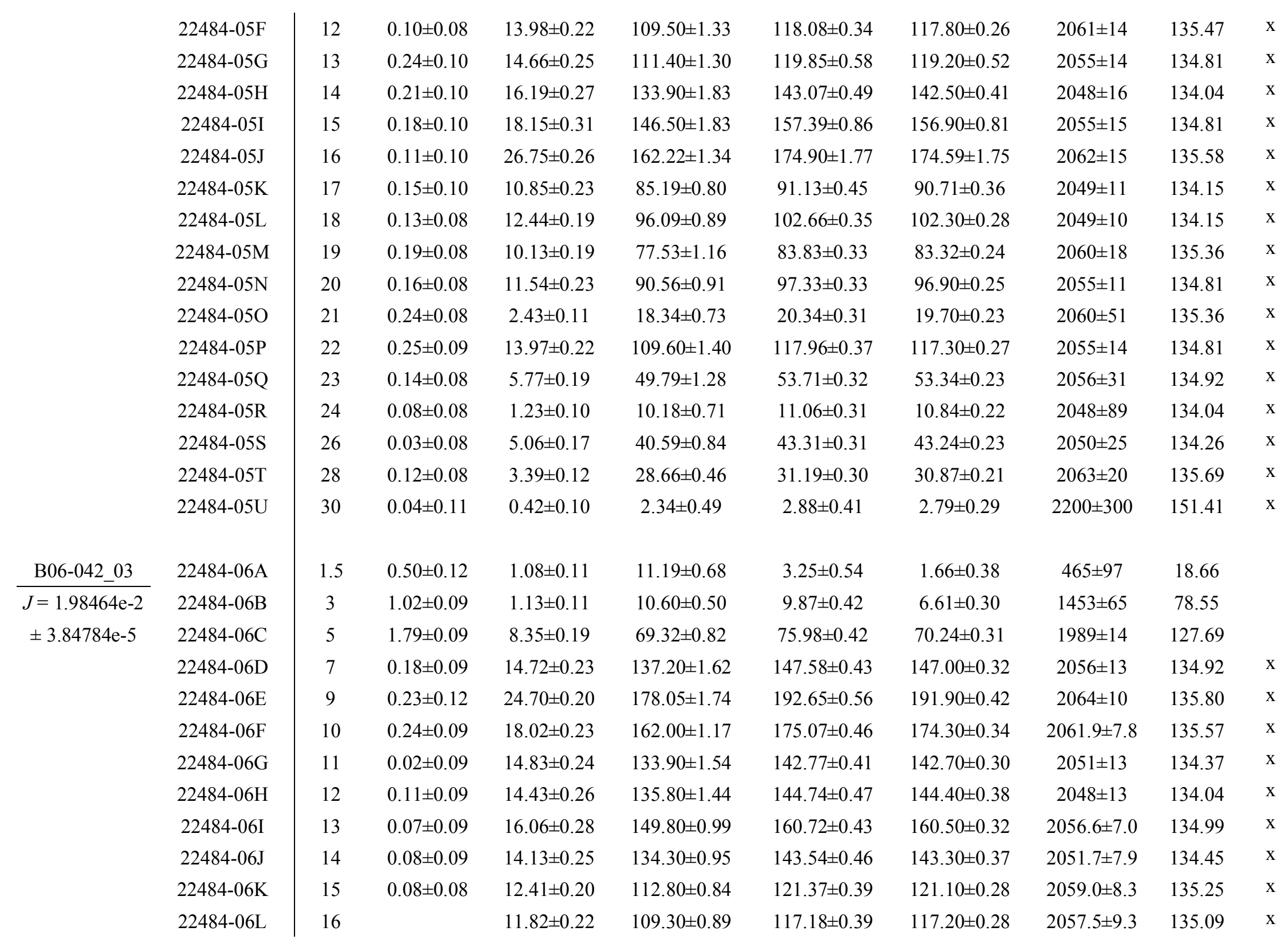




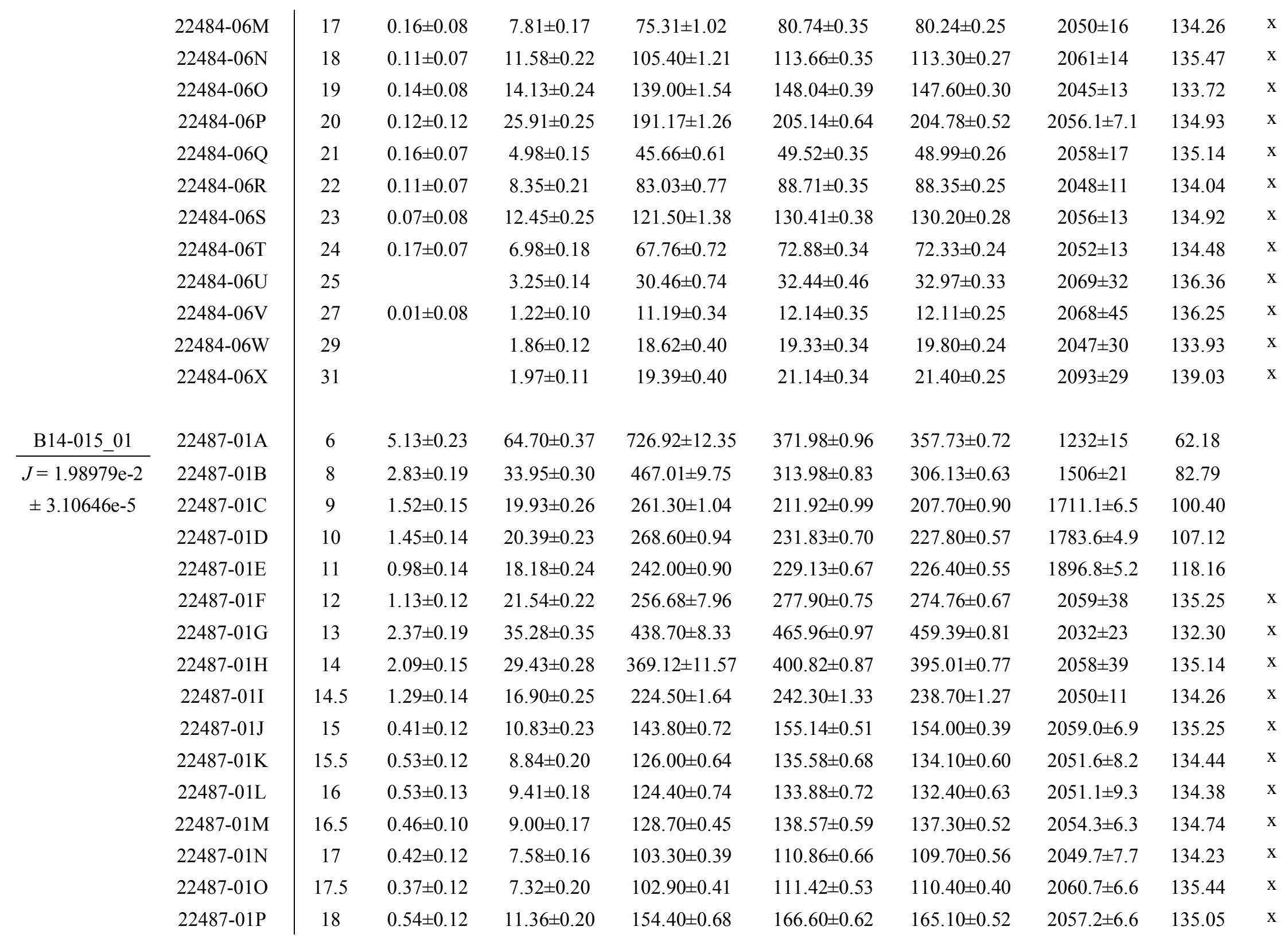




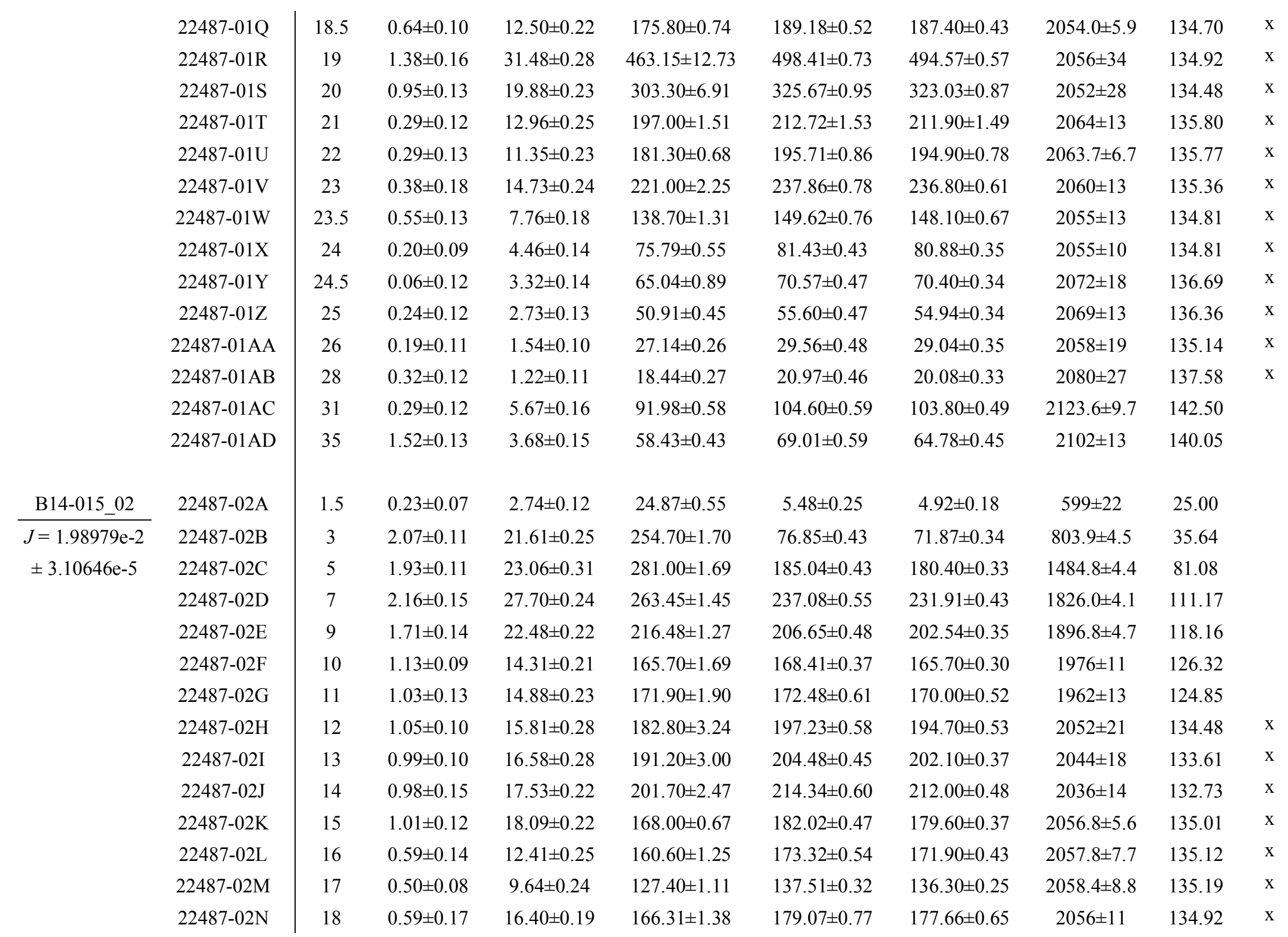




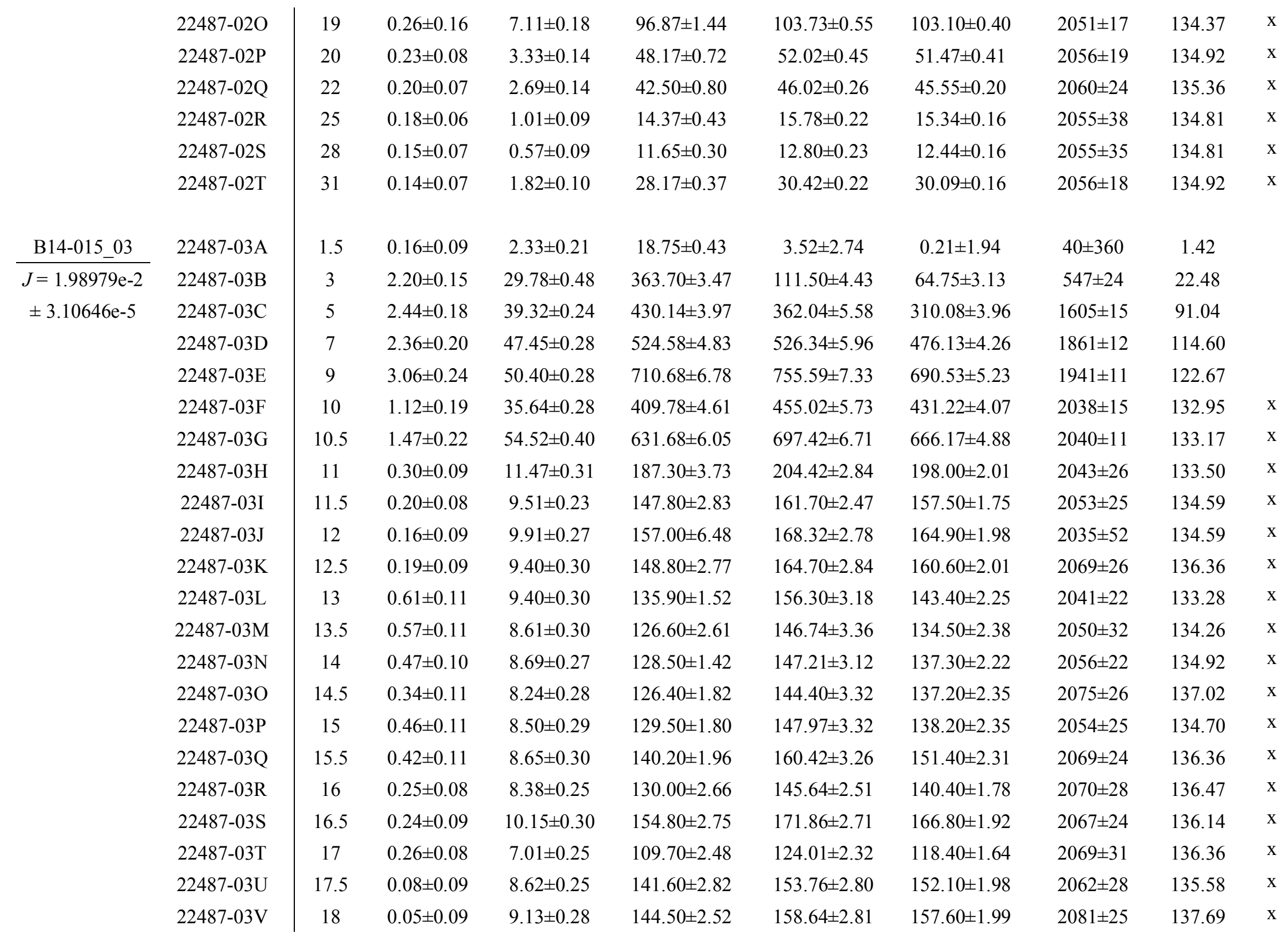




\begin{tabular}{|c|c|c|c|c|c|c|c|c|c|}
\hline & $22487-03 \mathrm{~W}$ & 18.5 & $0.07 \pm 0.10$ & $10.09 \pm 0.30$ & $169.00 \pm 2.89$ & $182.83 \pm 2.90$ & $181.40 \pm 2.05$ & $2062 \pm 23$ & 135.58 \\
\hline & $22487-03 X$ & 19 & $0.42 \pm 0.09$ & $10.05 \pm 0.30$ & $156.90 \pm 2.08$ & $177.29 \pm 2.82$ & $168.30 \pm 2.00$ & $2061 \pm 20$ & 135.47 \\
\hline & $22487-03 Y$ & 19.5 & $0.36 \pm 0.09$ & $8.30 \pm 0.24$ & $129.10 \pm 2.72$ & $143.74 \pm 2.71$ & $136.00 \pm 1.92$ & $2039 \pm 30$ & 133.06 \\
\hline & $22487-03 Z$ & 20 & $0.25 \pm 0.08$ & $6.02 \pm 0.25$ & $93.69 \pm 1.14$ & $105.00 \pm 2.57$ & $99.71 \pm 1.83$ & $2051 \pm 25$ & 134.37 \\
\hline & $22487-04 \mathrm{~A}$ & 20.5 & $0.08 \pm 0.08$ & $6.55 \pm 0.23$ & $104.60 \pm 1.56$ & $114.84 \pm 2.43$ & $113.10 \pm 1.72$ & $2071 \pm 24$ & 136.58 \\
\hline & 22487-04B & 21 & $0.16 \pm 0.08$ & $6.07 \pm 0.22$ & $93.23 \pm 1.48$ & $103.57 \pm 2.30$ & $100.10 \pm 1.64$ & $2063 \pm 26$ & 135.69 \\
\hline & $22487-04 \mathrm{C}$ & 21.5 & $0.11 \pm 0.08$ & $5.46 \pm 0.22$ & $84.80 \pm 2.20$ & $93.57 \pm 2.40$ & $91.12 \pm 1.70$ & $2063 \pm 38$ & 135.69 \\
\hline & 22487-04D & 22 & $0.30 \pm 0.08$ & $8.93 \pm 0.23$ & $134.80 \pm 1.73$ & $150.69 \pm 2.46$ & $144.30 \pm 1.74$ & $2058 \pm 19$ & 135.14 \\
\hline & $22487-04 \mathrm{E}$ & 23 & $0.30 \pm 0.09$ & $9.26 \pm 0.26$ & $149.90 \pm 1.60$ & $168.61 \pm 2.58$ & $162.30 \pm 1.83$ & $2072 \pm 16$ & 136.69 \\
\hline & $22487-04 \mathrm{~F}$ & 24 & $0.18 \pm 0.08$ & $5.88 \pm 0.24$ & $95.98 \pm 1.11$ & $107.56 \pm 2.46$ & $103.70 \pm 1.74$ & $2069 \pm 23$ & 136.36 \\
\hline & $22487-04 \mathrm{G}$ & 25 & $0.04 \pm 0.08$ & $3.84 \pm 0.20$ & $64.81 \pm 1.82$ & $69.51 \pm 2.31$ & $68.65 \pm 1.64$ & $2046 \pm 44$ & 133.83 \\
\hline & $22487-04 \mathrm{H}$ & 26 & $0.05 \pm 0.08$ & $2.57 \pm 0.18$ & $39.90 \pm 0.66$ & $43.94 \pm 2.27$ & $42.89 \pm 1.61$ & $2064 \pm 49$ & 135.80 \\
\hline & $22487-04 \mathrm{I}$ & 27 & $0.06 \pm 0.10$ & $1.39 \pm 0.22$ & $19.90 \pm 1.27$ & $21.68 \pm 3.12$ & $20.36 \pm 2.21$ & $2000 \pm 150$ & 128.86 \\
\hline & $22487-04 \mathrm{~J}$ & 28 & $0.15 \pm 0.07$ & $2.14 \pm 0.19$ & $31.85 \pm 0.80$ & $38.47 \pm 2.23$ & $35.32 \pm 1.58$ & $2102 \pm 64$ & 140.05 \\
\hline & $22487-04 \mathrm{~K}$ & 29 & $0.18 \pm 0.07$ & $0.53 \pm 0.16$ & $5.32 \pm 0.99$ & $9.99 \pm 2.21$ & $6.14 \pm 1.56$ & $2150 \pm 400$ & 145.53 \\
\hline & $22487-04 \mathrm{~L}$ & 31 & $0.13 \pm 0.07$ & $1.13 \pm 0.16$ & $16.65 \pm 0.99$ & $20.92 \pm 2.08$ & $18.14 \pm 1.47$ & $2080 \pm 120$ & 137.58 \\
\hline B14-024_01 & $22489-01 \mathrm{~A}$ & 7 & $1.56 \pm 0.12$ & $12.50 \pm 0.27$ & $105.70 \pm 1.99$ & $83.27 \pm 0.82$ & $78.81 \pm 0.74$ & $1639 \pm 22$ & 93.98 \\
\hline$J=1.98570 \mathrm{e}-2$ & 22489-01B & 10 & $0.73 \pm 0.19$ & $24.48 \pm 0.25$ & $296.75 \pm 1.86$ & $319.34 \pm 1.34$ & $317.26 \pm 1.23$ & $2054.3 \pm 9.1$ & 134.74 \\
\hline \multirow[t]{8}{*}{ $\pm 3.99183 \mathrm{e}-5$} & $22489-01 C$ & 12 & $0.19 \pm 0.14$ & $17.65 \pm 0.23$ & $235.91 \pm 4.02$ & $253.22 \pm 0.61$ & $252.69 \pm 0.46$ & $2057 \pm 21$ & 135.03 \\
\hline & 22489-01D & 14 & $0.10 \pm 0.11$ & $15.98 \pm 0.20$ & $221.69 \pm 2.56$ & $238.52 \pm 0.54$ & $238.21 \pm 0.43$ & $2060 \pm 14$ & 135.36 \\
\hline & 22489-01E & 16 & $0.44 \pm 0.10$ & $11.59 \pm 0.20$ & $149.69 \pm 2.38$ & $160.26 \pm 0.50$ & $158.99 \pm 0.41$ & $2046 \pm 20$ & 133.83 \\
\hline & 22489-01F & 18 & & $5.87 \pm 0.12$ & $89.89 \pm 2.51$ & $95.69 \pm 0.80$ & $95.93 \pm 0.73$ & $2052 \pm 36$ & 134.48 \\
\hline & $22489-01 \mathrm{G}$ & 22 & $0.49 \pm 0.15$ & $9.98 \pm 0.22$ & $133.30 \pm 1.02$ & $144.10 \pm 1.60$ & $142.70 \pm 1.54$ & $2056 \pm 15$ & 134.92 \\
\hline & $22489-01 \mathrm{H}$ & 25 & & $3.92 \pm 0.16$ & $58.05 \pm 1.10$ & $60.96 \pm 0.50$ & $61.94 \pm 0.38$ & $2052 \pm 24$ & 134.48 \\
\hline & 22489-01I & 30 & $0.48 \pm 0.11$ & $4.92 \pm 0.17$ & $64.26 \pm 0.39$ & $70.00 \pm 0.50$ & $68.62 \pm 0.38$ & $2053.0 \pm 8.9$ & 134.59 \\
\hline & $22489-01 \mathrm{~J}$ & 35 & $1.32 \pm 0.12$ & $3.16 \pm 0.14$ & $38.43 \pm 0.28$ & $44.89 \pm 0.63$ & $41.12 \pm 0.54$ & $2055 \pm 18$ & 134.81 \\
\hline B14-024_02 & 22489-02A & 1.5 & $0.05 \pm 0.11$ & $0.01 \pm 0.15$ & & $0.14 \pm 1.77$ & $-0.49 \pm 1.25$ & $3900 \pm 7900$ & 487.89 \\
\hline
\end{tabular}




\begin{tabular}{|c|c|c|c|c|c|c|c|c|c|c|}
\hline$J=1.98570 \mathrm{e}-2$ & 22489-02B & 3 & $0.35 \pm 0.08$ & $0.40 \pm 0.13$ & $1.37 \pm 0.51$ & $0.83 \pm 1.36$ & & $\mathrm{~N} / \mathrm{A}$ & & \\
\hline \multirow[t]{20}{*}{ $\pm 3.99183 \mathrm{e}-5$} & $22489-02 \mathrm{C}$ & 5 & $0.65 \pm 0.10$ & $5.83 \pm 0.20$ & $53.01 \pm 0.62$ & $7.57 \pm 1.55$ & $0.17 \pm 1.10$ & $11 \pm 74$ & 0.39 & \\
\hline & 22489-02D & 7 & $2.45 \pm 0.11$ & $10.07 \pm 0.24$ & $117.30 \pm 0.99$ & $96.38 \pm 1.80$ & $68.47 \pm 1.28$ & $1389 \pm 20$ & 73.60 & \\
\hline & $22489-02 \mathrm{E}$ & 8 & $0.23 \pm 0.15$ & $6.32 \pm 0.25$ & $75.70 \pm 0.71$ & $82.65 \pm 2.37$ & $80.08 \pm 1.68$ & $2041 \pm 27$ & 133.28 & $\mathrm{x}$ \\
\hline & $22489-02 \mathrm{~F}$ & 9 & $0.41 \pm 0.10$ & $6.10 \pm 0.22$ & $72.56 \pm 0.78$ & $82.35 \pm 1.67$ & $77.65 \pm 1.18$ & $2056 \pm 22$ & 134.92 & $\mathrm{x}$ \\
\hline & $22489-02 \mathrm{G}$ & 10 & $0.43 \pm 0.10$ & $6.16 \pm 0.24$ & $76.15 \pm 0.79$ & $86.58 \pm 1.61$ & $81.68 \pm 1.15$ & $2059 \pm 21$ & 135.25 & $\mathrm{x}$ \\
\hline & 22489-02H & 11 & $0.59 \pm 0.23$ & $5.58 \pm 0.30$ & $70.04 \pm 0.73$ & $81.97 \pm 3.76$ & $75.23 \pm 2.66$ & $2060 \pm 45$ & 135.36 & $\mathrm{x}$ \\
\hline & 22489-02I & 12 & $0.47 \pm 0.23$ & $5.63 \pm 0.28$ & $70.93 \pm 0.75$ & $81.82 \pm 3.63$ & $76.50 \pm 2.57$ & $2065 \pm 43$ & 135.91 & $\mathrm{x}$ \\
\hline & 22489-02J & 13 & $0.33 \pm 0.14$ & $4.68 \pm 0.21$ & $59.86 \pm 1.07$ & $67.84 \pm 2.29$ & $64.11 \pm 1.63$ & $2057 \pm 38$ & 135.03 & $\mathrm{x}$ \\
\hline & $22489-02 \mathrm{~K}$ & 14 & $0.55 \pm 0.25$ & $14.17 \pm 0.38$ & $198.20 \pm 3.26$ & $218.41 \pm 4.11$ & $212.10 \pm 2.91$ & $2055 \pm 26$ & 134.81 & $\mathrm{x}$ \\
\hline & 22489-02L & 15 & $0.10 \pm 0.10$ & $4.01 \pm 0.19$ & $59.61 \pm 1.03$ & $65.89 \pm 1.58$ & $64.78 \pm 1.12$ & $2075 \pm 29$ & 137.02 & $\mathrm{x}$ \\
\hline & 22489-02M & 16 & $0.22 \pm 0.20$ & $1.63 \pm 0.25$ & $23.46 \pm 0.53$ & $26.44 \pm 3.29$ & $23.89 \pm 2.32$ & $2000 \pm 120$ & 128.86 & $\mathrm{x}$ \\
\hline & $22489-02 \mathrm{~N}$ & 17 & $0.40 \pm 0.12$ & $0.93 \pm 0.16$ & $9.46 \pm 0.75$ & $15.09 \pm 1.87$ & $10.58 \pm 1.32$ & $2110 \pm 180$ & 140.95 & $\mathrm{x}$ \\
\hline & $22489-02 \mathrm{O}$ & 18 & $0.29 \pm 0.11$ & $1.09 \pm 0.16$ & $16.58 \pm 0.57$ & $20.38 \pm 1.84$ & $17.12 \pm 1.30$ & $2010 \pm 100$ & 129.93 & $\mathrm{x}$ \\
\hline & 22489-02P & 19 & $0.17 \pm 0.15$ & $0.77 \pm 0.19$ & $11.58 \pm 0.35$ & $13.38 \pm 2.35$ & $11.41 \pm 1.66$ & $1960 \pm 180$ & 124.64 & $\mathrm{x}$ \\
\hline & 22489-02Q & 21 & $0.10 \pm 0.13$ & $0.91 \pm 0.17$ & $14.19 \pm 0.37$ & $16.47 \pm 2.04$ & $15.38 \pm 1.45$ & $2070 \pm 120$ & 136.47 & $\mathrm{x}$ \\
\hline & $22489-02 R$ & 23 & $0.17 \pm 0.08$ & $0.16 \pm 0.13$ & $2.54 \pm 0.87$ & $5.03 \pm 1.33$ & $3.09 \pm 0.94$ & $2220 \pm 590$ & 153.80 & $\mathrm{x}$ \\
\hline & $22489-02 S$ & 25 & $0.19 \pm 0.08$ & $0.02 \pm 0.12$ & & $1.43 \pm 1.30$ & $-0.73 \pm 0.92$ & $5200 \pm 9100$ & 1069.93 & $\mathrm{x}$ \\
\hline & $22489-02 \mathrm{~T}$ & 27 & $0.04 \pm 0.09$ & $0.22 \pm 0.13$ & $3.50 \pm 0.55$ & $4.33 \pm 1.43$ & $3.82 \pm 1.01$ & $2080 \pm 380$ & 137.58 & $\mathrm{x}$ \\
\hline & $22489-02 \mathrm{U}$ & 29 & $0.03 \pm 0.08$ & $0.07 \pm 0.12$ & & $0.05 \pm 1.30$ & $-0.25 \pm 0.92$ & $4000 \pm 14000$ & 519.31 & $\mathrm{x}$ \\
\hline & $22489-02 \mathrm{~V}$ & 33 & & $0.13 \pm 0.16$ & $1.45 \pm 0.39$ & $1.50 \pm 2.00$ & $2.64 \pm 1.42$ & $2760 \pm 850$ & 229.62 & $\mathrm{x}$ \\
\hline B14-045_01 & $22490-02 \mathrm{~A}$ & 3 & $0.50 \pm 0.09$ & $3.35 \pm 0.13$ & $45.23 \pm 0.42$ & $25.32 \pm 0.55$ & $23.16 \pm 0.39$ & $1266 \pm 18$ & 64.57 & \\
\hline$J=1.98722 \mathrm{e}-2$ & $22490-02 B$ & 5 & $0.45 \pm 0.10$ & $10.43 \pm 0.19$ & $162.40 \pm 0.69$ & $124.76 \pm 0.62$ & $122.80 \pm 0.44$ & $1654.5 \pm 6.2$ & 95.34 & \\
\hline \multirow[t]{5}{*}{ $\pm 2.50624 \mathrm{e}-5$} & $22490-02 \mathrm{C}$ & 6.5 & & $14.44 \pm 0.24$ & $206.30 \pm 1.00$ & $211.15 \pm 0.75$ & $211.20 \pm 0.54$ & $2002.8 \pm 6.8$ & 129.16 & \\
\hline & $22490-02 \mathrm{D}$ & 7 & $0.15 \pm 0.09$ & $13.00 \pm 0.20$ & $180.30 \pm 0.76$ & $193.17 \pm 0.53$ & $192.50 \pm 0.38$ & $2053.2 \pm 5.9$ & 134.61 & $\mathrm{x}$ \\
\hline & $22490-02 \mathrm{E}$ & 7.5 & & $11.68 \pm 0.19$ & $165.10 \pm 1.25$ & $177.33 \pm 0.51$ & $177.40 \pm 0.37$ & $2061.5 \pm 9.8$ & 135.53 & $\mathrm{x}$ \\
\hline & $22490-02 \mathrm{~F}$ & 8 & $0.05 \pm 0.08$ & $10.83 \pm 0.18$ & $152.20 \pm 1.14$ & $163.50 \pm 0.52$ & $163.30 \pm 0.38$ & $2059.8 \pm 9.7$ & 135.34 & $\mathrm{x}$ \\
\hline & $22490-02 \mathrm{G}$ & 11 & $0.07 \pm 0.16$ & $33.80 \pm 0.29$ & $500.94 \pm 6.09$ & $532.46 \pm 1.07$ & $532.14 \pm 0.75$ & $2048 \pm 15$ & 134.04 & $\mathrm{x}$ \\
\hline
\end{tabular}




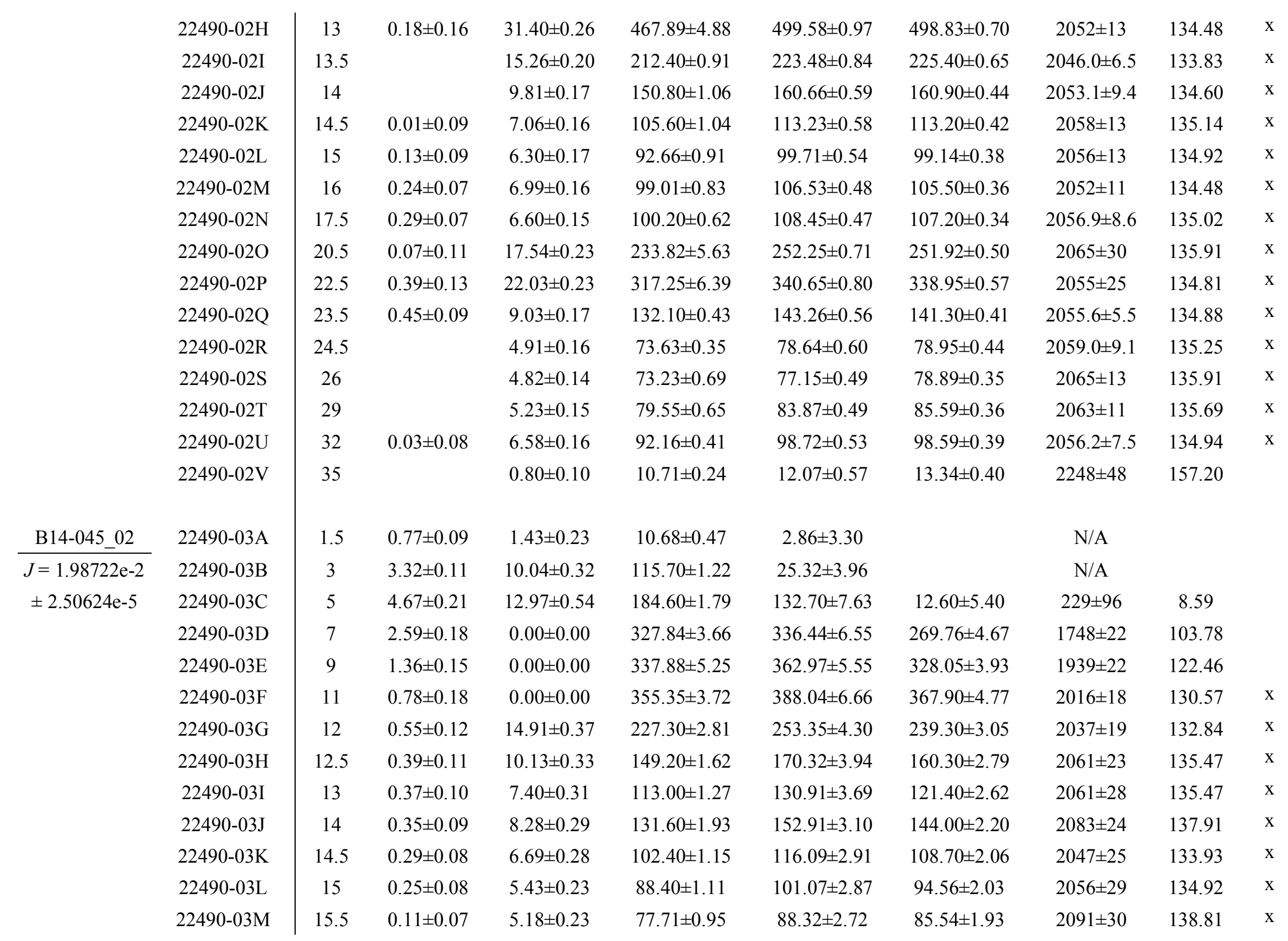




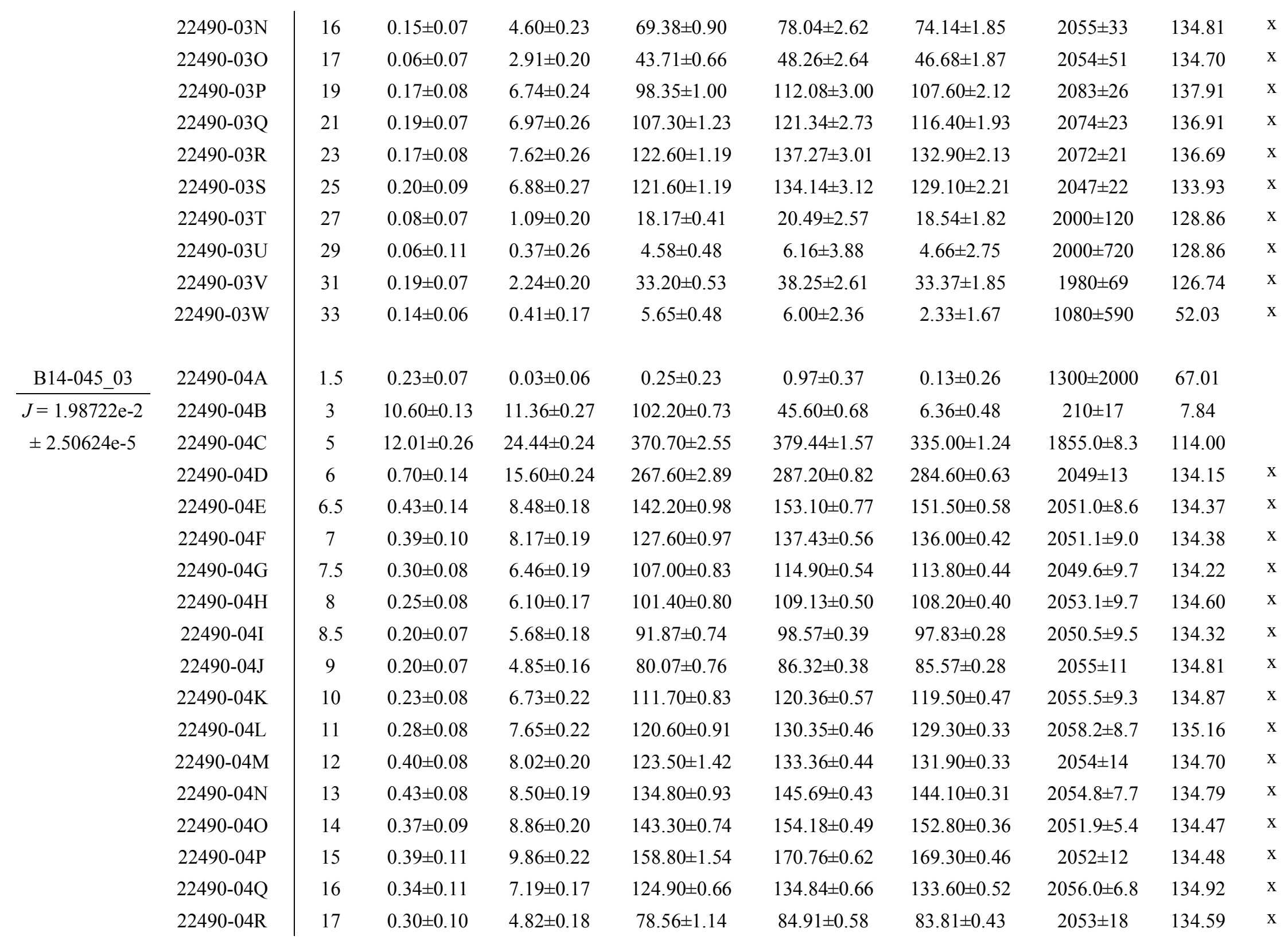




\begin{tabular}{|c|c|c|c|c|c|c|c|c|c|}
\hline & 22490-04S & 18 & $0.21 \pm 0.08$ & $5.03 \pm 0.17$ & $87.45 \pm 0.68$ & $95.03 \pm 0.42$ & $94.27 \pm 0.31$ & $2065.6 \pm 9.4$ & 135.98 \\
\hline & $22490-04 \mathrm{~T}$ & 19 & $0.03 \pm 0.07$ & $4.59 \pm 0.16$ & $79.53 \pm 0.73$ & $84.88 \pm 0.38$ & $84.76 \pm 0.28$ & $2051 \pm 11$ & 134.37 \\
\hline & $22490-04 \mathrm{U}$ & 20 & & $1.10 \pm 0.10$ & $18.47 \pm 0.38$ & $19.77 \pm 0.35$ & $19.82 \pm 0.25$ & $2060 \pm 29$ & 135.36 \\
\hline & $22490-04 \mathrm{~V}$ & 23 & $0.03 \pm 0.07$ & $0.89 \pm 0.08$ & $13.35 \pm 0.38$ & $14.39 \pm 0.36$ & $14.26 \pm 0.26$ & $2054 \pm 41$ & 134.70 \\
\hline & 22490-04W & 26 & $0.12 \pm 0.07$ & $0.19 \pm 0.07$ & $3.24 \pm 0.26$ & $3.99 \pm 0.37$ & $3.56 \pm 0.27$ & $2090 \pm 140$ & 138.70 \\
\hline & $22490-04 X$ & 29 & $0.11 \pm 0.07$ & $0.03 \pm 0.07$ & $1.25 \pm 0.25$ & $1.59 \pm 0.37$ & $1.18 \pm 0.27$ & $1910 \pm 350$ & 119.50 \\
\hline & $22490-04 Y$ & 32 & $0.24 \pm 0.07$ & $1.89 \pm 0.11$ & $32.65 \pm 0.44$ & $35.61 \pm 0.39$ & $34.72 \pm 0.29$ & $2049 \pm 19$ & 134.15 \\
\hline B14-049_01 & 22488-01A & 7 & $3.75 \pm 0.24$ & $68.09 \pm 0.48$ & $772.06 \pm 9.94$ & $762.30 \pm 1.04$ & $751.78 \pm 0.80$ & $1943 \pm 15$ & 122.88 \\
\hline$J=1.98827 \mathrm{e}-2$ & 22488-01B & 8 & $0.66 \pm 0.14$ & $18.18 \pm 0.25$ & $233.20 \pm 3.40$ & $238.45 \pm 0.82$ & $236.60 \pm 0.73$ & $1992.5 \pm 8.4$ & 128.06 \\
\hline \multirow[t]{10}{*}{ $\pm 2.50624 \mathrm{e}-5$} & 22488-01C & 10 & $0.89 \pm 0.15$ & $34.10 \pm 0.32$ & $402.15 \pm 5.89$ & $430.93 \pm 0.63$ & $428.43 \pm 0.48$ & $2052 \pm 18$ & 134.48 \\
\hline & 22488-01D & 12 & $0.49 \pm 0.21$ & $40.38 \pm 0.36$ & $631.56 \pm 6.73$ & $674.33 \pm 1.19$ & $672.98 \pm 1.04$ & $2052 \pm 13$ & 134.48 \\
\hline & $22488-01 \mathrm{E}$ & 14 & $0.80 \pm 0.23$ & $47.50 \pm 0.32$ & $768.29 \pm 10.32$ & $827.79 \pm 1.20$ & $825.56 \pm 1.00$ & $2062 \pm 17$ & 135.58 \\
\hline & $22488-01 \mathrm{~F}$ & 16 & $1.68 \pm 0.24$ & $43.63 \pm 0.39$ & $695.83 \pm 7.18$ & $748.45 \pm 0.98$ & $743.74 \pm 0.72$ & $2056 \pm 13$ & 134.92 \\
\hline & $22488-01 G$ & 18 & $1.33 \pm 0.18$ & $35.80 \pm 0.39$ & $548.10 \pm 6.25$ & $587.08 \pm 0.77$ & $583.38 \pm 0.58$ & $2050 \pm 14$ & 134.26 \\
\hline & 22488-01H & 20 & $1.45 \pm 0.14$ & $28.23 \pm 0.27$ & $355.87 \pm 4.67$ & $383.84 \pm 0.66$ & $379.81 \pm 0.53$ & $2054 \pm 16$ & 134.70 \\
\hline & 22488-01I & 23 & $1.83 \pm 0.13$ & $25.58 \pm 0.29$ & $325.10 \pm 4.67$ & $352.81 \pm 0.63$ & $347.70 \pm 0.51$ & $2056 \pm 18$ & 134.92 \\
\hline & $22488-01 \mathrm{~J}$ & 26 & $5.13 \pm 0.11$ & $5.95 \pm 0.17$ & $67.00 \pm 1.31$ & $76.82 \pm 0.47$ & $62.46 \pm 0.35$ & $1892 \pm 20$ & 117.68 \\
\hline & $22488-01 K$ & 30 & $1.31 \pm 0.13$ & $2.24 \pm 0.11$ & $25.08 \pm 0.52$ & $27.51 \pm 0.50$ & $23.84 \pm 0.35$ & $1915 \pm 26$ & 120.01 \\
\hline & 22488-01L & 35 & $0.87 \pm 0.10$ & $1.17 \pm 0.09$ & $10.81 \pm 0.49$ & $13.26 \pm 0.39$ & $10.83 \pm 0.28$ & $1976 \pm 61$ & 126.32 \\
\hline B14-049_02 & $22488-02 \mathrm{~A}$ & 1.5 & $0.70 \pm 0.13$ & $0.17 \pm 0.10$ & $0.43 \pm 0.38$ & $2.47 \pm 0.51$ & $0.61 \pm 0.36$ & $2400 \pm 1400$ & 176.60 \\
\hline$J=1.98827 \mathrm{e}-2$ & 22488-02B & 3 & $0.08 \pm 0.13$ & $0.01 \pm 0.10$ & $0.51 \pm 0.38$ & $0.48 \pm 0.49$ & $0.25 \pm 0.35$ & $1200 \pm 1400$ & 59.97 \\
\hline \multirow[t]{6}{*}{ $\pm 2.50624 \mathrm{e}-5$} & $22488-02 \mathrm{C}$ & 5 & $0.14 \pm 0.09$ & $0.01 \pm 0.10$ & $0.56 \pm 0.37$ & $0.62 \pm 0.34$ & $0.26 \pm 0.24$ & $1170 \pm 980$ & 57.93 \\
\hline & 22488-02D & 7 & $0.26 \pm 0.09$ & $0.10 \pm 0.10$ & $1.10 \pm 0.25$ & $0.29 \pm 0.34$ & & N/A & \\
\hline & $22488-02 \mathrm{E}$ & 1.5 & & & & $0.23 \pm 0.31$ & $0.59 \pm 0.22$ & N/A & \\
\hline & $22488-02 \mathrm{~F}$ & 3 & & & & $0.19 \pm 0.31$ & $0.36 \pm 0.22$ & N/A & \\
\hline & $22488-02 \mathrm{G}$ & 7 & & $0.16 \pm 0.10$ & $0.22 \pm 0.41$ & $0.15 \pm 0.34$ & $0.44 \pm 0.24$ & $2900 \pm 2800$ & 253.27 \\
\hline & 22488-02H & 9 & $0.62 \pm 0.13$ & $2.11 \pm 0.15$ & $15.92 \pm 0.40$ & $3.21 \pm 0.48$ & $1.57 \pm 0.34$ & $322 \pm 65$ & 12.40 \\
\hline
\end{tabular}




\begin{tabular}{|c|c|c|c|c|c|c|c|c|c|}
\hline & 22488-02I & 11 & $0.85 \pm 0.17$ & $12.39 \pm 0.23$ & $125.10 \pm 0.79$ & $110.07 \pm 0.65$ & $107.80 \pm 0.46$ & $1800.3 \pm 8.4$ & 108.68 \\
\hline & $22488-02 \mathrm{~J}$ & 12 & $0.40 \pm 0.13$ & $11.03 \pm 0.22$ & $118.90 \pm 0.78$ & $128.17 \pm 0.50$ & $127.10 \pm 0.36$ & $2056.1 \pm 8.5$ & 134.93 \\
\hline & $22488-02 \mathrm{~K}$ & 13 & & $7.29 \pm 0.20$ & $84.38 \pm 1.25$ & $89.89 \pm 0.38$ & $90.12 \pm 0.28$ & $2055 \pm 18$ & 134.81 \\
\hline & $22488-02 \mathrm{~L}$ & 14 & $0.22 \pm 0.10$ & $6.31 \pm 0.20$ & $73.67 \pm 0.96$ & $79.44 \pm 0.39$ & $78.86 \pm 0.28$ & $2058 \pm 16$ & 135.14 \\
\hline & $22488-02 \mathrm{M}$ & 16 & $0.13 \pm 0.11$ & $11.81 \pm 0.24$ & $129.20 \pm 0.85$ & $139.35 \pm 0.44$ & $139.00 \pm 0.32$ & $2064.3 \pm 8.2$ & 135.84 \\
\hline & $22488-02 \mathrm{~N}$ & 18 & $0.24 \pm 0.11$ & $13.69 \pm 0.28$ & $158.50 \pm 1.55$ & $170.25 \pm 0.49$ & $169.60 \pm 0.39$ & $2057 \pm 12$ & 135.03 \\
\hline & $22488-02 \mathrm{O}$ & 19 & $0.22 \pm 0.10$ & $6.98 \pm 0.22$ & $86.90 \pm 1.08$ & $93.53 \pm 0.39$ & $92.94 \pm 0.28$ & $2056 \pm 16$ & 134.92 \\
\hline & $22488-02 \mathrm{P}$ & 20 & $0.24 \pm 0.10$ & $5.43 \pm 0.17$ & $66.61 \pm 0.63$ & $71.97 \pm 0.38$ & $71.34 \pm 0.28$ & $2058 \pm 12$ & 135.14 \\
\hline & 22488-02Q & 21 & $0.28 \pm 0.11$ & $11.64 \pm 0.26$ & $145.10 \pm 0.60$ & $156.06 \pm 0.45$ & $155.30 \pm 0.34$ & $2057.5 \pm 5.2$ & 135.09 \\
\hline & $22488-02 R$ & 22 & $0.19 \pm 0.09$ & $4.31 \pm 0.16$ & $51.41 \pm 0.58$ & $54.67 \pm 0.37$ & $54.16 \pm 0.27$ & $2038 \pm 15$ & 132.95 \\
\hline & $22488-02 S$ & 23 & $0.15 \pm 0.10$ & $4.05 \pm 0.14$ & $54.80 \pm 0.60$ & $58.74 \pm 0.40$ & $58.33 \pm 0.29$ & $2051 \pm 15$ & 134.37 \\
\hline & $22488-02 \mathrm{~T}$ & 24 & $0.26 \pm 0.09$ & $4.46 \pm 0.16$ & $56.33 \pm 0.55$ & $60.79 \pm 0.35$ & $60.10 \pm 0.25$ & $2053 \pm 13$ & 134.59 \\
\hline & $22488-02 \mathrm{U}$ & 25 & $0.00 \pm 0.10$ & $2.85 \pm 0.14$ & $34.41 \pm 0.49$ & $36.54 \pm 0.38$ & $36.53 \pm 0.27$ & $2047 \pm 19$ & 133.93 \\
\hline & $22488-02 \mathrm{~V}$ & 26 & $0.07 \pm 0.09$ & $1.01 \pm 0.11$ & $10.74 \pm 0.34$ & $11.86 \pm 0.35$ & $11.67 \pm 0.25$ & $2076 \pm 47$ & 137.13 \\
\hline & $22488-02 W$ & 27 & $0.12 \pm 0.09$ & $2.52 \pm 0.14$ & $32.88 \pm 0.47$ & $35.26 \pm 0.35$ & $34.95 \pm 0.25$ & $2049 \pm 20$ & 134.15 \\
\hline & $22488-02 X$ & 29 & $0.07 \pm 0.08$ & $0.47 \pm 0.11$ & $8.20 \pm 0.41$ & $8.19 \pm 0.31$ & $8.01 \pm 0.22$ & $1946 \pm 68$ & 123.19 \\
\hline & $22488-02 Y$ & 31 & $0.18 \pm 0.08$ & $0.53 \pm 0.11$ & $7.35 \pm 0.57$ & $8.12 \pm 0.31$ & $7.64 \pm 0.22$ & $2020 \pm 100$ & 131.00 \\
\hline & $22488-02 Z$ & 33 & $0.24 \pm 0.08$ & $0.43 \pm 0.11$ & $4.95 \pm 0.43$ & $6.11 \pm 0.32$ & $5.49 \pm 0.23$ & $2100 \pm 120$ & 139.82 \\
\hline B14-049_03 & 22488-03A & 1.5 & $0.29 \pm 0.08$ & $0.67 \pm 0.11$ & $4.80 \pm 0.23$ & $0.93 \pm 0.67$ & $-0.83 \pm 0.47$ & $\mathrm{~N} / \mathrm{A}$ & \\
\hline$J=1.98827 \mathrm{e}-2$ & 22488-03B & 3 & $0.61 \pm 0.09$ & $8.28 \pm 0.19$ & $63.40 \pm 0.46$ & $10.78 \pm 0.75$ & $7.09 \pm 0.53$ & $362 \pm 25$ & 14.10 \\
\hline $\pm 2.50624 \mathrm{e}-5$ & $22488-03 \mathrm{C}$ & 5 & $1.56 \pm 0.15$ & $33.08 \pm 0.34$ & $317.80 \pm 1.10$ & $221.85 \pm 1.32$ & $212.50 \pm 0.95$ & $1525.7 \pm 6.0$ & 84.39 \\
\hline & 22488-03D & 7 & $6.27 \pm 0.52$ & $50.13 \pm 0.30$ & $436.89 \pm 2.14$ & $449.45 \pm 4.49$ & $411.88 \pm 3.21$ & $1905 \pm 11$ & 118.99 \\
\hline & $22488-03 E$ & 9 & $0.77 \pm 0.22$ & $50.86 \pm 0.34$ & $512.33 \pm 2.17$ & $556.14 \pm 1.94$ & $551.52 \pm 1.40$ & $2064.5 \pm 6.2$ & 135.86 \\
\hline & $22488-03 \mathrm{~F}$ & 10 & $0.26 \pm 0.17$ & $28.30 \pm 0.25$ & $303.91 \pm 2.09$ & $338.62 \pm 1.42$ & $337.06 \pm 1.01$ & $2101.3 \pm 9.4$ & 139.97 \\
\hline & $22488-03 G$ & 11 & $0.34 \pm 0.13$ & $24.65 \pm 0.29$ & $252.30 \pm 2.75$ & $292.67 \pm 1.21$ & $290.60 \pm 0.93$ & $2148 \pm 14$ & 145.30 \\
\hline & $22488-03 \mathrm{H}$ & 12 & $0.19 \pm 0.19$ & $27.73 \pm 0.28$ & $294.60 \pm 2.09$ & $333.84 \pm 1.60$ & $332.70 \pm 1.15$ & $2123.6 \pm 9.9$ & 142.50 \\
\hline & 22488-03I & 12.5 & $0.19 \pm 0.14$ & $16.85 \pm 0.30$ & $178.80 \pm 0.89$ & $205.36 \pm 1.17$ & $204.20 \pm 0.84$ & $2137.5 \pm 8.1$ & 144.09 \\
\hline & $22488-03 \mathrm{~J}$ & 13 & $0.43 \pm 0.14$ & $13.88 \pm 0.29$ & $148.00 \pm 1.60$ & $162.96 \pm 1.16$ & $160.40 \pm 0.83$ & $2073 \pm 15$ & 136.80 \\
\hline
\end{tabular}




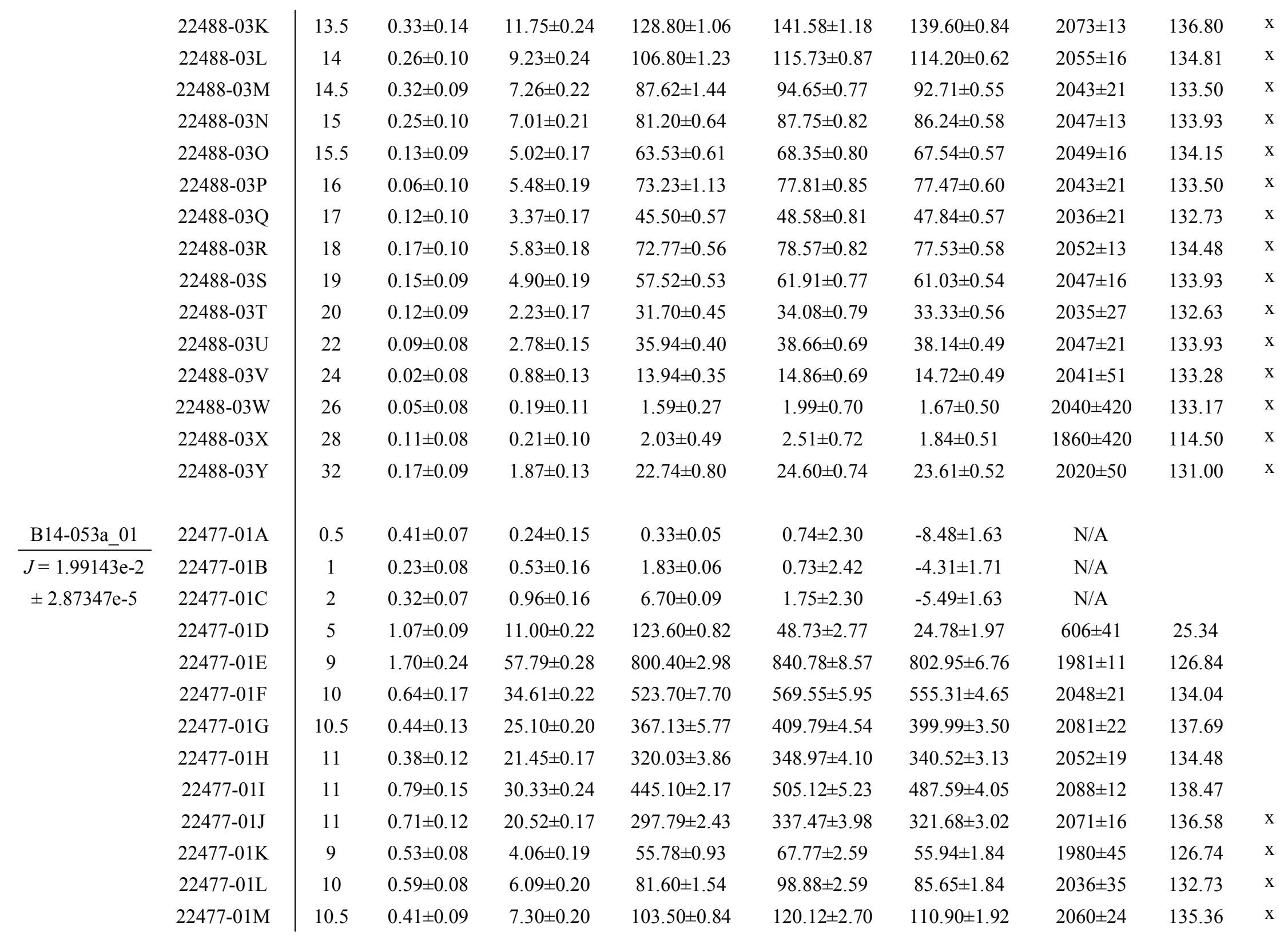




\begin{tabular}{|c|c|c|c|c|c|c|c|c|}
\hline $22477-01 \mathrm{~N}$ & 11 & $0.34 \pm 0.08$ & $8.79 \pm 0.23$ & $127.10 \pm 0.74$ & $142.33 \pm 2.66$ & $134.80 \pm 1.89$ & $2048 \pm 19$ & 134.04 \\
\hline $22477-010$ & 11.2 & $0.36 \pm 0.08$ & $8.08 \pm 0.21$ & $114.30 \pm 0.76$ & $128.80 \pm 2.68$ & $120.80 \pm 1.90$ & $2044 \pm 21$ & 133.61 \\
\hline $22477-01 P$ & 11.3 & $0.26 \pm 0.08$ & $7.05 \pm 0.21$ & $106.50 \pm 1.92$ & $118.79 \pm 2.65$ & $113.10 \pm 1.89$ & $2050 \pm 30$ & 134.26 \\
\hline 22477-01Q & 11.4 & $0.21 \pm 0.08$ & $6.68 \pm 0.21$ & $97.93 \pm 2.06$ & $110.54 \pm 2.47$ & $105.80 \pm 1.77$ & $2071 \pm 33$ & 136.58 \\
\hline 22477-01R & 11.5 & $0.26 \pm 0.08$ & $5.61 \pm 0.19$ & $84.09 \pm 0.67$ & $95.25 \pm 2.47$ & $89.55 \pm 1.76$ & $2053 \pm 26$ & 134.59 \\
\hline $22477-01 \mathrm{~S}$ & 11.6 & $0.22 \pm 0.08$ & $5.26 \pm 0.19$ & $77.41 \pm 0.64$ & $87.58 \pm 2.51$ & $82.74 \pm 1.78$ & $2058 \pm 29$ & 135.14 \\
\hline $22477-01 \mathrm{~T}$ & 11.8 & $0.15 \pm 0.08$ & $5.20 \pm 0.18$ & $77.56 \pm 1.37$ & $87.48 \pm 2.45$ & $84.03 \pm 1.77$ & $2074 \pm 34$ & 136.91 \\
\hline $22477-01 \mathrm{U}$ & 12.1 & $0.07 \pm 0.08$ & $5.51 \pm 0.19$ & $80.27 \pm 1.16$ & $89.76 \pm 2.53$ & $88.29 \pm 1.80$ & $2093 \pm 31$ & 139.03 \\
\hline $22477-01 \mathrm{~V}$ & 12.4 & $0.01 \pm 0.08$ & $5.65 \pm 0.20$ & $83.29 \pm 0.91$ & $90.56 \pm 2.47$ & $90.36 \pm 1.75$ & $2076 \pm 28$ & 137.13 \\
\hline $22477-01 \mathrm{~W}$ & 12.8 & $0.14 \pm 0.08$ & $6.37 \pm 0.19$ & $88.90 \pm 0.62$ & $98.80 \pm 2.49$ & $95.61 \pm 1.77$ & $2065 \pm 24$ & 135.91 \\
\hline 22477-01X & 13.5 & $0.21 \pm 0.09$ & $8.51 \pm 0.22$ & $118.50 \pm 1.38$ & $134.21 \pm 2.76$ & $129.60 \pm 1.96$ & $2085 \pm 24$ & 138.14 \\
\hline $22477-01 Y$ & 14 & $0.33 \pm 0.09$ & $9.02 \pm 0.21$ & $124.20 \pm 0.66$ & $138.86 \pm 2.77$ & $131.60 \pm 1.97$ & $2047 \pm 20$ & 133.93 \\
\hline $22477-01 Z$ & 14.5 & $0.40 \pm 0.12$ & $9.26 \pm 0.26$ & $130.30 \pm 0.74$ & $149.16 \pm 3.67$ & $140.30 \pm 2.62$ & $2066 \pm 24$ & 136.02 \\
\hline 22477-01AA & 15 & $0.20 \pm 0.12$ & $10.25 \pm 0.29$ & $144.70 \pm 1.41$ & $160.97 \pm 3.83$ & $156.40 \pm 2.72$ & $2071 \pm 25$ & 136.58 \\
\hline $22477-01 \mathrm{AB}$ & 16 & $0.44 \pm 0.10$ & $14.97 \pm 0.25$ & $211.20 \pm 1.57$ & $230.55 \pm 3.13$ & $220.70 \pm 2.26$ & $2030 \pm 16$ & 132.08 \\
\hline $22477-01 \mathrm{AC}$ & 17 & $0.63 \pm 0.11$ & $17.89 \pm 0.16$ & $253.56 \pm 1.14$ & $285.89 \pm 5.25$ & $271.76 \pm 4.68$ & $2061 \pm 22$ & 135.47 \\
\hline 22477-01AD & 17.5 & $0.49 \pm 0.10$ & $14.43 \pm 0.15$ & $218.46 \pm 1.97$ & $244.70 \pm 4.71$ & $233.71 \pm 4.18$ & $2059 \pm 25$ & 135.25 \\
\hline 22477-01AE & 18 & $0.34 \pm 0.09$ & $11.12 \pm 0.22$ & $164.80 \pm 0.92$ & $183.71 \pm 2.90$ & $176.20 \pm 2.08$ & $2058 \pm 16$ & 135.14 \\
\hline 22477-01AF & 18.5 & $0.30 \pm 0.09$ & $9.86 \pm 0.23$ & $144.60 \pm 1.50$ & $159.79 \pm 2.85$ & $153.20 \pm 2.03$ & $2047 \pm 21$ & 133.93 \\
\hline $22477-01 \mathrm{AG}$ & 19 & $0.28 \pm 0.12$ & $11.87 \pm 0.28$ & $182.00 \pm 1.08$ & $198.85 \pm 3.67$ & $192.70 \pm 2.60$ & $2047 \pm 18$ & 133.93 \\
\hline $22477-01 \mathrm{AH}$ & 19.5 & $0.26 \pm 0.08$ & $7.80 \pm 0.20$ & $123.00 \pm 0.79$ & $133.71 \pm 2.59$ & $127.80 \pm 1.83$ & $2023 \pm 19$ & 131.33 \\
\hline $22477-01 \mathrm{AI}$ & 20 & $0.13 \pm 0.08$ & $6.20 \pm 0.19$ & $93.04 \pm 0.57$ & $103.64 \pm 2.56$ & $100.80 \pm 1.82$ & $2075 \pm 14$ & 137.02 \\
\hline $22477-01 \mathrm{AJ}$ & 20.5 & $0.20 \pm 0.08$ & $6.68 \pm 0.20$ & $106.20 \pm 0.79$ & $116.30 \pm 2.60$ & $111.80 \pm 1.85$ & $2039 \pm 22$ & 133.06 \\
\hline 22477-01AK & 21 & $0.18 \pm 0.08$ & $5.95 \pm 0.19$ & $88.47 \pm 0.65$ & $98.92 \pm 2.48$ & $94.96 \pm 1.76$ & $2063 \pm 25$ & 135.69 \\
\hline 22477-01AL & 21.5 & $0.12 \pm 0.08$ & $3.96 \pm 0.18$ & $59.95 \pm 0.28$ & $68.35 \pm 2.42$ & $65.63 \pm 1.72$ & $2087 \pm 33$ & 138.36 \\
\hline 22477-01AM & 22 & $0.17 \pm 0.08$ & $2.48 \pm 0.17$ & $38.18 \pm 0.28$ & $43.95 \pm 2.45$ & $40.06 \pm 1.74$ & $2035 \pm 54$ & 132.63 \\
\hline $22477-01 \mathrm{AN}$ & 22.5 & $0.06 \pm 0.08$ & $0.91 \pm 0.16$ & $14.04 \pm 0.07$ & $16.07 \pm 2.42$ & $14.68 \pm 1.72$ & $2030 \pm 140$ & 132.08 \\
\hline $22477-01 \mathrm{AO}$ & 23 & $0.08 \pm 0.07$ & $1.00 \pm 0.16$ & $14.16 \pm 0.08$ & $16.23 \pm 2.36$ & $14.40 \pm 1.67$ & $2000 \pm 140$ & 128.86 \\
\hline 22477-01AP & 24 & & $0.53 \pm 0.16$ & $9.00 \pm 0.20$ & $9.26 \pm 2.36$ & $12.79 \pm 1.67$ & $2420 \pm 180$ & 179.28 \\
\hline
\end{tabular}




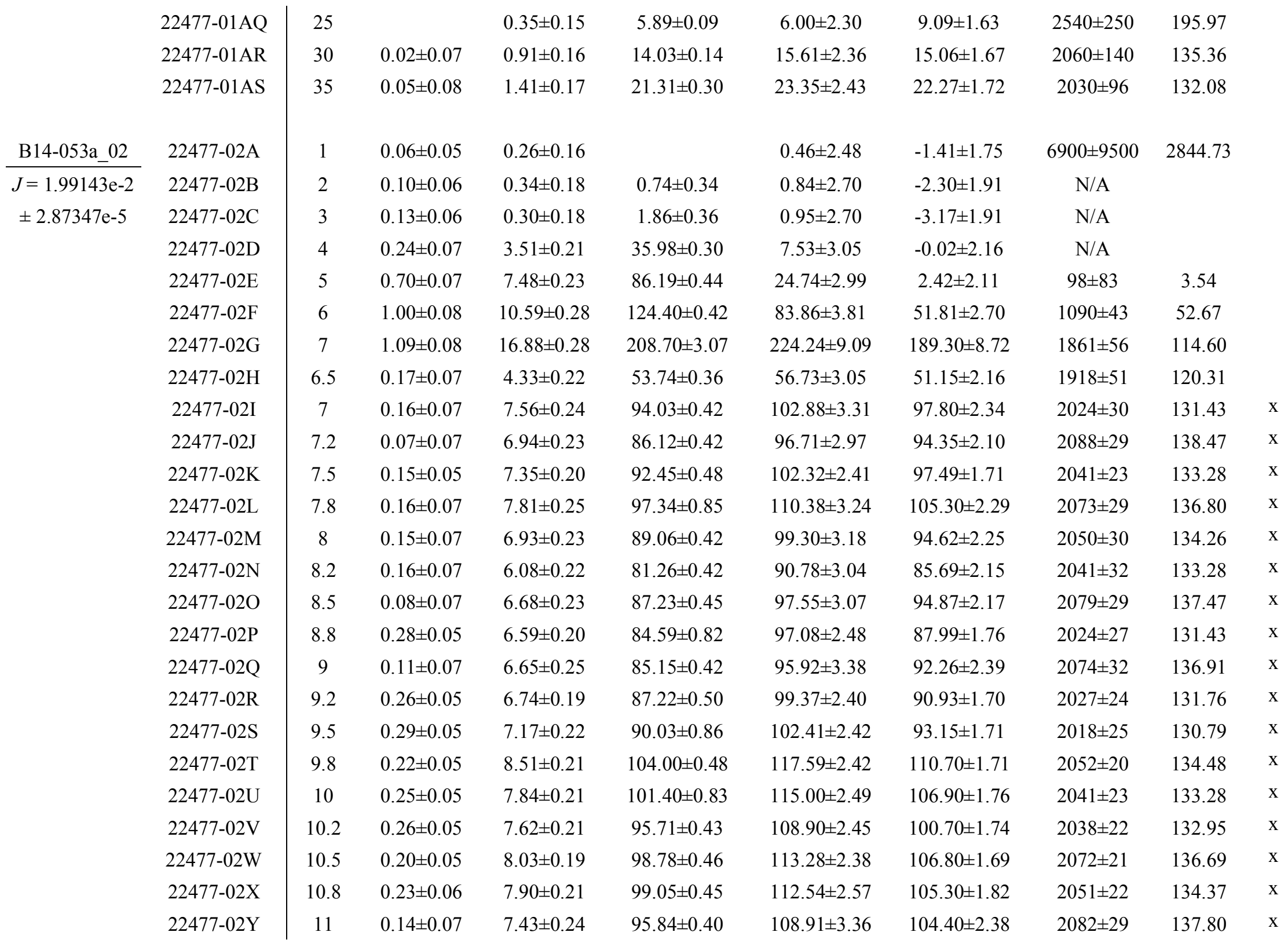




\begin{tabular}{|c|c|c|c|c|c|c|c|c|}
\hline $22477-02 Z$ & 11.2 & $0.17 \pm 0.05$ & $6.95 \pm 0.19$ & $87.21 \pm 0.40$ & $98.74 \pm 2.32$ & $93.28 \pm 1.64$ & $2059 \pm 23$ & 135.25 \\
\hline 22477-02AA & 11.5 & $0.24 \pm 0.05$ & $6.84 \pm 0.19$ & $87.89 \pm 0.42$ & $99.78 \pm 2.41$ & $91.96 \pm 1.71$ & $2032 \pm 24$ & 132.30 \\
\hline $22477-02 \mathrm{AB}$ & 11.8 & $0.12 \pm 0.07$ & $6.62 \pm 0.23$ & $86.74 \pm 0.45$ & $97.14 \pm 3.11$ & $93.36 \pm 2.20$ & $2066 \pm 30$ & 136.02 \\
\hline $22477-02 \mathrm{AC}$ & 12 & $0.14 \pm 0.07$ & $6.50 \pm 0.24$ & $85.38 \pm 0.47$ & $95.79 \pm 3.32$ & $91.24 \pm 2.35$ & $2057 \pm 33$ & 135.03 \\
\hline 22477-02AD & 12.2 & $0.04 \pm 0.07$ & $6.76 \pm 0.23$ & $85.46 \pm 0.40$ & $96.42 \pm 3.16$ & $95.19 \pm 2.24$ & $2109 \pm 30$ & 140.84 \\
\hline $22477-02 \mathrm{AE}$ & 12.5 & $0.11 \pm 0.07$ & $7.14 \pm 0.24$ & $87.71 \pm 0.37$ & $99.64 \pm 3.28$ & $96.21 \pm 2.32$ & $2090 \pm 31$ & 138.70 \\
\hline $22477-02 \mathrm{AF}$ & 12.8 & $0.20 \pm 0.05$ & $6.59 \pm 0.19$ & $82.91 \pm 0.42$ & $94.15 \pm 2.47$ & $87.67 \pm 1.75$ & $2044 \pm 25$ & 133.61 \\
\hline $22477-02 \mathrm{AG}$ & 13 & $0.11 \pm 0.07$ & $6.10 \pm 0.24$ & $80.21 \pm 0.42$ & $90.39 \pm 3.25$ & $86.90 \pm 2.30$ & $2074 \pm 33$ & 136.91 \\
\hline $22477-02 \mathrm{AH}$ & 13.3 & $0.06 \pm 0.07$ & $6.17 \pm 0.23$ & $77.75 \pm 0.45$ & $88.09 \pm 3.13$ & $86.13 \pm 2.22$ & $2102 \pm 33$ & 140.05 \\
\hline 22477-02AI & 13.7 & $0.26 \pm 0.05$ & $6.30 \pm 0.18$ & $81.84 \pm 0.42$ & $92.63 \pm 2.30$ & $84.24 \pm 1.63$ & $2012 \pm 25$ & 130.14 \\
\hline 22477-02AJ & 14 & $0.21 \pm 0.05$ & $6.95 \pm 0.20$ & $87.84 \pm 0.47$ & $99.79 \pm 2.34$ & $93.23 \pm 1.65$ & $2049 \pm 23$ & 134.15 \\
\hline 22477-02AK & 14.3 & $0.12 \pm 0.07$ & $7.17 \pm 0.22$ & $87.65 \pm 0.44$ & $98.23 \pm 3.01$ & $94.54 \pm 2.13$ & $2069 \pm 29$ & 136.36 \\
\hline $22477-02 \mathrm{AL}$ & 14.7 & $0.14 \pm 0.07$ & $7.23 \pm 0.25$ & $93.53 \pm 0.79$ & $105.06 \pm 3.36$ & $100.60 \pm 2.38$ & $2065 \pm 31$ & 135.91 \\
\hline 22477-02AM & 15 & $0.19 \pm 0.07$ & $6.91 \pm 0.22$ & $85.10 \pm 0.82$ & $96.54 \pm 3.02$ & $90.45 \pm 2.14$ & $2051 \pm 32$ & 134.37 \\
\hline $22477-02 \mathrm{AN}$ & 15.3 & $0.14 \pm 0.06$ & $6.68 \pm 0.23$ & $87.67 \pm 0.42$ & $99.22 \pm 2.91$ & $94.80 \pm 2.06$ & $2072 \pm 28$ & 136.69 \\
\hline $22477-02 \mathrm{AO}$ & 15.7 & $0.20 \pm 0.05$ & $6.89 \pm 0.19$ & $87.87 \pm 0.42$ & $100.67 \pm 2.18$ & $94.22 \pm 1.55$ & $2062 \pm 21$ & 135.58 \\
\hline 22477-02AP & 16 & $0.26 \pm 0.05$ & $6.86 \pm 0.18$ & $85.91 \pm 0.40$ & $97.73 \pm 2.17$ & $89.48 \pm 1.53$ & $2026 \pm 22$ & 131.65 \\
\hline 22477-02AQ & 16.3 & $0.21 \pm 0.05$ & $7.19 \pm 0.18$ & $87.82 \pm 0.40$ & $99.74 \pm 2.27$ & $93.13 \pm 1.61$ & $2048 \pm 22$ & 134.04 \\
\hline 22477-02AR & 16.7 & $0.21 \pm 0.05$ & $7.15 \pm 0.21$ & $91.88 \pm 0.40$ & $103.25 \pm 2.29$ & $96.43 \pm 1.62$ & $2035 \pm 22$ & 132.63 \\
\hline 22477-02AS & 17 & $0.21 \pm 0.05$ & $6.46 \pm 0.19$ & $83.79 \pm 0.45$ & $94.79 \pm 2.16$ & $88.20 \pm 1.53$ & $2039 \pm 22$ & 133.06 \\
\hline $22477-02 \mathrm{AT}$ & 17.3 & $0.15 \pm 0.05$ & $6.42 \pm 0.18$ & $79.05 \pm 0.40$ & $89.42 \pm 2.28$ & $84.57 \pm 1.62$ & $2059 \pm 25$ & 135.25 \\
\hline 22477-02AU & 17.7 & $0.18 \pm 0.05$ & $6.02 \pm 0.17$ & $77.37 \pm 0.37$ & $87.77 \pm 2.10$ & $82.10 \pm 1.49$ & $2049 \pm 23$ & 134.15 \\
\hline 22477-02AV & 18 & $0.14 \pm 0.05$ & $7.02 \pm 0.19$ & $90.31 \pm 0.42$ & $101.93 \pm 2.35$ & $97.44 \pm 1.66$ & $2069 \pm 22$ & 136.36 \\
\hline 22477-02AW & 18.3 & $0.15 \pm 0.05$ & $6.27 \pm 0.18$ & $77.49 \pm 0.42$ & $88.39 \pm 2.27$ & $83.63 \pm 1.61$ & $2070 \pm 25$ & 136.47 \\
\hline 22477-02AX & 18.7 & $0.24 \pm 0.05$ & $6.82 \pm 0.18$ & $87.40 \pm 0.37$ & $99.48 \pm 2.24$ & $91.72 \pm 1.59$ & $2035 \pm 22$ & 132.63 \\
\hline 22477-02AY & 19 & $0.20 \pm 0.05$ & $7.90 \pm 0.19$ & $100.60 \pm 0.45$ & $114.71 \pm 2.33$ & $108.30 \pm 1.65$ & $2067 \pm 20$ & 136.14 \\
\hline $22477-02 \mathrm{AZ}$ & 19.3 & $0.24 \pm 0.05$ & $7.76 \pm 0.19$ & $96.35 \pm 0.41$ & $111.22 \pm 2.25$ & $103.60 \pm 1.60$ & $2065 \pm 20$ & 135.91 \\
\hline 22477-02AAA & 19.7 & $0.29 \pm 0.05$ & $7.75 \pm 0.19$ & $98.13 \pm 0.44$ & $112.46 \pm 2.18$ & $103.20 \pm 1.55$ & $2038 \pm 20$ & 132.95 \\
\hline 22477-02AAB & 20 & $0.24 \pm 0.05$ & $7.62 \pm 0.19$ & $91.04 \pm 0.41$ & $105.11 \pm 2.22$ & $97.52 \pm 1.57$ & $2060 \pm 21$ & 135.36 \\
\hline
\end{tabular}




\begin{tabular}{|c|c|c|c|c|c|c|c|c|c|}
\hline & 22477-02AAC & 20.4 & $0.29 \pm 0.06$ & $9.12 \pm 0.21$ & $108.90 \pm 0.46$ & $124.80 \pm 2.51$ & $115.50 \pm 1.78$ & $2049 \pm 20$ & 134.15 \\
\hline & 22477-02AAD & 20.7 & $0.44 \pm 0.05$ & $10.22 \pm 0.21$ & $119.50 \pm 0.49$ & $138.27 \pm 2.42$ & $124.30 \pm 1.71$ & $2024 \pm 18$ & 131.43 \\
\hline & 22477-02AAE & 21 & $0.29 \pm 0.05$ & $8.59 \pm 0.21$ & $100.20 \pm 0.39$ & $116.48 \pm 2.45$ & $107.10 \pm 1.74$ & $2058 \pm 21$ & 135.14 \\
\hline & 22477-02AAF & 21.4 & $0.30 \pm 0.05$ & $9.11 \pm 0.21$ & $106.30 \pm 0.40$ & $125.24 \pm 2.40$ & $115.60 \pm 1.70$ & $2080 \pm 19$ & 137.58 \\
\hline & 22477-02AAG & 21.7 & $0.24 \pm 0.05$ & $7.32 \pm 0.19$ & $90.24 \pm 0.37$ & $105.26 \pm 2.21$ & $97.60 \pm 1.56$ & $2072 \pm 21$ & 136.69 \\
\hline & 22477-02AAH & 22 & $0.16 \pm 0.05$ & $7.45 \pm 0.19$ & $98.21 \pm 1.62$ & $111.68 \pm 2.26$ & $106.50 \pm 1.60$ & $2076 \pm 28$ & 137.13 \\
\hline & 22477-02AAI & 22.4 & $0.15 \pm 0.05$ & $6.13 \pm 0.18$ & $75.70 \pm 1.68$ & $86.98 \pm 2.12$ & $82.03 \pm 1.51$ & $2075 \pm 36$ & 137.02 \\
\hline & 22477-02AAJ & 22.7 & $0.18 \pm 0.05$ & $6.56 \pm 0.18$ & $83.05 \pm 0.39$ & $95.27 \pm 2.16$ & $89.38 \pm 1.53$ & $2066 \pm 22$ & 136.02 \\
\hline & 22477-02AAK & 23 & $0.15 \pm 0.05$ & $6.32 \pm 0.20$ & $82.32 \pm 0.74$ & $95.98 \pm 2.34$ & $91.28 \pm 1.66$ & $2103 \pm 26$ & 140.16 \\
\hline & 22477-02AAL & 23.4 & $0.18 \pm 0.05$ & $5.01 \pm 0.18$ & $64.58 \pm 0.37$ & $75.99 \pm 2.20$ & $70.28 \pm 1.56$ & $2080 \pm 28$ & 137.58 \\
\hline & 22477-02AAM & 23.7 & $0.12 \pm 0.05$ & $4.07 \pm 0.16$ & $51.56 \pm 0.30$ & $59.02 \pm 2.14$ & $55.17 \pm 1.52$ & $2059 \pm 35$ & 135.25 \\
\hline & 22477-02AAN & 24 & $0.11 \pm 0.04$ & $2.51 \pm 0.15$ & $31.97 \pm 0.30$ & $36.73 \pm 1.99$ & $33.05 \pm 1.41$ & $2017 \pm 53$ & 130.68 \\
\hline & 22477-02AAO & 24.4 & $0.11 \pm 0.05$ & $2.19 \pm 0.16$ & $28.99 \pm 0.26$ & $33.27 \pm 2.17$ & $29.77 \pm 1.53$ & $2009 \pm 64$ & 129.82 \\
\hline & 22477-02AAP & 24.7 & $0.09 \pm 0.04$ & $2.25 \pm 0.15$ & $28.11 \pm 0.29$ & $31.98 \pm 1.99$ & $29.25 \pm 1.40$ & $2025 \pm 60$ & 131.54 \\
\hline & 22477-02AAQ & 25.4 & $0.01 \pm 0.04$ & $1.09 \pm 0.14$ & $14.76 \pm 0.24$ & $17.21 \pm 1.88$ & $17.00 \pm 1.33$ & $2150 \pm 100$ & 145.53 \\
\hline & 22477-02AAR & 26 & & $1.00 \pm 0.13$ & $12.70 \pm 0.22$ & $15.22 \pm 1.87$ & $16.65 \pm 1.33$ & $2320 \pm 110$ & 166.19 \\
\hline & 22477-02AAS & 27 & $0.03 \pm 0.04$ & $1.35 \pm 0.13$ & $18.04 \pm 0.25$ & $21.07 \pm 1.79$ & $20.09 \pm 1.27$ & $2109 \pm 80$ & 140.84 \\
\hline & 22477-02AAT & 35 & $0.12 \pm 0.04$ & $3.12 \pm 0.15$ & $40.65 \pm 0.30$ & $47.23 \pm 1.94$ & $43.29 \pm 1.37$ & $2053 \pm 40$ & 134.59 \\
\hline & 22477-02AAU & 45 & $0.07 \pm 0.04$ & $0.53 \pm 0.13$ & $7.03 \pm 0.22$ & $8.47 \pm 1.87$ & $6.13 \pm 1.32$ & $1820 \pm 250$ & 110.59 \\
\hline B15-053a_03 & $22477-03 \mathrm{~A}$ & 3 & & & $0.00 \pm 0.00$ & $0.00 \pm 0.23$ & $0.21 \pm 0.16$ & $10000 \pm 1600$ & 16150 \\
\hline$J=1.99143 \mathrm{e}-2$ & 22477-03B & 5 & & & & $0.00 \pm 0.21$ & $0.38 \pm 0.15$ & N/A & \\
\hline \multirow[t]{7}{*}{ $\pm 2.87347 \mathrm{e}-5$} & $22477-03 \mathrm{C}$ & 6 & & & & $0.00 \pm 0.22$ & $0.07 \pm 0.15$ & N/A & \\
\hline & 22477-03D & 6.5 & & & & $0.00 \pm 0.21$ & $0.17 \pm 0.15$ & N/A & \\
\hline & $22477-03 E$ & 7 & $0.09 \pm 0.05$ & & & $0.00 \pm 0.17$ & $-0.23 \pm 0.12$ & $12300 \pm 2600$ & 57954 \\
\hline & $22477-03 \mathrm{~F}$ & 9 & $0.20 \pm 0.05$ & & $0.00 \pm 0.00$ & $0.00 \pm 0.17$ & $-0.49 \pm 0.12$ & N/A & \\
\hline & $22477-03 \mathrm{G}$ & 10 & $0.07 \pm 0.05$ & & $0.00 \pm 0.00$ & $0.00 \pm 0.17$ & $-0.17 \pm 0.12$ & N/A & \\
\hline & $22477-03 \mathrm{H}$ & 4 & $0.08 \pm 0.05$ & & $0.00 \pm 0.00$ & $0.00 \pm 0.17$ & $-0.21 \pm 0.12$ & N/A & \\
\hline & 22477-03I & 7 & $0.13 \pm 0.05$ & $0.44 \pm 0.02$ & $11.15 \pm 2.12$ & $12.23 \pm 0.19$ & $11.91 \pm 0.14$ & $2060 \pm 230$ & 135.36 \\
\hline
\end{tabular}




\begin{tabular}{|c|c|c|c|c|c|c|c|c|c|c|}
\hline & $22477-03 \mathrm{~J}$ & 9 & $0.08 \pm 0.06$ & $1.34 \pm 0.04$ & $48.36 \pm 1.73$ & $50.90 \pm 0.23$ & $50.69 \pm 0.17$ & $2033 \pm 44$ & 132.41 & $\mathrm{x}$ \\
\hline & $22477-03 \mathrm{~K}$ & 9.5 & $0.06 \pm 0.06$ & $0.81 \pm 0.06$ & $65.78 \pm 3.08$ & $70.84 \pm 0.25$ & $70.70 \pm 0.19$ & $2064 \pm 58$ & 135.80 & $\mathrm{x}$ \\
\hline & $22477-03 \mathrm{~L}$ & 11 & $0.07 \pm 0.10$ & $12.39 \pm 0.12$ & $190.47 \pm 4.33$ & $204.80 \pm 0.35$ & $204.62 \pm 0.25$ & $2064 \pm 28$ & 135.80 & $\mathrm{x}$ \\
\hline & $22477-03 \mathrm{M}$ & 11.5 & & $11.55 \pm 0.11$ & $180.17 \pm 2.60$ & $192.99 \pm 0.41$ & $193.01 \pm 0.25$ & $2060 \pm 18$ & 135.36 & $\mathrm{x}$ \\
\hline & $22477-03 \mathrm{~N}$ & 13 & $0.22 \pm 0.11$ & $17.28 \pm 0.19$ & $289.48 \pm 3.37$ & $311.75 \pm 0.42$ & $311.19 \pm 0.30$ & $2064 \pm 14$ & 135.80 & $\mathrm{x}$ \\
\hline & $22477-030$ & 14.2 & $0.08 \pm 0.11$ & $18.64 \pm 0.11$ & $282.21 \pm 3.18$ & $302.35 \pm 0.41$ & $302.14 \pm 0.31$ & $2059 \pm 14$ & 135.25 & $\mathrm{x}$ \\
\hline & $22477-03 P$ & 15.4 & $0.11 \pm 0.10$ & $18.30 \pm 0.12$ & $285.00 \pm 4.62$ & $303.78 \pm 0.37$ & $303.50 \pm 0.27$ & $2053 \pm 20$ & 134.59 & $\mathrm{x}$ \\
\hline & 22477-03Q & 16.6 & $0.13 \pm 0.10$ & $17.75 \pm 0.16$ & $299.87 \pm 3.37$ & $320.16 \pm 0.36$ & $319.84 \pm 0.26$ & $2055 \pm 14$ & 134.81 & $\mathrm{x}$ \\
\hline & $22477-03 R$ & 18.1 & $0.16 \pm 0.12$ & $23.54 \pm 0.17$ & $371.08 \pm 2.32$ & $396.16 \pm 0.46$ & $395.76 \pm 0.35$ & $2054.6 \pm 7.8$ & 134.77 & $\mathrm{x}$ \\
\hline & $22477-03 \mathrm{~S}$ & 19.6 & $0.22 \pm 0.13$ & $25.87 \pm 0.22$ & $429.23 \pm 1.85$ & $457.83 \pm 0.51$ & $457.28 \pm 0.39$ & $2053.3 \pm 5.5$ & 134.63 & $\mathrm{x}$ \\
\hline & $22477-03 \mathrm{~T}$ & 21.1 & $0.04 \pm 0.12$ & $23.49 \pm 0.12$ & $331.18 \pm 3.09$ & $353.24 \pm 0.46$ & $353.14 \pm 0.33$ & $2054 \pm 12$ & 134.70 & $\mathrm{x}$ \\
\hline & $22477-03 \mathrm{U}$ & 22.6 & & $14.24 \pm 0.09$ & $200.69 \pm 2.12$ & $213.21 \pm 0.34$ & $213.38 \pm 0.24$ & $2051 \pm 13$ & 134.37 & $\mathrm{x}$ \\
\hline & $22477-03 \mathrm{~V}$ & 25 & & $12.71 \pm 0.12$ & $181.26 \pm 2.22$ & $193.49 \pm 0.29$ & $193.51 \pm 0.23$ & $2056 \pm 15$ & 134.92 & $\mathrm{x}$ \\
\hline & $22477-03 \mathrm{~W}$ & 28 & $0.09 \pm 0.08$ & $7.80 \pm 0.08$ & $108.92 \pm 2.02$ & $115.72 \pm 0.30$ & $115.49 \pm 0.22$ & $2047 \pm 23$ & 133.93 & $\mathrm{x}$ \\
\hline & $22477-03 X$ & 31 & $0.09 \pm 0.10$ & $13.76 \pm 0.09$ & $190.87 \pm 2.89$ & $202.80 \pm 0.37$ & $202.58 \pm 0.27$ & $2049 \pm 19$ & 134.15 & $\mathrm{x}$ \\
\hline & $22477-03 Y$ & 34 & $0.12 \pm 0.06$ & 2.360 .04 & $0.01 \pm 0.00$ & $0.01 \pm 0.22$ & $-0.30 \pm 0.16$ & N/A & & \\
\hline B14-053a_04 & $22477-04 \mathrm{~A}$ & 2.5 & $0.25 \pm 0.07$ & $0.00 \pm 0.00$ & $17.14 \pm 3.08$ & $4.28 \pm 4.74$ & $-7.95 \pm 3.35$ & $\mathrm{~N} / \mathrm{A}$ & & \\
\hline$J=1.99143 \mathrm{e}-2$ & 22477-04B & 4.5 & $1.13 \pm 0.06$ & $0.00 \pm 0.00$ & $76.64 \pm 3.08$ & $49.95 \pm 4.07$ & $-5.36 \pm 2.88$ & N/A & & \\
\hline $\pm 2.87347 \mathrm{e}-5$ & $22477-04 \mathrm{C}$ & 6 & $1.29 \pm 0.10$ & $0.00 \pm 0.00$ & $185.51 \pm 3.28$ & $164.28 \pm 6.58$ & $101.40 \pm 4.65$ & $1329 \pm 47$ & 69.12 & \\
\hline & 22477-04D & 7 & $1.13 \pm 0.08$ & $0.00 \pm 0.00$ & $190.14 \pm 2.99$ & $195.51 \pm 5.34$ & $140.41 \pm 3.78$ & $1632 \pm 34$ & 93.37 & \\
\hline & 22477-04E & 8 & $1.13 \pm 0.09$ & $0.00 \pm 0.00$ & $232.99 \pm 2.99$ & $246.30 \pm 6.05$ & $191.16 \pm 4.28$ & $1747 \pm 29$ & 103.69 & \\
\hline & $22477-04 \mathrm{~F}$ & 9 & $1.03 \pm 0.09$ & $0.00 \pm 0.00$ & $244.89 \pm 3.08$ & $265.46 \pm 6.21$ & $215.18 \pm 4.39$ & $1825 \pm 28$ & 111.08 & \\
\hline & $22477-04 \mathrm{G}$ & 10.2 & $1.26 \pm 0.11$ & $0.00 \pm 0.00$ & $291.58 \pm 2.70$ & $350.30 \pm 7.66$ & $289.00 \pm 5.41$ & $1966 \pm 26$ & 125.27 & \\
\hline & $22477-04 \mathrm{H}$ & 11.4 & $1.57 \pm 0.15$ & $0.00 \pm 0.00$ & $355.43 \pm 5.78$ & $451.49 \pm 10.59$ & $375.09 \pm 7.49$ & $2042 \pm 32$ & 133.39 & $\mathrm{x}$ \\
\hline & 22477-04I & 12.6 & $0.71 \pm 0.12$ & $0.00 \pm 0.00$ & $314.33 \pm 2.99$ & $372.20 \pm 8.14$ & $337.36 \pm 5.76$ & $2063 \pm 25$ & 135.69 & $\mathrm{x}$ \\
\hline & 22477-04J & 13.8 & $0.77 \pm 0.10$ & $19.32 \pm 0.48$ & $273.10 \pm 1.07$ & $332.50 \pm 6.69$ & $295.10 \pm 4.73$ & $2071 \pm 21$ & 136.58 & $\mathrm{x}$ \\
\hline & $22477-04 \mathrm{~K}$ & 15 & $0.44 \pm 0.11$ & $18.45 \pm 0.51$ & $266.80 \pm 4.62$ & $306.97 \pm 7.26$ & $285.40 \pm 5.14$ & $2059 \pm 31$ & 135.25 & $\mathrm{x}$ \\
\hline & $22477-04 \mathrm{~L}$ & 16.2 & $0.48 \pm 0.09$ & $0.00 \pm 0.00$ & $217.08 \pm 1.76$ & $251.27 \pm 5.92$ & $227.88 \pm 4.19$ & $2036 \pm 25$ & 132.73 & $\mathrm{x}$ \\
\hline
\end{tabular}




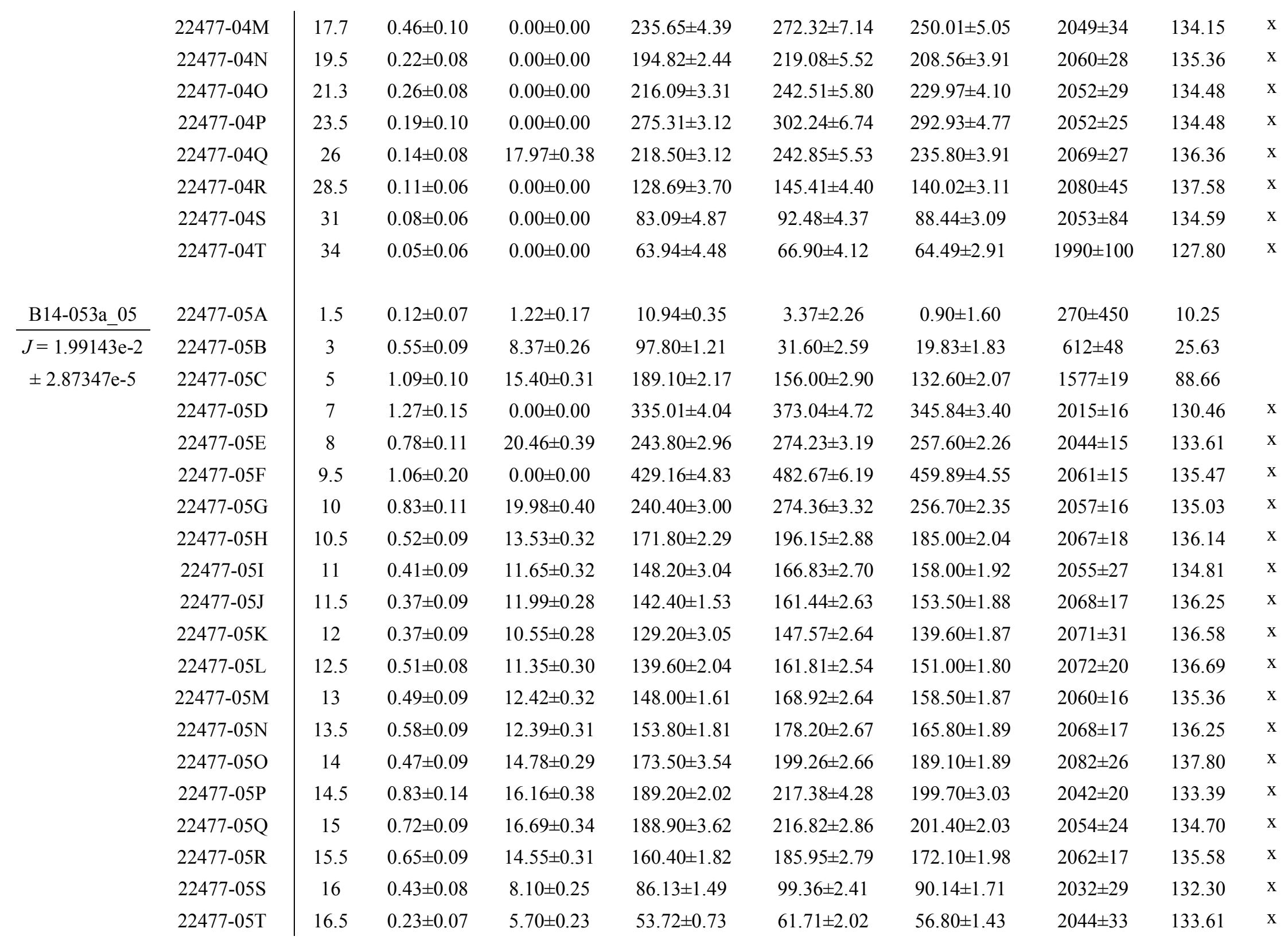




\begin{tabular}{|c|c|c|c|c|c|c|c|c|c|c|}
\hline & $22477-05 \mathrm{U}$ & 18 & $0.12 \pm 0.07$ & $6.86 \pm 0.21$ & $47.36 \pm 0.69$ & $51.63 \pm 2.14$ & $49.02 \pm 1.51$ & $2019 \pm 40$ & 130.89 & $\mathrm{x}$ \\
\hline & $22477-05 \mathrm{~V}$ & 19 & $0.12 \pm 0.07$ & $5.40 \pm 0.20$ & $24.58 \pm 0.81$ & $28.30 \pm 2.11$ & $25.79 \pm 1.49$ & $2035 \pm 81$ & 132.63 & $\mathrm{x}$ \\
\hline & $22477-05 \mathrm{~W}$ & 22 & $0.08 \pm 0.07$ & $8.98 \pm 0.24$ & $20.48 \pm 0.74$ & $23.58 \pm 2.18$ & $21.80 \pm 1.54$ & $2053 \pm 97$ & 134.59 & $\mathrm{x}$ \\
\hline & $22477-05 X$ & 25 & $0.24 \pm 0.07$ & $35.79 \pm 0.38$ & $44.16 \pm 0.95$ & $50.84 \pm 2.07$ & $45.75 \pm 1.46$ & $2020 \pm 46$ & 131.00 & $\mathrm{x}$ \\
\hline & $22477-05 Y$ & 28 & $0.28 \pm 0.07$ & $20.19 \pm 0.30$ & $17.65 \pm 1.19$ & $24.51 \pm 1.97$ & $18.43 \pm 1.40$ & $2030 \pm 120$ & 132.08 & $\mathrm{x}$ \\
\hline & $22477-05 Z$ & 33 & $0.14 \pm 0.06$ & $7.70 \pm 0.22$ & $15.34 \pm 0.60$ & $18.38 \pm 1.91$ & $15.29 \pm 1.35$ & $1970 \pm 116$ & 125.69 & $\mathrm{x}$ \\
\hline B14-080_01 & $22637-01 \mathrm{~A}$ & 3 & $0.71 \pm 0.07$ & $1.05 \pm 0.05$ & $4.77 \pm 2.33$ & $5.19 \pm 0.63$ & $0.88 \pm 0.45$ & $540 \pm 330$ & 22.15 & \\
\hline$J=1.89733 \mathrm{e}-2$ & 22637-01B & 5 & $0.38 \pm 0.09$ & $2.32 \pm 0.07$ & $29.93 \pm 1.22$ & $21.53 \pm 0.81$ & $19.22 \pm 0.57$ & $1437 \pm 50$ & 77.30 & \\
\hline \multirow[t]{12}{*}{ $\pm 6.79989 \mathrm{e}-5$} & $22637-01 \mathrm{C}$ & 7 & $0.20 \pm 0.08$ & $8.44 \pm 0.10$ & $110.92 \pm 2.22$ & $105.89 \pm 0.67$ & $104.69 \pm 0.47$ & $1851 \pm 24$ & 113.61 & \\
\hline & 22637-01D & 9 & $0.00 \pm 0.09$ & $13.51 \pm 0.13$ & $164.24 \pm 1.78$ & $183.02 \pm 0.87$ & $183.00 \pm 0.53$ & $2049 \pm 14$ & 134.15 & $\mathrm{x}$ \\
\hline & $22637-01 \mathrm{E}$ & 11 & $0.20 \pm 0.10$ & $21.62 \pm 0.15$ & $276.83 \pm 2.45$ & $311.73 \pm 0.94$ & $310.54 \pm 0.70$ & $2057 \pm 11$ & 135.03 & $\mathrm{x}$ \\
\hline & $22637-01 \mathrm{~F}$ & 13 & & $16.73 \pm 0.15$ & $208.73 \pm 2.67$ & $232.34 \pm 0.83$ & $232.90 \pm 0.59$ & $2051 \pm 16$ & 134.37 & $\mathrm{x}$ \\
\hline & $22637-01 \mathrm{G}$ & 14.5 & $0.06 \pm 0.07$ & $8.50 \pm 0.10$ & $103.26 \pm 1.22$ & $115.81 \pm 0.60$ & $115.46 \pm 0.42$ & $2053 \pm 15$ & 134.59 & $\mathrm{x}$ \\
\hline & $22637-01 \mathrm{H}$ & 16 & & $5.61 \pm 0.09$ & $67.79 \pm 1.67$ & $75.30 \pm 0.64$ & $76.02 \pm 0.45$ & $2057 \pm 31$ & 135.03 & $\mathrm{x}$ \\
\hline & 22637-01I & 17.5 & $0.16 \pm 0.06$ & $2.02 \pm 0.09$ & $23.98 \pm 1.89$ & $27.56 \pm 0.54$ & $26.56 \pm 0.38$ & $2042 \pm 98$ & 133.39 & $\mathrm{x}$ \\
\hline & 22637-01J & 20.5 & $0.06 \pm 0.08$ & $4.83 \pm 0.08$ & $60.07 \pm 1.67$ & $66.84 \pm 0.67$ & $66.44 \pm 0.47$ & $2040 \pm 35$ & 133.17 & $\mathrm{x}$ \\
\hline & $22637-01 \mathrm{~K}$ & 23.5 & $0.03 \pm 0.08$ & $5.80 \pm 0.09$ & $75.20 \pm 1.34$ & $84.49 \pm 0.71$ & $84.31 \pm 0.51$ & $2057 \pm 23$ & 135.03 & $\mathrm{x}$ \\
\hline & $22637-01 \mathrm{~L}$ & 26.5 & & $1.19 \pm 0.06$ & $15.52 \pm 2.44$ & $17.46 \pm 0.64$ & $17.61 \pm 0.45$ & $2070 \pm 200$ & 136.47 & $\mathrm{x}$ \\
\hline & $22637-01 \mathrm{M}$ & 29.5 & $0.08 \pm 0.08$ & $2.28 \pm 0.07$ & $31.03 \pm 1.78$ & $34.80 \pm 0.65$ & $34.29 \pm 0.46$ & $2039 \pm 72$ & 133.06 & $\mathrm{x}$ \\
\hline & $22637-01 \mathrm{~N}$ & 35 & $0.01 \pm 0.08$ & $2.01 \pm 0.06$ & $27.83 \pm 2.45$ & $29.90 \pm 0.67$ & $29.82 \pm 0.47$ & $2002 \pm 108$ & 129.07 & $\mathrm{x}$ \\
\hline B14-080_02 & $22637-08 \mathrm{~A}$ & 1.5 & $0.53 \pm 0.09$ & $0.92 \pm 0.11$ & $5.97 \pm 0.47$ & $2.03 \pm 0.52$ & $-0.20 \pm 0.37$ & N/A & & \\
\hline$J=1.89733 \mathrm{e}-2$ & 22637-08B & 3 & $0.25 \pm 0.12$ & $0.51 \pm 0.10$ & $5.22 \pm 0.84$ & $2.64 \pm 0.70$ & $1.57 \pm 0.49$ & $820 \pm 230$ & 36.52 & \\
\hline \multirow[t]{5}{*}{ $\pm 6.79989 \mathrm{e}-5$} & $22637-08 \mathrm{C}$ & 5 & $0.34 \pm 0.11$ & $1.62 \pm 0.11$ & $13.98 \pm 0.63$ & $11.14 \pm 0.64$ & $9.69 \pm 0.46$ & $1515 \pm 67$ & 83.52 & \\
\hline & $22637-08 \mathrm{D}$ & 7 & $0.27 \pm 0.07$ & $4.41 \pm 0.16$ & $45.30 \pm 0.50$ & $43.83 \pm 0.42$ & $42.71 \pm 0.30$ & $1850 \pm 15$ & 113.51 & \\
\hline & $22637-08 \mathrm{E}$ & 8 & $0.19 \pm 0.07$ & $4.96 \pm 0.17$ & $52.96 \pm 0.45$ & $55.60 \pm 0.40$ & $54.80 \pm 0.28$ & $1960 \pm 12$ & 124.64 & \\
\hline & $22637-08 \mathrm{~F}$ & 9 & $0.04 \pm 0.07$ & $6.10 \pm 0.16$ & $60.01 \pm 0.55$ & $63.62 \pm 0.45$ & $63.45 \pm 0.33$ & $1986 \pm 12$ & 127.37 & \\
\hline & $22637-08 \mathrm{G}$ & 10 & & $7.43 \pm 0.22$ & $74.91 \pm 0.62$ & $81.01 \pm 0.67$ & $81.30 \pm 0.48$ & $2017 \pm 12$ & 130.68 & \\
\hline
\end{tabular}




\begin{tabular}{|c|c|c|c|c|c|c|c|c|c|c|}
\hline & $22637-08 \mathrm{H}$ & 11 & $0.12 \pm 0.08$ & $10.85 \pm 0.21$ & $112.00 \pm 0.71$ & $125.42 \pm 0.51$ & $124.90 \pm 0.37$ & $2050 \pm 8.1$ & 134.26 & $\mathrm{x}$ \\
\hline & 22637-08I & 12 & $0.27 \pm 0.12$ & $14.56 \pm 0.34$ & $152.20 \pm 3.11$ & $171.75 \pm 0.97$ & $170.60 \pm 0.82$ & $2057 \pm 26$ & 135.03 & $\mathrm{x}$ \\
\hline & $22637-08 \mathrm{~J}$ & 12.5 & & $9.60 \pm 0.19$ & $100.10 \pm 1.38$ & $111.33 \pm 0.74$ & $111.90 \pm 0.55$ & $2053 \pm 18$ & 134.59 & $\mathrm{x}$ \\
\hline & $22637-08 \mathrm{~K}$ & 13 & $0.09 \pm 0.08$ & $6.29 \pm 0.19$ & $68.58 \pm 1.04$ & $76.97 \pm 0.48$ & $76.59 \pm 0.36$ & $2052 \pm 19$ & 134.48 & $\mathrm{x}$ \\
\hline & $22637-08 \mathrm{~L}$ & 13.5 & $0.14 \pm 0.07$ & $4.22 \pm 0.17$ & $42.85 \pm 0.48$ & $48.36 \pm 0.46$ & $47.77 \pm 0.34$ & $2050 \pm 16$ & 134.26 & $\mathrm{x}$ \\
\hline & $22637-08 \mathrm{M}$ & 14 & $0.11 \pm 0.07$ & $3.36 \pm 0.13$ & $36.61 \pm 0.45$ & $41.54 \pm 0.46$ & $41.06 \pm 0.34$ & $2057 \pm 18$ & 135.03 & $\mathrm{x}$ \\
\hline & $22637-08 \mathrm{~N}$ & 15 & $0.11 \pm 0.07$ & $3.04 \pm 0.14$ & $35.90 \pm 0.53$ & $40.69 \pm 0.40$ & $40.22 \pm 0.29$ & $2056 \pm 20$ & 134.92 & $\mathrm{x}$ \\
\hline & $22637-080$ & 17 & $0.07 \pm 0.07$ & $3.90 \pm 0.16$ & $38.94 \pm 0.52$ & $43.90 \pm 0.47$ & $43.61 \pm 0.37$ & $2056 \pm 19$ & 134.92 & $\mathrm{x}$ \\
\hline & $22637-08 \mathrm{P}$ & 19 & $0.02 \pm 0.07$ & $4.71 \pm 0.19$ & $51.04 \pm 0.95$ & $57.24 \pm 0.45$ & $57.14 \pm 0.32$ & $2055 \pm 24$ & 134.81 & $\mathrm{x}$ \\
\hline & 22637-08Q & 21 & & $1.86 \pm 0.12$ & $19.77 \pm 1.30$ & $23.12 \pm 1.08$ & $23.18 \pm 1.04$ & $2112 \pm 99$ & 141.18 & $\mathrm{x}$ \\
\hline & $22637-08 \mathrm{R}$ & 24 & $0.04 \pm 0.10$ & $6.09 \pm 0.17$ & $67.69 \pm 0.63$ & $75.48 \pm 0.65$ & $75.33 \pm 0.47$ & $2048 \pm 13$ & 134.04 & $\mathrm{x}$ \\
\hline & $22637-08 \mathrm{~S}$ & 28 & $0.02 \pm 0.10$ & $4.35 \pm 0.15$ & $46.96 \pm 0.87$ & $52.73 \pm 0.62$ & $52.65 \pm 0.45$ & $2057 \pm 25$ & 135.03 & $\mathrm{x}$ \\
\hline & $22637-08 \mathrm{~T}$ & 33 & $0.08 \pm 0.07$ & $0.61 \pm 0.09$ & $6.83 \pm 0.55$ & $7.53 \pm 0.39$ & $7.18 \pm 0.27$ & $1980 \pm 110$ & 126.74 & $\mathrm{x}$ \\
\hline B14-086_01 & $22638-01 \mathrm{~A}$ & 3 & $68.76 \pm 0.28$ & $11.48 \pm 0.12$ & $9.62 \pm 1.62$ & $216.75 \pm 2.44$ & $-120.23 \pm 2.03$ & $\mathrm{NaN}$ & & \\
\hline$J=1.89937 \mathrm{e}-2$ & 22638-01B & 4 & $8.76 \pm 0.09$ & $1.95 \pm 0.05$ & $3.41 \pm 1.72$ & $31.20 \pm 0.68$ & $-11.72 \pm 0.51$ & $\mathrm{NaN}$ & & \\
\hline $\pm 7.62159 \mathrm{e}-5$ & $22638-01 C$ & 5 & $20.98 \pm 0.20$ & $6.13 \pm 0.09$ & $23.47 \pm 1.76$ & $73.08 \pm 1.47$ & $-29.75 \pm 1.11$ & $\mathrm{NaN}$ & & \\
\hline & 22638-01D & 6 & $3.72 \pm 0.10$ & $5.18 \pm 0.07$ & $27.70 \pm 1.67$ & $39.06 \pm 0.78$ & $20.81 \pm 0.59$ & $1599 \pm 72$ & 90.53 & \\
\hline & $22638-01 \mathrm{E}$ & 7 & $2.75 \pm 0.07$ & $6.69 \pm 0.10$ & & $75.67 \pm 0.74$ & $62.21 \pm 0.65$ & $\mathrm{NaN}$ & & \\
\hline & $22638-01 \mathrm{~F}$ & 8 & $1.13 \pm 0.09$ & $6.64 \pm 0.10$ & $105.40 \pm 2.50$ & $107.35 \pm 0.95$ & $101.80 \pm 0.85$ & $1879 \pm 26$ & 116.38 & \\
\hline & $22638-01 \mathrm{G}$ & 9 & $0.42 \pm 0.08$ & $7.34 \pm 0.09$ & $113.36 \pm 1.86$ & $128.34 \pm 1.03$ & $126.29 \pm 0.95$ & $2050 \pm 17$ & 134.26 & $\mathrm{x}$ \\
\hline & $22638-01 \mathrm{H}$ & 10 & $0.67 \pm 0.08$ & $9.87 \pm 0.12$ & $157.66 \pm 2.42$ & $178.89 \pm 1.31$ & $175.62 \pm 1.25$ & $2050 \pm 15$ & 134.26 & $\mathrm{x}$ \\
\hline & 22638-01I & 11 & $0.25 \pm 0.09$ & $13.47 \pm 0.15$ & $216.20 \pm 4.54$ & $241.84 \pm 1.72$ & $240.60 \pm 1.67$ & $2049 \pm 23$ & 134.15 & $\mathrm{x}$ \\
\hline & $22638-01 \mathrm{~J}$ & 12 & $0.05 \pm 0.12$ & $15.20 \pm 0.13$ & $260.44 \pm 4.84$ & $291.67 \pm 2.07$ & $291.44 \pm 1.99$ & $2056 \pm 19$ & 134.92 & $\mathrm{x}$ \\
\hline & $22638-01 \mathrm{~K}$ & 13 & & $15.47 \pm 0.14$ & $267.71 \pm 5.20$ & $299.27 \pm 2.10$ & $299.36 \pm 2.02$ & $2055 \pm 21$ & 134.81 & $\mathrm{x}$ \\
\hline & 22638-01L & 14 & $0.74 \pm 0.10$ & $14.52 \pm 0.16$ & $249.68 \pm 4.51$ & $280.91 \pm 1.96$ & $277.31 \pm 1.90$ & $2047 \pm 19$ & 133.93 & $\mathrm{x}$ \\
\hline & $22638-01 \mathrm{M}$ & 15.5 & $0.35 \pm 0.13$ & $20.36 \pm 0.19$ & $344.70 \pm 6.12$ & $384.60 \pm 2.64$ & $382.90 \pm 2.56$ & $2047 \pm 18$ & 133.93 & $\mathrm{x}$ \\
\hline & $22638-01 \mathrm{~N}$ & 16.8 & $0.18 \pm 0.13$ & $23.04 \pm 0.18$ & $373.27 \pm 5.76$ & $419.78 \pm 2.84$ & $418.90 \pm 2.77$ & $2059 \pm 15$ & 135.25 & $\mathrm{x}$ \\
\hline & 22638-01O & 18 & $0.06 \pm 0.17$ & $30.05 \pm 0.23$ & $520.90 \pm 7.80$ & $580.67 \pm 3.93$ & $580.40 \pm 3.84$ & $2050 \pm 14$ & 134.26 & $\mathrm{x}$ \\
\hline
\end{tabular}




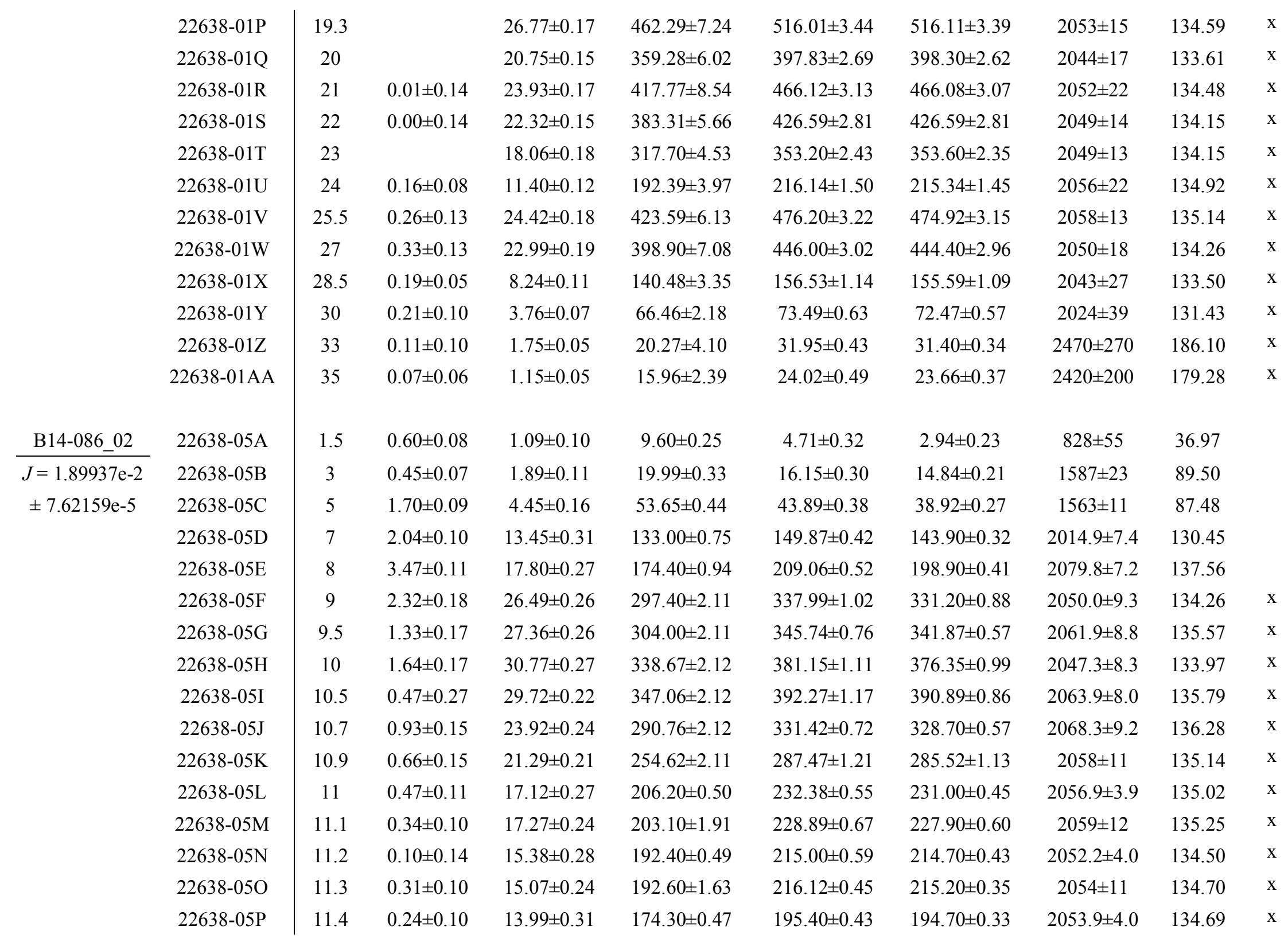




\begin{tabular}{|c|c|c|c|c|c|c|c|c|}
\hline 22638-05Q & 11.5 & $0.30 \pm 0.09$ & $11.38 \pm 0.25$ & $149.80 \pm 0.84$ & $168.38 \pm 0.40$ & $167.50 \pm 0.30$ & $2054.3 \pm 7.3$ & 134.74 \\
\hline $22638-05 R$ & 11.6 & $0.21 \pm 0.09$ & $10.47 \pm 0.25$ & $135.80 \pm 0.73$ & $152.60 \pm 0.40$ & $152.00 \pm 0.29$ & $2056.6 \pm 7.1$ & 134.99 \\
\hline $22638-05 \mathrm{~S}$ & 11.7 & $0.32 \pm 0.08$ & $11.19 \pm 0.27$ & $144.10 \pm 0.42$ & $161.82 \pm 0.38$ & $160.90 \pm 0.29$ & $2053.4 \pm 4.3$ & 134.64 \\
\hline $22638-05 \mathrm{~T}$ & 11.8 & $0.31 \pm 0.08$ & $12.96 \pm 0.26$ & $161.00 \pm 0.84$ & $179.11 \pm 0.40$ & $178.20 \pm 0.32$ & $2042.9 \pm 6.8$ & 133.49 \\
\hline $22638-05 \mathrm{U}$ & 11.9 & $0.27 \pm 0.08$ & $9.97 \pm 0.23$ & $127.30 \pm 0.75$ & $142.91 \pm 0.36$ & $142.10 \pm 0.28$ & $2052.3 \pm 7.7$ & 134.52 \\
\hline $22638-05 \mathrm{~V}$ & 12 & $0.20 \pm 0.08$ & $10.29 \pm 0.24$ & $128.50 \pm 0.73$ & $144.78 \pm 0.39$ & $144.20 \pm 0.32$ & $2059.6 \pm 7.5$ & 135.32 \\
\hline $22638-05 \mathrm{~W}$ & 12.1 & $0.29 \pm 0.08$ & $9.29 \pm 0.21$ & $118.10 \pm 0.72$ & $132.44 \pm 0.42$ & $131.60 \pm 0.34$ & $2050.9 \pm 8.2$ & 134.36 \\
\hline$-05 X$ & 12.2 & $0.20 \pm 0.08$ & $8.98 \pm 0.20$ & $118.20 \pm 0.67$ & $133.00 \pm$ & $.40 \pm 0.29$ & $.5 \pm 7.5$ & 5.09 \\
\hline $22638-05 Y$ & 12.3 & $0.24 \pm 0.08$ & $9.19 \pm 0.24$ & $115.40 \pm 0.64$ & $129.70 \pm 0.36$ & $129.00 \pm 0.26$ & $2054.3 \pm 7.3$ & 134.74 \\
\hline $22638-05 Z$ & 12.4 & $0.30 \pm 0.08$ & $8.43 \pm 0.23$ & $111.30 \pm 1.32$ & $124.99 \pm 0.37$ & $124.10 \pm 0.28$ & $2051 \pm 15$ & 134.37 \\
\hline $22638-05 \mathrm{AA}$ & 12.6 & $0.21 \pm 0.08$ & $9.35 \pm 0.24$ & $122.80 \pm 1.41$ & $138.52 \pm 0.43$ & $137.90 \pm 0.36$ & $2060 \pm 14$ & 135.36 \\
\hline $22638-05 \mathrm{AB}$ & 12.9 & $0.19 \pm 0.08$ & $9.77 \pm 0.22$ & $125.10 \pm 0.73$ & $140.56 \pm 0.36$ & $140.00 \pm 0.27$ & $2055.4 \pm 7.6$ & 134.86 \\
\hline $22638-05 \mathrm{AC}$ & 13.2 & $0.07 \pm 0.09$ & $11.82 \pm 0.25$ & $149.40 \pm 0.46$ & $166.39 \pm 0.46$ & $166.20 \pm 0.38$ & $2048.7 \pm 4.8$ & 134.12 \\
\hline 22638-05AD & 13.5 & $0.16 \pm 0.09$ & $12.62 \pm 0.22$ & $160.10 \pm 0.42$ & $178.88 \pm 0.42$ & $178.40 \pm 0.33$ & $2050.6 \pm 4.0$ & 134.33 \\
\hline $22638-05 \mathrm{AE}$ & 14 & $0.16 \pm 0.10$ & $15.91 \pm 0.26$ & $196.60 \pm 0.85$ & $220.17 \pm 0.46$ & $219.70 \pm 0.35$ & $2054.5 \pm 5.7$ & 134.76 \\
\hline $22638-05 \mathrm{AF}$ & 14.5 & $0.14 \pm 0.10$ & $18.56 \pm 0.31$ & $240.10 \pm 1.75$ & $268.20 \pm 0.48$ & $267.80 \pm 0.37$ & $2051.6 \pm 9.1$ & 134.44 \\
\hline $22638-05 \mathrm{AG}$ & 15 & $0.22 \pm 0.14$ & $21.78 \pm 0.22$ & $278.06 \pm 2.11$ & $314.34 \pm 0.71$ & $313.68 \pm 0.57$ & $2065.8 \pm 9.6$ & 136.00 \\
\hline $22638-05 \mathrm{AH}$ & 15.5 & $0.06 \pm 0.14$ & $20.38 \pm 0.20$ & $270.43 \pm 2.11$ & $303.07 \pm 0.64$ & $302.89 \pm 0.46$ & $2057.0 \pm 9.8$ & 135.03 \\
\hline 22638-05AI & 16 & $0.25 \pm 0.14$ & $20.20 \pm 0.21$ & $261.07 \pm 2.11$ & $297.72 \pm 0.60$ & $297.01 \pm 0.45$ & $2076 \pm 10$ & 137.13 \\
\hline $22638-05 \mathrm{AJ}$ & 16.5 & $0.09 \pm 0.10$ & $17.04 \pm 0.28$ & $217.60 \pm 1.56$ & $245.16 \pm 0.52$ & $244.90 \pm 0.43$ & $2062.9 \pm 9.1$ & 135.68 \\
\hline 22638-05AK & 17 & $0.21 \pm 0.11$ & $17.75 \pm 0.31$ & $233.00 \pm 0.99$ & $260.13 \pm 0.50$ & $259.50 \pm 0.39$ & $2050.2 \pm 5.6$ & 134.29 \\
\hline $22638-05 \mathrm{AL}$ & 17.5 & $0.32 \pm 0.11$ & $18.11 \pm 0.27$ & $237.30 \pm 1.67$ & $266.82 \pm 0.56$ & $265.90 \pm 0.46$ & $2057.4 \pm 8.9$ & 135.08 \\
\hline 22638-05AM & 18 & $0.49 \pm 0.29$ & $20.16 \pm 0.19$ & $264.02 \pm 2.11$ & $298.86 \pm 1.21$ & $297.43 \pm 0.86$ & $2064 \pm 10$ & 135.80 \\
\hline $22638-05 \mathrm{AN}$ & 18.3 & $0.22 \pm 0.15$ & $19.75 \pm 0.19$ & $266.26 \pm 2.11$ & $300.53 \pm 0.64$ & $299.90 \pm 0.48$ & $2064 \pm 10$ & 135.80 \\
\hline $22638-05 \mathrm{AO}$ & 18.6 & $0.30 \pm 0.28$ & $23.58 \pm 0.22$ & $306.88 \pm 2.11$ & $346.53 \pm 1.32$ & $345.67 \pm 1.03$ & $2063.9 \pm 9.3$ & 135.79 \\
\hline 22638-05AP & 19 & $0.25 \pm 0.17$ & $21.35 \pm 0.21$ & $275.15 \pm 2.11$ & $313.84 \pm 1.74$ & $313.12 \pm 1.67$ & $2077 \pm 12$ & 137.24 \\
\hline $22638-05 \mathrm{AQ}$ & 19.2 & & $19.44 \pm 0.21$ & $262.31 \pm 2.11$ & $294.46 \pm 0.62$ & $294.87 \pm 0.47$ & $2061 \pm 10$ & 135.47 \\
\hline 22638-05AR & 19.3 & $0.07 \pm 0.13$ & $20.57 \pm 0.18$ & $270.29 \pm 2.11$ & $302.84 \pm 0.91$ & $302.63 \pm 0.81$ & $2057 \pm 10$ & 135.03 \\
\hline 22638-05AS & 19.4 & $2.11 \pm 0.12$ & $19.55 \pm 0.34$ & $226.30 \pm 1.74$ & $258.68 \pm 0.60$ & $252.50 \pm 0.50$ & $2052.4 \pm 9.8$ & 134.53 \\
\hline
\end{tabular}




\begin{tabular}{|c|c|c|c|c|c|c|c|c|}
\hline 22638-05AT & 19.5 & $0.33 \pm 0.11$ & $18.62 \pm 0.26$ & $246.20 \pm 1.89$ & $276.76 \pm 0.51$ & $275.80 \pm 0.40$ & $2057.0 \pm 9.6$ & 135.03 \\
\hline 22638-05AU & 19.6 & $0.24 \pm 0.09$ & $15.00 \pm 0.23$ & $209.50 \pm 1.75$ & $233.41 \pm 0.63$ & $232.70 \pm 0.57$ & $2047 \pm 11$ & 133.93 \\
\hline 22638-05AV & 19.7 & $0.23 \pm 0.10$ & $12.79 \pm 0.26$ & $174.10 \pm 0.91$ & $194.78 \pm 0.43$ & $194.10 \pm 0.32$ & $2051.5 \pm 6.8$ & 134.43 \\
\hline 22638-05AW & 19.8 & $0.22 \pm 0.10$ & $15.06 \pm 0.28$ & $214.30 \pm 1.66$ & $238.44 \pm 0.58$ & $237.80 \pm 0.51$ & $2045.6 \pm 9.9$ & 133.78 \\
\hline 22638-05AX & 19.9 & $0.23 \pm 0.09$ & $11.31 \pm 0.26$ & $152.50 \pm 0.79$ & $171.87 \pm 0.42$ & $171.20 \pm 0.34$ & $2060.2 \pm 6.9$ & 135.38 \\
\hline 22638-05AY & 20 & $0.12 \pm 0.08$ & $10.40 \pm 0.25$ & $136.40 \pm 0.71$ & $152.16 \pm 0.38$ & $151.80 \pm 0.29$ & $2049.6 \pm 6.8$ & 134.22 \\
\hline 22638-05AZ & 20.1 & & $9.72 \pm 0.22$ & $138.20 \pm 0.78$ & $154.35 \pm 0.51$ & $154.70 \pm 0.37$ & $2056.0 \pm 7.6$ & 134.92 \\
\hline 22638-05BA & 20.2 & $0.16 \pm 0.08$ & $9.24 \pm 0.22$ & $120.60 \pm 0.67$ & .43 & $0 \pm 0.35$ & $5.6 \pm 7.6$ & 3.89 \\
\hline 22638-05BB & 20.3 & $0.07 \pm 0.08$ & $7.24 \pm 0.19$ & $98.00 \pm 0.56$ & $110.00 \pm 0.42$ & $109.80 \pm 0.35$ & $2057.5 \pm 8.1$ & 135.09 \\
\hline $22638-05 B C$ & 20.6 & $0.23 \pm 0.08$ & $9.64 \pm 0.24$ & $127.60 \pm 0.70$ & $143.27 \pm 0.64$ & $142.60 \pm 0.60$ & $2053.9 \pm 8.5$ & 134.69 \\
\hline 22638-05BD & 20.9 & $0.18 \pm 0.08$ & $8.54 \pm 0.20$ & $117.90 \pm 0.65$ & $132.04 \pm 0.42$ & $131.50 \pm 0.35$ & $2051.5 \pm 7.5$ & 134.43 \\
\hline 22638-05BE & 21.2 & $0.14 \pm 0.07$ & $9.20 \pm 0.21$ & $118.90 \pm 0.62$ & $133.11 \pm 0.39$ & $132.70 \pm 0.32$ & $2052.2 \pm 7.1$ & 134.50 \\
\hline 22638-05BF & 21.7 & $0.21 \pm 0.09$ & $15.18 \pm 0.26$ & $209.60 \pm 0.88$ & $235.13 \pm 0.46$ & $234.50 \pm 0.37$ & $2055.8 \pm 5.6$ & 134.90 \\
\hline 22638-05BG & 22.2 & $0.09 \pm 0.17$ & $20.85 \pm 0.22$ & $300.27 \pm 2.11$ & $338.47 \pm 0.93$ & $338.20 \pm 0.78$ & $2063.9 \pm 9.1$ & 135.79 \\
\hline 22638-05BH & 22.7 & $0.32 \pm 0.10$ & $16.54 \pm 0.28$ & $231.00 \pm 0.96$ & $261.03 \pm 0.50$ & $260.10 \pm 0.40$ & $2063.1 \pm 5.5$ & 135.70 \\
\hline 22638-05BI & 23.2 & $0.24 \pm 0.20$ & $36.50 \pm 0.24$ & $491.07 \pm 2.18$ & $551.30 \pm 0.93$ & $550.58 \pm 0.72$ & $2058.3 \pm 5.7$ & 135.18 \\
\hline 22638-05BJ & 23.7 & & $31.62 \pm 0.24$ & $427.42 \pm 2.17$ & $472.71 \pm 3.16$ & $472.71 \pm 3.16$ & $2042 \pm 10$ & 133.39 \\
\hline 22638-05BK & 24.2 & $0.22 \pm 0.10$ & $16.06 \pm 0.26$ & $223.70 \pm 0.91$ & $250.45 \pm 0.45$ & $249.80 \pm 0.34$ & $2053.6 \pm 5.3$ & 134.66 \\
\hline $22638-05 \mathrm{BL}$ & 24.5 & $0.15 \pm 0.11$ & $15.76 \pm 0.25$ & $220.80 \pm 0.66$ & $246.35 \pm 0.48$ & $245.90 \pm 0.36$ & $2049.8 \pm 3.9$ & 134.24 \\
\hline 22638-05BM & 24.8 & $0.20 \pm 0.15$ & $22.13 \pm 0.17$ & $310.28 \pm 2.18$ & $343.41 \pm 0.82$ & $342.83 \pm 0.68$ & $2040.3 \pm 8.8$ & 133.20 \\
\hline 22638-05BN & 25.1 & $0.16 \pm 0.10$ & $14.54 \pm 0.27$ & $203.30 \pm 1.64$ & $226.98 \pm 0.57$ & $226.50 \pm 0.49$ & $2050 \pm 10$ & 134.26 \\
\hline 22638-05BO & 25.4 & $0.42 \pm 0.12$ & $17.87 \pm 0.25$ & $250.70 \pm 2.05$ & $280.34 \pm 0.57$ & $279.10 \pm 0.44$ & $2049 \pm 10$ & 134.15 \\
\hline 22638-05BP & 25.7 & $0.27 \pm 0.10$ & $6.74 \pm 0.16$ & $94.53 \pm 0.74$ & $105.29 \pm 0.43$ & $104.50 \pm 0.32$ & $2041 \pm 10$ & 133.28 \\
\hline 22638-05BQ & 26 & $0.23 \pm 0.09$ & $5.77 \pm 0.17$ & $73.21 \pm 1.00$ & $81.37 \pm 0.37$ & $80.69 \pm 0.27$ & $2037 \pm 17$ & 132.84 \\
\hline 22638-05BR & 26.3 & $0.13 \pm 0.08$ & $6.95 \pm 0.17$ & $98.16 \pm 0.76$ & $109.07 \pm 0.35$ & $108.70 \pm 0.26$ & $2043.0 \pm 9.9$ & 133.50 \\
\hline 22638-05BS & 27 & $0.12 \pm 0.07$ & $6.36 \pm 0.19$ & $97.41 \pm 0.67$ & $108.15 \pm 0.32$ & $107.80 \pm 0.24$ & $2042.5 \pm 8.8$ & 133.44 \\
\hline 22638-05BT & 28 & $0.32 \pm 0.34$ & $82.49 \pm 0.41$ & $1124.53 \pm 2.76$ & $343.71 \pm 1.16$ & $342.83 \pm 0.68$ & $2057.9 \pm 3.1$ & 135.13 \\
\hline 22638-05BU & 28.1 & $0.24 \pm 0.09$ & $13.58 \pm 0.23$ & $176.00 \pm 0.82$ & $196.10 \pm 0.44$ & $195.40 \pm 0.35$ & $2046.5 \pm 6.0$ & 133.88 \\
\hline 22638-05BV & 28.3 & $0.18 \pm 0.08$ & $6.69 \pm 0.16$ & $92.97 \pm 1.16$ & $104.13 \pm 0.36$ & $103.60 \pm 0.27$ & $2051 \pm 16$ & 134.37 \\
\hline
\end{tabular}




\begin{tabular}{|c|c|c|c|c|c|c|c|c|c|c|}
\hline & 22638-05BW & 28.5 & $0.43 \pm 0.10$ & $7.17 \pm 0.19$ & $98.05 \pm 0.69$ & $110.66 \pm 0.43$ & $109.40 \pm 0.32$ & $2052.3 \pm 9.2$ & 134.52 & $\mathrm{x}$ \\
\hline & 22638-05BX & 28.7 & $0.30 \pm 0.09$ & $5.80 \pm 0.17$ & $77.33 \pm 0.63$ & $86.42 \pm 0.41$ & $85.55 \pm 0.31$ & $2042 \pm 11$ & 133.39 & $\mathrm{x}$ \\
\hline & 22638-05BY & 28.9 & $0.27 \pm 0.09$ & $3.63 \pm 0.16$ & $48.16 \pm 0.52$ & $54.24 \pm 0.39$ & $53.45 \pm 0.28$ & $2046 \pm 15$ & 133.83 & $\mathrm{x}$ \\
\hline & 22638-05BZ & 29.3 & $0.08 \pm 0.07$ & $6.19 \pm 0.16$ & $86.99 \pm 0.58$ & $96.56 \pm 0.31$ & $96.31 \pm 0.23$ & $2042.7 \pm 8.6$ & 133.46 & $\mathrm{x}$ \\
\hline & $22638-05 \mathrm{CA}$ & 29.7 & $0.03 \pm 0.08$ & $5.64 \pm 0.16$ & $77.69 \pm 0.55$ & $85.66 \pm 0.33$ & $85.58 \pm 0.25$ & $2036.7 \pm 9.3$ & 132.81 & \\
\hline & $22638-05 \mathrm{CB}$ & 30.1 & & $6.38 \pm 0.20$ & $88.18 \pm 0.56$ & $98.90 \pm 0.33$ & $98.92 \pm 0.24$ & $2058.9 \pm 8.2$ & 135.24 & \\
\hline & $22638-05 \mathrm{CC}$ & 31 & $0.17 \pm 0.08$ & $9.59 \pm 0.18$ & $128.90 \pm 0.75$ & $143.89 \pm 0.35$ & $143.40 \pm 0.26$ & $2048.6 \pm 7.3$ & 134.11 & \\
\hline & 22638-05CD & 33 & $0.11 \pm 0.07$ & $0.77 \pm 0.09$ & $8.28 \pm 0.42$ & $8.87 \pm 0.28$ & $8.54 \pm 0.20$ & $1957 \pm 67$ & 124.33 & \\
\hline B14-086_03 & 22638-09A & 1.5 & $0.40 \pm 0.07$ & $0.43 \pm 0.09$ & $5.07 \pm 0.39$ & $4.12 \pm 0.45$ & $2.39 \pm 0.32$ & $1150 \pm 130$ & 56.60 & \\
\hline$J=1.89937 \mathrm{e}-2$ & 22638-09B & 3 & $0.17 \pm 0.07$ & $0.98 \pm 0.10$ & $15.32 \pm 0.42$ & $17.61 \pm 0.45$ & $16.88 \pm 0.32$ & $2038 \pm 41$ & 132.95 & $\mathrm{x}$ \\
\hline $\pm 7.62159 \mathrm{e}-5$ & 22638-09C & 5 & $1.24 \pm 0.10$ & $10.10 \pm 0.21$ & $147.20 \pm 1.50$ & $168.13 \pm 0.61$ & $162.70 \pm 0.44$ & $2041 \pm 13$ & 133.28 & $\mathrm{x}$ \\
\hline & 22638-09D & 7 & $0.32 \pm 0.20$ & $29.50 \pm 0.29$ & $494.00 \pm 2.22$ & $552.48 \pm 1.44$ & $551.10 \pm 1.14$ & $2052.1 \pm 6.1$ & 134.49 & $\mathrm{x}$ \\
\hline & 22638-09E & 7.2 & $0.28 \pm 0.11$ & $14.59 \pm 0.27$ & $246.40 \pm 0.94$ & $276.14 \pm 0.69$ & $274.90 \pm 0.51$ & $2052.2 \pm 5.2$ & 134.50 & $\mathrm{x}$ \\
\hline & $22638-09 \mathrm{~F}$ & 7.3 & $0.25 \pm 0.09$ & $9.71 \pm 0.19$ & $156.50 \pm 0.90$ & $176.29 \pm 0.63$ & $175.20 \pm 0.49$ & $2056.8 \pm 7.9$ & 135.01 & $\mathrm{x}$ \\
\hline & $22638-09 \mathrm{G}$ & 7.4 & $0.19 \pm 0.08$ & $5.68 \pm 0.17$ & $101.20 \pm 1.15$ & $113.62 \pm 0.52$ & $112.80 \pm 0.39$ & $2051 \pm 15$ & 134.37 & $\mathrm{x}$ \\
\hline & $22638-09 \mathrm{H}$ & 7.6 & $0.12 \pm 0.08$ & $5.69 \pm 0.19$ & $97.38 \pm 0.73$ & $108.94 \pm 0.53$ & $108.40 \pm 0.39$ & $2050 \pm 10$ & 134.26 & $\mathrm{x}$ \\
\hline & 22638-09I & 7.8 & $0.07 \pm 0.08$ & $5.57 \pm 0.17$ & $99.71 \pm 0.77$ & $111.52 \pm 0.52$ & $111.20 \pm 0.40$ & $2051 \pm 10$ & 134.37 & $\mathrm{x}$ \\
\hline & 22638-09J & 8 & $0.18 \pm 0.08$ & $5.38 \pm 0.18$ & $94.78 \pm 0.72$ & $106.67 \pm 0.51$ & $105.90 \pm 0.37$ & $2054 \pm 10$ & 134.70 & $\mathrm{x}$ \\
\hline & $22638-09 \mathrm{~K}$ & 8.2 & $0.10 \pm 0.08$ & $5.82 \pm 0.17$ & $97.94 \pm 0.64$ & $109.33 \pm 0.57$ & $108.90 \pm 0.44$ & $2047.7 \pm 9.4$ & 134.01 & $\mathrm{x}$ \\
\hline & 22638-09L & 8.4 & $0.13 \pm 0.08$ & $6.49 \pm 0.18$ & $105.90 \pm 0.69$ & $118.98 \pm 0.54$ & $118.40 \pm 0.39$ & $2054.5 \pm 9.0$ & 134.76 & $\mathrm{x}$ \\
\hline & $22638-09 \mathrm{M}$ & 8.6 & $0.10 \pm 0.09$ & $7.09 \pm 0.18$ & $114.20 \pm 0.77$ & $127.25 \pm 0.55$ & $126.80 \pm 0.40$ & $2046.7 \pm 9.1$ & 133.90 & $\mathrm{x}$ \\
\hline & $22638-09 \mathrm{~N}$ & 8.8 & $0.03 \pm 0.09$ & $6.03 \pm 0.16$ & $105.80 \pm 0.74$ & $118.73 \pm 0.54$ & $118.60 \pm 0.39$ & $2058.9 \pm 9.4$ & 135.24 & $\mathrm{x}$ \\
\hline & $22638-090$ & 9 & $0.14 \pm 0.09$ & $7.48 \pm 0.20$ & $122.10 \pm 0.74$ & $137.13 \pm 0.57$ & $136.50 \pm 0.42$ & $2054.9 \pm 8.4$ & 134.80 & $\mathrm{x}$ \\
\hline & $22638-09 \mathrm{P}$ & 9.3 & & $6.24 \pm 0.18$ & $112.50 \pm 0.68$ & $125.94 \pm 0.48$ & $126.20 \pm 0.35$ & $2058.4 \pm 8.2$ & 135.19 & $\mathrm{x}$ \\
\hline & 22638-09Q & 9.6 & $0.06 \pm 0.08$ & $7.78 \pm 0.21$ & $131.20 \pm 0.77$ & $147.06 \pm 0.55$ & $146.80 \pm 0.42$ & $2055.8 \pm 8.0$ & 134.90 & $\mathrm{x}$ \\
\hline & $22638-09 R$ & 9.9 & $0.11 \pm 0.08$ & $7.52 \pm 0.20$ & $128.80 \pm 0.74$ & $145.19 \pm 0.53$ & $144.70 \pm 0.38$ & $2060.3 \pm 7.8$ & 135.40 & $\mathrm{x}$ \\
\hline & $22638-09 S$ & 10.2 & $0.08 \pm 0.09$ & $7.53 \pm 0.19$ & $129.60 \pm 0.71$ & $145.65 \pm 0.55$ & $145.30 \pm 0.40$ & $2057.6 \pm 7.5$ & 135.10 & $\mathrm{x}$ \\
\hline & 22638-09T & 10.5 & $0.06 \pm 0.09$ & $6.72 \pm 0.23$ & $122.90 \pm 0.72$ & $136.97 \pm 0.57$ & $136.70 \pm 0.42$ & $2048.1 \pm 8.1$ & 134.06 & $\mathrm{x}$ \\
\hline
\end{tabular}




\begin{tabular}{|c|c|c|c|c|c|c|c|c|}
\hline $22638-09 \mathrm{U}$ & 10.8 & $0.10 \pm 0.08$ & $7.56 \pm 0.22$ & $123.40 \pm 0.87$ & $138.55 \pm 0.55$ & $138.10 \pm 0.42$ & $2055.7 \pm 9.4$ & 134.89 \\
\hline $22638-09 \mathrm{~V}$ & 11.1 & & $7.19 \pm 0.18$ & $129.40 \pm 0.77$ & $143.87 \pm 0.51$ & $144.00 \pm 0.39$ & $2049.1 \pm 8.0$ & 134.16 \\
\hline $22638-09 \mathrm{~W}$ & 11.4 & $0.06 \pm 0.08$ & $6.82 \pm 0.22$ & $120.40 \pm 1.40$ & $134.58 \pm 0.50$ & $134.30 \pm 0.37$ & $2052 \pm 15$ & 134.48 \\
\hline 22638-09X & 11.7 & $0.05 \pm 0.08$ & $6.44 \pm 0.21$ & $115.20 \pm 0.68$ & $128.43 \pm 0.52$ & $128.20 \pm 0.38$ & $2049.4 \pm 8.1$ & 134.20 \\
\hline $22638-09 Y$ & 12 & & $6.38 \pm 0.26$ & $112.00 \pm 0.66$ & $123.47 \pm 0.39$ & $124.10 \pm 0.28$ & $2044.4 \pm 7.7$ & 133.65 \\
\hline $22638-09 Z$ & 12.3 & $0.11 \pm 0.08$ & $6.01 \pm 0.19$ & $107.90 \pm 0.70$ & $121.06 \pm 0.50$ & $120.60 \pm 0.37$ & $2054.3 \pm 8.9$ & 134.74 \\
\hline 22638-09AA & 12.6 & $0.19 \pm 0.08$ & $7.71 \pm 0.17$ & $132.20 \pm 0.76$ & $148.71 \pm 0.51$ & $147.90 \pm 0.38$ & $2055.3 \pm 7.8$ & 134.84 \\
\hline 22638-09AB & 12.9 & & $6.08 \pm 0.16$ & $99.03 \pm 0.68$ & $111.00 \pm 0.47$ & $111.00 \pm 0.33$ & $2058.2 \pm 9.2$ & 135.16 \\
\hline $22638-09 \mathrm{AC}$ & 13.4 & $0.05 \pm 0.08$ & $7.53 \pm 0.18$ & $135.50 \pm 0.69$ & $150.90 \pm 0.52$ & $150.70 \pm 0.40$ & $2047.9 \pm 7.0$ & 134.03 \\
\hline 22638-09AD & 14 & $0.11 \pm 0.09$ & $12.57 \pm 0.25$ & $211.80 \pm 0.78$ & $237.70 \pm 0.60$ & $237.20 \pm 0.44$ & $2056.5 \pm 5.1$ & 134.98 \\
\hline 22638-09AE & 14.5 & $0.10 \pm 0.09$ & $10.43 \pm 0.21$ & $182.80 \pm 3.08$ & $204.33 \pm 0.68$ & $203.90 \pm 0.56$ & $2052 \pm 21$ & 134.48 \\
\hline 22638-09AF & 15 & $0.09 \pm 0.10$ & $12.06 \pm 0.27$ & $216.00 \pm 1.71$ & $240.58 \pm 0.64$ & $240.20 \pm 0.47$ & $2048.2 \pm 10$ & 134.07 \\
\hline $22638-09 A G$ & 15.5 & $0.09 \pm 0.10$ & $14.04 \pm 0.25$ & $245.10 \pm 3.09$ & $274.30 \pm 0.65$ & $273.90 \pm 0.48$ & $2055 \pm 16$ & 134.81 \\
\hline 22638-09AH & 15.8 & $0.11 \pm 0.09$ & $8.95 \pm 0.22$ & $155.70 \pm 0.78$ & $173.68 \pm 0.56$ & $173.20 \pm 0.41$ & $2049.2 \pm 6.8$ & 134.18 \\
\hline 22638-09AI & 16.1 & $0.18 \pm 0.08$ & $8.46 \pm 0.23$ & $144.90 \pm 0.87$ & $162.91 \pm 0.53$ & $162.10 \pm 0.39$ & $2056.0 \pm 8.0$ & 134.92 \\
\hline 22638-09AJ & 16.4 & $0.16 \pm 0.08$ & $6.10 \pm 0.17$ & $104.50 \pm 1.24$ & $116.60 \pm 0.53$ & $115.90 \pm 0.40$ & $2045.1 \pm 15$ & 133.73 \\
\hline 22638-09AK & 16.7 & $0.00 \pm 0.07$ & $5.36 \pm 0.16$ & $92.43 \pm 0.65$ & $103.01 \pm 0.47$ & $103.00 \pm 0.35$ & $2050.4 \pm 9.6$ & 134.31 \\
\hline 22638-09AL & 17 & & $4.53 \pm 0.17$ & $80.63 \pm 0.61$ & $89.89 \pm 0.47$ & $90.18 \pm 0.34$ & $2055.3 \pm 10$ & 134.84 \\
\hline 22638-09AM & 17.5 & $0.33 \pm 0.08$ & $6.07 \pm 0.17$ & $110.90 \pm 0.67$ & $125.13 \pm 0.54$ & $123.70 \pm 0.41$ & $2051.4 \pm 8.4$ & 134.42 \\
\hline 22638-09AN & 18 & & $8.35 \pm 0.21$ & $146.70 \pm 0.82$ & $164.22 \pm 0.58$ & $164.30 \pm 0.44$ & $2057.1 \pm 7.6$ & 135.04 \\
\hline 22638-09AO & 18.5 & $0.59 \pm 0.10$ & $10.49 \pm 0.25$ & $181.20 \pm 1.86$ & $204.88 \pm 0.72$ & $202.30 \pm 0.58$ & $2053 \pm 13$ & 134.59 \\
\hline 22638-09AP & 19 & $0.22 \pm 0.08$ & $8.94 \pm 0.20$ & $153.40 \pm 0.78$ & $172.85 \pm 0.60$ & $171.90 \pm 0.48$ & $2057.6 \pm 7.1$ & 135.10 \\
\hline 22638-09AQ & 19.5 & $0.21 \pm 0.09$ & $9.35 \pm 0.20$ & $159.70 \pm 0.88$ & $178.33 \pm 0.60$ & $177.40 \pm 0.45$ & $2046.6 \pm 7.4$ & 133.89 \\
\hline 22638-09AR & 20 & $0.24 \pm 0.10$ & $8.47 \pm 0.24$ & $152.40 \pm 0.77$ & $170.45 \pm 0.66$ & $169.40 \pm 0.48$ & $2047.7 \pm 7.1$ & 134.01 \\
\hline 22638-09AS & 20.5 & $0.09 \pm 0.10$ & $7.80 \pm 0.21$ & $145.70 \pm 0.94$ & $162.18 \pm 0.69$ & $161.80 \pm 0.52$ & $2046.4 \pm 8.8$ & 133.87 \\
\hline 22638-09AT & 21 & $0.12 \pm 0.10$ & $7.89 \pm 0.23$ & $135.10 \pm 0.78$ & $150.53 \pm 0.61$ & $150.00 \pm 0.45$ & $2045.9 \pm 8.0$ & 133.81 \\
\hline 22638-09AU & 21.5 & $0.15 \pm 0.09$ & $10.94 \pm 0.20$ & $194.50 \pm 1.62$ & $218.35 \pm 0.71$ & $217.70 \pm 0.59$ & $2056 \pm 11$ & 134.92 \\
\hline 22638-09AV & 22 & $0.21 \pm 0.10$ & $11.39 \pm 0.25$ & $202.40 \pm 1.06$ & $227.20 \pm 0.64$ & $226.30 \pm 0.47$ & $2054.5 \pm 6.9$ & 134.76 \\
\hline 22638-09AW & 22.5 & $0.17 \pm 0.10$ & $13.68 \pm 0.28$ & $240.80 \pm 0.89$ & $270.13 \pm 0.77$ & $269.40 \pm 0.64$ & $2055.2 \pm 5.4$ & 134.83 \\
\hline
\end{tabular}




\begin{tabular}{|c|c|c|c|c|c|c|c|c|c|}
\hline 22638-09AX & 23 & $0.32 \pm 0.17$ & $21.99 \pm 0.21$ & $369.58 \pm 2.18$ & $417.45 \pm 1.12$ & $416.03 \pm 0.85$ & $2063.1 \pm 7.7$ & 135.70 & $\mathrm{x}$ \\
\hline 2638-09AY & 23.5 & $0.09 \pm 0.17$ & $27.52 \pm 0.23$ & $483.02 \pm 2.22$ & $538.77 \pm 1.19$ & $538.39 \pm 0.91$ & $2051.0 \pm 6.0$ & 134.37 & $x$ \\
\hline 22638-09AZ & 24 & $0.50 \pm 0.18$ & $24.10 \pm 0.20$ & $418.40 \pm 2.19$ & $466.49 \pm 1.25$ & $464.29 \pm 0.96$ & $2045.6 \pm 6.9$ & 133.78 & $x$ \\
\hline 22638-09BA & 24.3 & $0.23 \pm 0.11$ & $10.46 \pm 0.23$ & $182.80 \pm 0.94$ & $205.22 \pm 0.74$ & $204.20 \pm 0.57$ & $2053.8 \pm 7.2$ & 134.68 & $\mathrm{x}$ \\
\hline 2638-09BВ & 24.6 & $0.05 \pm 0.13$ & $8.30 \pm 0.20$ & $140.60 \pm 0.84$ & $157.00 \pm 0.87$ & $156.80 \pm 0.64$ & $2051.4 \pm 8.9$ & 134.42 & $\mathrm{x}$ \\
\hline 22638-09BC & 24.9 & $0.25 \pm 0.10$ & $7.59 \pm 0.19$ & $131.70 \pm 0.72$ & $147.59 \pm 0.67$ & $146.50 \pm 0.49$ & $2048.4 \pm 7.9$ & 134.09 & $\mathrm{x}$ \\
\hline $2638-09 B D$ & 25.2 & $0.08 \pm 0.11$ & $14.53 \pm 0.22$ & $260.00 \pm 1.25$ & $292.24 \pm 0.75$ & $291.90 \pm 0.57$ & $2059.9 \pm 6.4$ & 135.35 & $x$ \\
\hline 22638-09BE & 25.5 & $0.09 \pm 0.09$ & $10.20 \pm 0.22$ & $176.80 \pm 0.97$ & $198.59 \pm 0.57$ & $198.20 \pm 0.41$ & $2057.8 \pm 7.2$ & 135.12 & $\mathrm{x}$ \\
\hline 22638-09BF & 25.8 & & $6.84 \pm 0.19$ & $126.30 \pm 0.76$ & $139.88 \pm 0.79$ & $140.00 \pm 0.57$ & $2043.9 \pm 8.9$ & 133.60 & $\mathrm{x}$ \\
\hline $2638-09 B G$ & 26.1 & $0.04 \pm 0.08$ & $7.75 \pm 0.20$ & $135.60 \pm 0.70$ & $151.46 \pm 0.50$ & $151.30 \pm 0.37$ & $2052.4 \pm 7.1$ & 134.53 & $\mathrm{x}$ \\
\hline 22638-09BH & 26.5 & $0.18 \pm 0.07$ & $5.08 \pm 0.16$ & $92.00 \pm 1.23$ & $102.81 \pm 0.48$ & $102.00 \pm 0.35$ & $2045 \pm 17$ & 133.72 & $\mathrm{x}$ \\
\hline 22638-09BI & 27 & $0.15 \pm 0.08$ & $6.05 \pm 0.19$ & $101.80 \pm 0.62$ & $113.85 \pm 0.49$ & $113.20 \pm 0.36$ & $2047.8 \pm 8.4$ & 134.02 & $x$ \\
\hline 22638-09BJ & 28 & $0.17 \pm 0.07$ & $3.24 \pm 0.14$ & $49.36 \pm 1.04$ & $55.92 \pm 0.44$ & $55.17 \pm 0.31$ & $2054 \pm 27$ & 134.70 & $\mathrm{x}$ \\
\hline 22638-09BK & 29 & $0.17 \pm 0.07$ & $1.64 \pm 0.12$ & $28.81 \pm 0.81$ & $30.25 \pm 0.43$ & $29.51 \pm 0.31$ & $1949 \pm 36$ & 123.50 & \\
\hline 22638-09BL & 31 & $0.16 \pm 0.07$ & $2.75 \pm 0.14$ & $49.32 \pm 0.60$ & $51.87 \pm 0.45$ & $51.16 \pm 0.33$ & $1964 \pm 16$ & 125.06 & \\
\hline 22638-09BM & 33 & $0.07 \pm 0.07$ & $3.31 \pm 0.13$ & $63.29 \pm 0.54$ & $65.92 \pm 0.49$ & $65.61 \pm 0.37$ & $1963 \pm 12$ & 124.95 & \\
\hline
\end{tabular}

Table DR2. Average Microprobe Analyses of Biotite: $\mathrm{H}_{2} \mathrm{O}$ calculated based on $\mathrm{Cl}+\mathrm{F}+\mathrm{OH}=2.0$ per formula unit.

\begin{tabular}{|c|c|c|c|c|c|c|c|c|c|c|}
\hline & B07-041 & B06-055 & B06-042 & B14-015 & B14-024 & B14-045 & B14-049 & B14-053a & B14-080 & B14-086 \\
\hline $\mathrm{SiO}_{2}$ & 34.23 & 36.18 & 36.93 & 37.09 & 36.34 & 37.50 & 36.92 & 38.16 & 39.52 & 39.87 \\
\hline $\mathrm{TiO}_{2}$ & 4.14 & 4.52 & 4.12 & 3.88 & 3.79 & 3.26 & 3.08 & 3.95 & 2.54 & 3.13 \\
\hline $\mathrm{Al}_{2} \mathrm{O}_{3}$ & 13.70 & 14.11 & 14.34 & 14.06 & 13.94 & 13.73 & 14.00 & 13.46 & 13.86 & 13.81 \\
\hline $\mathrm{MnO}$ & 0.08 & 0.06 & 0.04 & 0.10 & 0.07 & 0.08 & 0.06 & 0.03 & 0.02 & 0.01 \\
\hline $\mathrm{FeO}$ & 27.93 & 21.34 & 18.51 & 13.30 & 16.33 & 15.21 & 15.38 & 11.62 & 8.53 & 6.83 \\
\hline $\mathrm{MgO}$ & 4.82 & 9.86 & 12.31 & 16.89 & 14.52 & 15.44 & 16.28 & 16.88 & 20.16 & 19.98 \\
\hline $\mathrm{K}_{2} \mathrm{O}$ & 9.30 & 9.34 & 9.84 & 7.35 & 8.01 & 9.03 & 7.69 & 9.56 & 9.64 & 9.51 \\
\hline $\mathrm{Na}_{2} \mathrm{O}$ & 0.10 & 0.30 & 0.18 & 0.06 & 0.05 & 0.13 & 0.06 & 0.16 & 0.10 & 0.29 \\
\hline $\mathrm{BaO}$ & 0.24 & 0.26 & 0.23 & 0.09 & 0.07 & 0.13 & 0.09 & 0.20 & 0.21 & 0.17 \\
\hline $\mathrm{CaO}$ & 0.00 & 0.06 & 0.03 & 0.75 & 0.15 & 0.08 & 0.13 & 0.10 & 0.12 & 0.08 \\
\hline $\mathrm{Cl}$ & 0.23 & 0.42 & 0.62 & 0.39 & 0.51 & 0.68 & 0.44 & 0.50 & 0.67 & 0.65 \\
\hline $\mathrm{F}$ & 0.11 & 0.37 & 0.30 & 0.30 & 0.28 & 0.42 & 0.49 & 0.61 & 1.05 & 0.48 \\
\hline $\mathrm{H}_{2} \mathrm{O}$ & 3.59 & 3.62 & 3.68 & 3.77 & 3.66 & 3.61 & 3.62 & 3.61 & 3.46 & 3.75 \\
\hline $\mathrm{O}=\mathrm{F}, \mathrm{Cl}$ & 0.10 & 0.25 & 0.27 & 0.22 & 0.23 & 0.33 & 0.31 & 0.37 & 0.60 & 0.35 \\
\hline
\end{tabular}


\begin{tabular}{ccccccc} 
Total & 98.37 & 100.19 & 100.85 & 98.00 & 97.50 & 98.99 \\
\hline Table DR3. Summary of Isochron Regressions: All uncertainties reported at $1 \sigma$
\end{tabular}

\begin{tabular}{|c|c|c|c|c|c|c|c|c|c|c|c|c|c|}
\hline \multirow{2}{*}{ RunID } & \multicolumn{6}{|c|}{ Isochron } & \multicolumn{6}{|c|}{ Inverse Isochron } & \multirow{2}{*}{ Steps } \\
\hline & Age (Ma) & \pm & MSWD & $\mathrm{p}$ & ${ }^{40} \mathrm{Ar} /{ }^{\beta 6} \mathrm{Ar}$ Int. & \pm & Age (Ma) & \pm & MSWD & $\mathrm{p}$ & ${ }^{40} \mathrm{Ar} /{ }^{\beta 6} \mathrm{Ar}$ Int. & \pm & \\
\hline \multicolumn{14}{|l|}{$\underline{B 07-041}$} \\
\hline $22486-01$ & 2055.8 & 6.2 & 0.10 & 1.00 & 328 & 37 & 2055.8 & 4.0 & 0.28 & 1.00 & 333 & 32 & $\mathrm{D}-\mathrm{W}$ \\
\hline $22486-02$ & 2055.0 & 5.7 & 0.13 & 1.00 & 240 & 190 & 2057.5 & 4.0 & 0.72 & 0.70 & 191 & 63 & $\mathrm{D}-\mathrm{V}$ \\
\hline $22486-03$ & 2057.9 & 9.9 & 0.11 & 1.00 & 140 & 370 & 2054.3 & 7.8 & 0.34 & 0.99 & 360 & 170 & C-R \\
\hline \multicolumn{14}{|l|}{ B06-055 } \\
\hline $22485-01$ & 2055.5 & 3.4 & 0.15 & 1.00 & 301 & 33 & 2055.4 & 3.2 & 0.24 & 1.00 & 302 & 33 & $\mathrm{~A}-\mathrm{O}$ \\
\hline $22485-02$ & 2053.5 & 3.7 & 0.08 & 1.00 & 289 & 31 & 2055.8 & 3.0 & 0.14 & 1.00 & 285 & 28 & B-AK \\
\hline $22485-04$ & 2054.7 & 3.8 & 0.09 & 1.00 & 288 & 20 & 2055.5 & 3.2 & 0.18 & 1.00 & 287 & 19 & B-X \\
\hline \multicolumn{14}{|l|}{$\underline{B 06-042}$} \\
\hline $22484-01$ & 2054.1 & 3.4 & 0.13 & 1.00 & 250 & 110 & 2055.4 & 3.2 & 0.19 & 1.00 & 240 & 90 & $\mathrm{~A}-\mathrm{O}$ \\
\hline $22484-05$ & 2056.0 & 6.4 & 0.08 & 1.00 & 250 & 180 & 2055.7 & 5.1 & 0.15 & 1.00 & 270 & 120 & 04,A-U \\
\hline 22484-06 & 2053.7 & 7.1 & 0.13 & 1.00 & 380 & 430 & 2053.1 & 5.5 & 0.24 & 1.00 & 540 & 250 & D-X \\
\hline \multicolumn{14}{|l|}{ B14-015 } \\
\hline $22487-01$ & 2055.5 & 6.6 & 0.27 & 1.00 & 270 & 140 & 2056.7 & 5.5 & 0.38 & 1.00 & 278 & 85 & F-AB \\
\hline $22487-02$ & 2054 & 14 & 0.16 & 1.00 & 250 & 220 & 2056 & 11 & 0.24 & 0.99 & 240 & 120 & $\mathrm{H}-\mathrm{T}$ \\
\hline $22487-03$ & 2055 & 14 & 0.16 & 1.00 & 2110 & 460 & 2056.6 & 9.9 & 0.35 & 1.00 & 2130 & 350 & $03 \mathrm{~F}-04 \mathrm{~L}$ \\
\hline \multicolumn{14}{|l|}{$\underline{B 14-024}$} \\
\hline $22489-01$ & 2054.4 & 7.1 & 0.07 & 1.00 & 286 & 57 & 2054.7 & 6.5 & 0.08 & 1.00 & 284 & 50 & B-J \\
\hline $22489-02$ & 2048 & 21 & 0.20 & 1.00 & 1160 & 280 & 2053 & 19 & 0.13 & 1.00 & 1200 & 290 & E-V \\
\hline \multicolumn{14}{|l|}{$\underline{B 14-045}$} \\
\hline $22490-02$ & 2058.1 & 4.3 & 0.13 & 1.00 & 340 & 170 & 2054.9 & 4.5 & 0.10 & 1.00 & 450 & 170 & $\mathrm{D}-\mathrm{U}$ \\
\hline $22490-03$ & 2043 & 28 & 0.51 & 0.94 & 2570 & 940 & 1929 & 58 & 0.55 & 0.91 & 7300 & 4900 & F-V \\
\hline $22490-04$ & 2054.5 & 6.5 & 0.09 & 1.00 & 330 & 180 & 2053.6 & 5.9 & 0.17 & 1.00 & 380 & 150 & D-Y \\
\hline \multicolumn{14}{|l|}{ B14-049 } \\
\hline 22488-01 & 2054 & 12 & 0.08 & 1.00 & 290 & 340 & 2054 & 11 & 0.08 & 1.00 & 280 & 270 & C-I \\
\hline
\end{tabular}




\begin{tabular}{|c|c|c|c|c|c|c|c|c|c|c|c|c|c|}
\hline $22488-02$ & 2057.5 & 7.0 & 0.23 & 1.00 & 200 & 170 & 2056.6 & 5.7 & 0.42 & 0.97 & 261 & 99 & $\mathrm{~J}-\mathrm{Z}$ \\
\hline $22488-03$ & 2047 & 12 & 0.37 & 0.98 & 600 & 350 & 2019 & 24 & 0.38 & 0.98 & 1800 & 1100 & $\mathrm{~J}-\mathrm{Y}$ \\
\hline \multicolumn{14}{|l|}{ B14-053a } \\
\hline $22477-02$ & 2042 & 14 & 0.56 & 1.00 & 3200 & 470 & 2042 & 12 & 0.73 & 0.95 & 3670 & 740 & I-AAU \\
\hline $22477-03$ & 2054.2 & 8.0 & 0.09 & 1.00 & 200 & 1300 & 2055.3 & 7.8 & 0.11 & 1.00 & 100 & 91 & $\mathrm{I}-\mathrm{X}$ \\
\hline $22477-04$ & 2054 & 18 & 0.23 & 1.00 & 4860 & 720 & 2057 & 17 & 0.24 & 0.99 & 4880 & 700 & $\mathrm{H}-\mathrm{T}$ \\
\hline $22477-05$ & 2056 & 24 & 0.26 & 1.00 & 2060 & 580 & 2058 & 20 & 0.37 & 0.99 & 2140 & 530 & E-Y \\
\hline \multicolumn{14}{|l|}{ B14-080 } \\
\hline$\overline{22637-01}$ & 2058 & 13 & 0.04 & 1.00 & 300 & 1300 & 2053 & 10 & 0.11 & 1.00 & 630 & 660 & $\mathrm{D}-\mathrm{N}$ \\
\hline $22637-08$ & 2055 & 11 & 0.09 & 1.00 & 300 & 430 & 2051.1 & 9.7 & 0.10 & 1.00 & 460 & 350 & $\mathrm{H}-\mathrm{T}$ \\
\hline \multicolumn{14}{|l|}{ B14-086 } \\
\hline$\overline{22638-01}$ & 2049 & 14 & 0.05 & 1.00 & 460 & 570 & 2053.1 & 7.8 & 0.10 & 1.00 & 380 & 220 & G-Y \\
\hline $22638-05$ & 2051.1 & 5.2 & 0.48 & 1.00 & 336 & 65 & 2054.0 & 5.1 & 0.79 & 0.89 & 294 & 35 & F-BZ \\
\hline $22638-09$ & 2052.8 & 5.3 & 0.20 & 1.00 & 340 & 120 & 2053.6 & 5.2 & 0.31 & 1.00 & 362 & 74 & B-BJ \\
\hline
\end{tabular}


Table DR4. Summary of Ages from Plateau Diagrams: All uncertainties are reported at $1 \sigma$.

\begin{tabular}{ccccccc} 
RunID & Plateau Age (Ma) & \pm & MSWD & $p$ & Integrated Age (Ma) & \pm \\
\hline B07-041 & & & & & & \\
\hline $22486-01$ & 2055.8 & 3.3 & 0.26 & 1.00 & 2047.7 & 6.3 \\
$22486-02$ & 2054.7 & 2.1 & 0.60 & 0.90 & 2050.0 & 3.2 \\
$22486-03$ & 2054.1 & 3.2 & 0.31 & 0.99 & 2036.5 & 4.8
\end{tabular}

$\underline{\mathrm{B} 06-055}$

22485-01

22485-02

22485-04

B06-042

22484-01

22484-05

22484-06

B14-015

22487-01

22487-02

22487-03

B14-024

22489-01

22489-02

B14-045

22490-02

22490-03

22490-04

B14-049

22488-01

22488-02

22488-03

B14-053a

22477-01

22477-02

22477-03

22477-04

22477-05

B14-080

22637-01

22637-08

B14-086

2055.4
2055.8
2055.8

2.1

1.8

0.22

0.14

2.1

0.21

2055.4

2055.7

2053.8

2056.7

2055.2

2056.6

2.0

0.17

3.2

0.14

2.6

0.33

2.0

0.36

0.23

3.3

4.3

0.33

1.00

1.00

1.00

1.00

1.00

1.00

1.00

1.00

1.00

2054.5

4.8

0.05

1.00

0.15

1.00

1.00

2.2

0.28

0.56

0.91

0.15

1.00

2.2

2054.3

$$
5.7
$$

0.06

1.00

0.99

2056.4

3.0

0.38

4.7

0.47

0.95

$\begin{array}{ll}4.3 & 0.59\end{array}$

0.97

3.2

0.72

0.95

1.00

3.2

0.09

8.3

0.21

1.00

0.89

0.65

6.3

0.07

1.00

0.11

1.00

0.37

0.99

2052.4

3.9

.

$\begin{array}{ll}2054.8 & 2.9 \\ 2054.3 & 3.3 \\ 2055.4 & 3.4\end{array}$
3.2 .8 9 


\begin{tabular}{lcccccc}
$22638-05$ & 2053.95 & 0.83 & 0.75 & 0.94 & 2052.7 & 2.0 \\
$22638-09$ & 2053.3 & 1.0 & 0.33 & 1.00 & 2051.6 & 2.3 \\
\hline
\end{tabular}

